\title{
MECÂNICA E GEOMETRIA
}

\author{
Márcio Santos da Rocha
}

\section{DISSERTAÇÃO APRESENTADA}

AO

INSTITUTO DE MATEMÁTICA E ESTATÍSTICA

DA

UNIVERSIDADE DE SÃO PAULO

PARA OBTENÇÃO DO GRAU DE

MESTRE EM

MATEMÁTICA APLICADA

Orientadora: Profa. Dra. Helena Maria Ávila de Castro São Paulo, fevereiro de 1994. 
Aos

meus pais: Antônio e Darcy irmãos: Marco, Márcia e Marta companheira: Nadir. 


\section{Agradecimentos}

Agradeço a todos os colegas que colaboraram com mais esta etapa da minha formação. Existem colegas do Instituto de Matemática e Estatística, bem como colegas da Universidade Estadual de Londrina que merecem um agradecimento especial; não citarei nomes para não ser injusto com outros.

Gostaria de destacar o empenho com o qual a Profa. Dra. Helena Maria Ávila de Castro me orientou; com certeza estarei eternamente agradecido e, sem ela talvez muitos dos obstáculos enfrentados durante o Curso de Mestrado não seriam ultrapassados.

Durante o Curso de Mestrado, recebi apoio financeiro do CNPq - Conselho Nacional de Desenvolvimento Científico e Tecnológico. Certamente, sem tal apoio, não seria possível concluir esse curso; sou imensamente grato.

Deixo aqui um agradecimento especial aos funcionários da biblioteca do Instituto de Matemática e Estatística da USP.

Márcio Santos da Rocha. 


\section{Resumo}

Neste trabalho a Mecânica Clássica é estudada sob o ponto de vista da Geometria Riemanniana. Um sistema mecânico simples é a terna ( $\mathrm{M}, \mathrm{g}, \mathrm{V})$ onde $(\mathrm{M}, \mathrm{g})$, sendo $\mathrm{M}$ o espaço das configurações, é uma variedade Riemanniana, cuja métrica é definida pela energia cinética do sistema, e $\mathrm{V}$ é a energia potencial.

As trajetórias físicas de um sistema mecânico simples conservam a energia total. As trajetórias de energia $h$ são dadas como geodésicas de $\left(M_{h}, g_{h}\right)$, onde $M_{h}$ é o espaço das configurações possíveis, e $\mathrm{g}_{\mathrm{h}}=2(\mathrm{~h}-\mathrm{V}) \mathrm{g}$. As propriedades geométricas desta variedade são estudadas através de suas curvaturas. São dados resultados sobre os sinais das curvaturas nas vizinhanças da fronteira de $M_{h}$ e dos pontos críticos do potencial V.

Exemplos Clássicos da Mecânica Analítica são usados para ilustração, como o Problema de Kepler, o problema de Três Partículas e o Pêndulo Duplo. Um programa em linguagem $\mathrm{C}$ foi usado para obter maiores informações sobre as trajetórias físicas do Pêndulo Duplo. 


\section{Abstract}

Classical Mechanics is studied from the point of view of Riemannian Geometry. A simple mechanical system is the triplet $(\mathrm{M}, \mathrm{g}, \mathrm{V})$ where $(\mathrm{M}, \mathrm{g})(\mathrm{M}$ the configuration space) is a Riemannian Manifold, the metric $\mathrm{g}$ is defined from the kinetic energy of system, and V is the potencial energy. The physical paths of a simple mechanical system conserve the total energy.

The physical paths of energy $h$ are the geodesics of $\left(M_{h}, g_{h}\right)$, where $M_{h}$ is the admissible configuration space, and $\mathrm{g}_{\mathrm{h}}=2(\mathrm{~h}-\mathrm{V}) \mathrm{g}$ is the Jacobi metric. Geometrical properties of the manifold $\left(\mathrm{M}_{\mathrm{h}}, \mathrm{g}_{\mathrm{h}}\right)$ are studied throught its curvatures. Same results on the signal of the curvatures in a neighborhood of the boundary of $\mathrm{M}_{\mathrm{h}}$ and of the critical points of the potential $\mathrm{V}$ are presented.

Classical examples of Analitical Mechanics are presented as illustration, such as the Kepler Problem, the Three Body Problem and the Double Pendulum. A program in language $\mathrm{C}$ is used in order to obtain numerical information about the physical paths of the Double Pendulum. 


\section{Índice}

\section{Capítulo 1}

Introdução

\section{Capítulo 2}

2.0 Introdução 5

2.1 Geometria 6

2.2 Sistema Mecânico Simples 17

2.3 Conexões Projetivamente Equivalentes 23

\section{Capítullo 3}

3.0 Introdução 31

3.1 O tensor Curvatura h-Mecânico $\mathrm{R}^{*}$

3.2 O tensor Ric ${ }^{*}$, Curvatura Escalar Mecânica, e a Curvatura Seccional

Mecânica

3.3 O Sinal da curvatura Mecânica

3.4 Funções Curvatura de um Sistema Mecânico Simples 50

3.5 Curvatura Mecânica em pontos Críticos do potencial V 58 


\section{Capítulo 4}

4.1 O Problema de n-Partículas 61

4.2 O Problema de Força Central 62

4.3 O Problema de Kepler com o Potencial Newtoniano 67

4.4 O Problema de Três Partículas $\quad 70$

4.5 O Pêndulo Duplo 84

Apêndice 116

$\begin{array}{ll}\text { Bibliografia } & 119\end{array}$ 


\section{Capítulo 1}

\section{Introdução}

A Mecânica Clássica tem sido uma das áreas mais importantes da Matemática Aplicada, tendo servido como mola propulsora para o desenvolvimento do cálculo, e se beneficiou ao longo dos séculos com o desenvolvimento da Matemática, como é o caso de suas formulações Lagrangiana e Hamiltoniana. Mais recentemente, a Mecânica inspirou o desenvolvimento da teoria das equações diferenciais, teorias de estabilidade, e até dos Sistemas Dinâmicos, tais como conhecidas atualmente.

Nesta visão mais moderna, a Mecânica absorveu de forma natural a linguagem da Geometria Riemanniana: o espaço de fase de qualquer sistema mecânico com n partículas é uma variedade diferenciável, subvariedade de $\mathrm{R}^{3 \mathrm{n}}$. $\mathrm{O}$ objetivo deste trabalho é descrever em linguagem geométrica as propriedades de um sistema mecânico e através do estudo de propriedades geométricas de variedades convenientes, obter informações sobre a trajetória física deste sistema.

A Mecânica Clássica é descrita, neste contexto, como um sistema mecânico simples (ou, conforme alguns autores, sistema de Jacobi) definido pela terna 
$(\mathrm{M}, \mathrm{g}, \mathrm{V})$, onde $\mathrm{M}$ é uma variedade Riemanniana diferenciável, que é o Espaço de Configuração; g é a métrica Riemanniana em $\mathrm{M}$ associada à função energia cinética $\mathrm{K}$, i.e., $\mathrm{g}(X, X)=2 \mathrm{~K}(X, X), \forall X \in \mathrm{T}_{\mathrm{p}} \mathrm{M}$, e $\mathrm{V}$ é a função energia potencial do sistema.

Para um sistema mecânico simples, a energia total do sistema é conservada numa trajetória física. Assim, para cada valor de energia $h, h$ valor regular de $\mathrm{V}$, define-se uma variedade Riemanniana $\left(\mathrm{M}_{\mathrm{h}}, \mathrm{g}_{\mathrm{h}}\right)$ por $\mathrm{M}_{\mathrm{h}}=\{\mathrm{x} \in \mathrm{M} ; \mathrm{V}(\mathrm{x})<\mathrm{h}\}$ (ou seja, $\mathrm{M}_{\mathrm{h}}$ é subvariedade aberta de $\mathrm{M}$ ) e $\mathrm{g}_{\mathrm{h}}=2(\mathrm{~h}-\mathrm{V}) \mathrm{g}$ (métrica de Jacobi). Esta variedade assim definida é chamada de espaço de h-configuração. Pode-se mostrar que as trajetórias de energia h do sistema mecânico original são, a menos de reparametrização, as geodésicas do espaço das h-configurações. Assim, o estudo das propriedades geométricas de $\left(\mathrm{M}_{\mathrm{h}}, \mathrm{g}_{\mathrm{h}}\right)$ e em particular de suas curvaturas nos dão informações sobre o comportamento do sistema mecânico.

Foi conveniente dividir o texto em quatro capítulos, sendo este primeiro introdutório.

No segundo capítulo, descrevemos as ferramentas geométricas necessárias ao trabalho, e apresentamos a formulação geométrica da Mecânica.

Na seção 1 definem-se conceitos básicos, tais como conexão Riemanniana e suas propriedades, tensores de curvatura e as noções de curvatura de Ricci e 
curvatura escalar. São definidas aplicações especiais, como o gradiente, hessiana e o laplaciano, que generalizam para a geometria, os conceitos clássicos do cálculo. A seção 2, consiste num dos pontos fundamentais do trabalho, pois é nela que é descrita a ligação entre a Geometria Riemanniana e a Mecânica Clássica. São demonstradas as propriedades já citadas de $\left(\mathrm{M}_{\mathrm{h}}, \mathrm{g}_{\mathrm{h}}\right)$ e é estudada a relação entre as conexões Riemanniana de $(\mathbf{M}, \mathrm{g})$ e $\left(\mathrm{M}_{\mathrm{h}}, \mathrm{g}_{\mathrm{h}}\right)$. Na seção 3, encontram-se resultados sobre sistemas mecânicos equivalentes, que são caracterizados por existir um difeomorfismo $\phi$, levando geodésicas sobre geodésicas, a menos de reparametrização.

No capítulo 3, são estudadas as curvaturas do sistema mecânico $\left(\mathrm{M}_{\mathrm{h}}, \mathrm{g}_{\mathrm{h}}, \mathrm{V}\right)$ para obter informações sobre as geodésicas da variedade $\left(M_{h}, g_{h}\right)$. A seção 1 descreve a relação entre o tensor curvatura $R$ da variedade Riemanniana $(M, g)$ e o tensor curvatura $R^{*}$ da variedade Riemanniana $\left(M_{h}, g_{h}\right)$. A seção 2 contém estudos do tensor mecânico Ric ${ }^{*}$, da curvatura escalar mecânica e da curvatura seccional mecânica. Na seção 3 são dados resultados envolvendo os sinais das curvaturas em uma vizinhança do bordo $\partial M_{h}=\{x \in M ; V(x)=h\}$. A seção 4, usa a Álgebra Linear como ferramenta para introduzir funções curvaturas $\mathrm{Q}_{\mathrm{i}}(\mathrm{x}), \mathrm{i}=1, \ldots, \mathrm{m}, \mathrm{m}$ $=\mathrm{C}_{\mathrm{n}, 2}$ e $\mathrm{n}=\operatorname{dimM}$, que serão utilizadas na seção 5 para estudar a curvatura mecânica em uma vizinhança dos pontos críticos do potencial V.

No Capítulo 4 os resultados apresentados são ilustrados através de suas 
aplicações em 5 exemplos: O Problema de n-Partículas, O Problema de Força Central, O Problema de Kepler, O Problema de Três Partículas, O Pêndulo Duplo. Nos problemas de Força Central e de Kepler, são dados resultados envolvendo o sinal da curvatura gaussiana mecânica. No problema de três partículas, apresentamos vários resultados obtidos através da aplicação dos teoremas do capítulo 3. Mostramos também que, contrariando o que está escrito em [8], o potencial $V$ não tem pontos críticos para nenhum valor das massas $m_{1}, m_{2}, m_{3}$ envolvidas, o que implica que as variedades diferenciáveis h-mecânicas estão sempre bem definidas. Para complementar o estudo do Pêndulo Duplo, fizemos um programa em linguagem C, que utiliza o método de Runge-Kutta para calcular numericamente as soluções das equações das geodésicas (este programa está no Apêndice). Os dados são aproveitados pelo simulador algébrico MapleV, onde são esboçados gráficos que ajudam a exemplificar a teoria deste trabalho. Neste exemplo também estão resultados sobre os sinais da curvatura gaussiana mecânica e outros envolvendo o comportamento das geodésicas da variedade Riemanniana $\left(\mathrm{M}_{\mathrm{h}}, \mathrm{g}_{\mathrm{h}}\right)$. As figuras ilustrando o exemplo, caracterizam alguns comportamentos das trajetórias físicas do Pêndulo Duplo. 


\section{Capítullo 2}

\section{Estrutura dos Sistemas Mecânicos}

\section{\# 2.0. Introdução}

O objetivo do capítulo 2, é apresentar alguns conceitos da Geometria Riemanniana e da Mecânica que são usados como base dos capítulos posteriores, bem como descrever a teoria da Mecânica Geométrica.

$\mathrm{Na}$ seção 1, definem-se conceitos básicos, tais como conexão Riemanniana e suas propriedades, tensores de curvatura e as noções de curvatura de Ricci e curvatura escalar. São definidas aplicações especiais, como o gradiente, hessiana e o laplaciano, que generalizam para a geometria, os conceitos clássicos do Cálculo. As noções básicas de Geometria são assumidas conhecidas. As demonstrações das proposições clássicas enunciadas nesta seção se encontram nas referências [1] e [2].

Na seção 2, encontra-se a formulação geométrica da Mecânica. Nesta seção estão as definições de sistema mecânico simples e a de curva física. São demonstradas as propriedades já citadas de $\left(\mathrm{M}_{\mathrm{h}}, \mathrm{g}_{\mathrm{h}}\right)$ e é estudada a relação entre as 
conexões Riemanniana de $(\mathrm{M}, \mathrm{g})$ e $\left(\mathrm{M}_{\mathrm{h}}, \mathrm{g}_{\mathrm{h}}\right)$. Das proposições, destaca-se o teorema de Jacobi por fornecer uma importante relação entre curvas físicas e geodésicas. Ainda nesta seção se encontra o resultado básico que é a conservação da energia ao longo de uma curva física. Esta é a seção que faz o elo entre a Geometria Riemanniana e a Mecânica Clássica.

Na seção 3, encontram-se resultados sobre sistemas mecânicos equivalentes que são caracterizados por existir um difeomorfismo $\phi$, levando geodésicas sobre geodésicas, a menos de reparametrização.

\section{\# 2.1. Geometria}

No transcorrer do trabalho, $\mathrm{M}^{\mathrm{n}}$ denota uma variedade conexa de dimensão n e g uma métrica Riemanniana em $\mathrm{M}^{\mathrm{n}}$. Sempre que houver necessidade de identificar o ponto $\mathrm{p} \in \mathrm{M}$, denotaremos $\mathrm{g}(\mathrm{p})=\langle,\rangle_{\mathrm{p}}$, caso contrário por

$$
\mathrm{g}(\mathrm{X}, \mathrm{Y})=\langle\mathrm{X}, \mathrm{Y}\rangle
$$

O par $\left(\mathrm{M}^{\mathrm{n}}, \mathrm{g}\right)$ denotará uma Variedade Riemanniana conexa de dimensão n. Quando não existir dúvida sobre a métrica e a dimensão, a variedade Riemanniana será representada por $\mathrm{M}$.

Definição 2.1.1. Considere uma aplicação diferenciável 


$$
\mathrm{f}: \mathrm{M}^{\mathrm{m}} \rightarrow \mathrm{N}^{\mathrm{n}}
$$

de uma variedade diferenciável $M$ de dimensão m para uma variedade diferenciável $\mathrm{N}$ de dimensão $\mathrm{n}$. Seja $\mathrm{C}$ o conjunto de todos os pontos, tais que

$$
\mathrm{df}_{\mathrm{p}}: \mathrm{T}_{\mathrm{p}} \mathrm{M} \rightarrow \mathrm{T}_{\mathrm{f}(\mathrm{p})} \mathrm{N}
$$

não é sobrejetora. Então $C$ é chamado o conjunto dos pontos críticos de $f, f(C) o$ conjunto dos valores críticos de $\mathrm{f}$, e o complementar $\mathrm{N}$-f(C) é o conjunto dos valores regulares de $f$.

A proposição abaixo é fundamental para o que segue, e sua demonstração encontra-se em [ 7 ].

Proposição 2.1.2. Seja $\mathrm{f}: \mathrm{M} \rightarrow \mathrm{R}$ uma aplicação diferenciável de uma variedade de dimensão $\mathrm{n}$ a valores reais. Se $0 \in \mathrm{R}$ é um valor regular de $\mathrm{f}$, então o conjunto

$$
N=\{p \in M ; f(p) \leq 0\}
$$

é uma variedade diferenciável de dimensão $\mathrm{m}$, com bordo igual a $\mathrm{f}^{-1}(0)$

A definição de gradiente de uma função em $R^{n}$ pode ser estendida de forma natural a funções sobre variedades da seguinte forma:

Definição 2.1.3. Seja $M$ uma variedade Riemanniana e $f: M \rightarrow R$ uma 
aplicação $\mathbb{C}^{\infty}(\mathrm{M})$. O campo vetorial diferencial gradf $\in \chi(\mathrm{M})$ definido por

$$
\langle\operatorname{grad} f(p), X(p)\rangle_{p}=d f_{p}(x(p))
$$

$\mathrm{p} \in \mathrm{M}$ e $\mathrm{X} \in \chi(\mathrm{M})$ é chamado de gradiente da função $\mathrm{f}$.

Recordamos a seguir as definições de formas diferenciáveis e tensores.

Definição 2.1.4. Seja $T_{p} M^{*}$ o dual do espaço tangente $T_{p} M$. Definimos a k-ésima potência exterior $\Lambda^{\mathrm{k}}\left(\mathrm{T}_{\mathrm{p}} \mathrm{M}^{*}\right)$ como sendo o conjunto das aplicações

$$
\Phi: \mathrm{T}_{\mathrm{p}} \mathrm{M} \underset{\mathrm{k} \text {-vezes }}{\times \ldots} \times \mathrm{T}_{\mathrm{p}} \mathrm{M} \rightarrow \mathrm{R}
$$

tal que

a)(linear) a.1) $\Phi\left(\mathrm{X}_{1}, \ldots, \mathrm{fX}_{\mathrm{i}}, \ldots, \mathrm{X}_{\mathrm{k}}\right)=\mathrm{f} \Phi\left(\mathrm{X}_{1}, \ldots, \mathrm{X}_{\mathrm{i}}, \ldots, \mathrm{X}_{\mathrm{k}}\right), \mathrm{f} \in \mathbb{C}^{\infty}(\mathrm{M})$ e $\mathrm{i}=1, \ldots, \mathrm{n}$

a.2) $\Phi(\ldots, X+Y, \ldots)=\Phi(\ldots, X, \ldots)+\Phi(\ldots, Y, \ldots)$,

b)(alternada) $\Phi\left(\ldots, X_{j}, \ldots, X_{1}, \ldots\right)=-\Phi\left(\ldots, X_{1}, \ldots, X_{j}, \ldots\right)$

Uma forma diferenciável de grau $\mathrm{k}$, ou k-forma, sobre um aberto $\mathrm{U} \subset$ $M$ é uma aplicação $\Phi: p \rightarrow \Phi_{\mathrm{p}} \in \Lambda^{\mathrm{k}}\left(\mathrm{T}_{\mathrm{p}} \mathrm{M}^{*}\right)$ com $\mathrm{p} \in \mathrm{U}$, diferenciável, no sentido que $\mathrm{p} \rightarrow \Phi_{\mathrm{p}}\left(\mathrm{X}_{1}(\mathrm{p}), \ldots, \mathrm{X}_{\mathrm{k}}(\mathrm{p})\right)$ é uma aplicação diferenciável para todo $\mathrm{X}_{1}, \ldots, \mathrm{X}_{\mathrm{k}} \in$ $\chi(\mathrm{M})$.

Definição 2.1.5. Um tensor do tipo $(r, s)$ é uma aplicação

$$
\begin{aligned}
& \mathrm{T}: \chi(\mathrm{M}) \times \ldots \times \chi(\mathrm{M}) \rightarrow \chi(\mathrm{M}) \times \ldots \times \chi(\mathrm{M}) \\
& \text { r-vezes s-vezes }
\end{aligned}
$$


que é linear em cada $\chi(\mathrm{M})$, considerado como um módulo sobre $\mathbb{C}^{\infty}(\mathrm{M})$, isto é,

a) $\quad \mathrm{T}(\ldots, \mathrm{X}+\mathrm{Y}, \ldots)=\mathrm{T}(\ldots, \mathrm{X}, \ldots)+\mathrm{T}(\ldots, \mathrm{Y}, \ldots)$

b) $\quad \mathrm{T}(\ldots, \mathrm{fX}, \ldots)=\mathrm{fT}(\ldots, \mathrm{X}, \ldots)$

$\mathrm{f} \in \mathbb{C}^{\infty}(\mathrm{M}), \mathrm{X}, \mathrm{Y} \in \chi(\mathrm{M})$.

Observação: Uma r-forma diferenciável é um tensor alternado do tipo $(\mathrm{r}, 0)$. Reciprocamente, um $(\mathrm{r}, 0)$ tensor alternado é uma $\mathrm{r}$-forma diferenciável.

Um conceito fundamental na Geometria Riemanniana é a conexão Riemanniana ou de Levi-Civita.

Proposição 2.1.6. Dada uma variedade Riemanniana M, existe uma única aplicação

$$
\nabla: \chi(\mathrm{M}) \times \chi(\mathrm{M}) \rightarrow \chi(\mathrm{M})
$$

satisfazendo às seguintes propriedades:
a)
$\nabla_{\mathrm{fX}+\mathrm{gY}} \mathrm{Z}=\mathrm{f} \nabla_{\mathrm{X}} \mathrm{Z}+\mathrm{g} \nabla_{\mathrm{Y}} \mathrm{Z}$
b) $\quad \nabla_{\mathrm{X}}(\mathrm{Y}+\mathrm{Z})=\nabla_{\mathrm{X}} \mathrm{Y}+\nabla_{\mathrm{X}} \mathrm{Z}$,
c) $\quad \nabla_{\mathrm{X}}(\mathrm{fY})=\mathrm{f} \nabla_{\mathrm{X}} \mathrm{Y}+\mathrm{X}(\mathrm{f}) \mathrm{Y}$,
d) $\mathrm{X}\langle\mathrm{Y}, \mathrm{Z}\rangle=\left\langle\nabla_{\mathrm{X}} \mathrm{Y}, \mathrm{Z}\right\rangle+\left\langle\mathrm{Y}, \nabla_{\mathrm{X}} \mathrm{Z}\right\rangle$,
e) $\quad \nabla_{\mathrm{X}} \mathrm{Y}-\nabla_{\mathrm{Y}} \mathrm{X}=[\mathrm{X}, \mathrm{Y}]$

onde $X, Y, Z \in \chi(M)$ e $f, g \in \mathbb{C}^{\infty}(M)$. Esta aplicação é denominada conexão de 
Levi-Civita (ou Riemanniana).

Observação: As três primeiras propriedades significam que $\nabla$ é um tensor na primeira variável e uma derivação na segunda variável. A quarta propriedade significa que a conexão é compatível com a métrica e a quinta propriedade que é livre de torção.

Escolhendo uma carta local $\varphi: U \rightarrow R^{n}$ em $p$, denotamos $X_{i} \in \chi(M)$ uma base de $T_{p} M$ associada. Em coordenadas locais, os campos $X, Y \in \chi(M)$ serão dados por:

$$
X=\sum_{i=1}^{n} x_{i} x_{i} \quad Y=\sum_{j=i}^{n} y_{j} X_{j}
$$

e a conexão de Levi-Cevita por:

$$
\nabla_{X} Y=\sum_{i, j}\left[x_{i} y_{j} \nabla_{X_{i}} X_{j}+x_{i} X_{i}\left(y_{j}\right) x_{j}\right]
$$

Definindo os símbolos de Christoffel da conexão por $\nabla_{X_{i}} X_{j}=\sum_{k} \Gamma_{i j}^{k} X_{k}$, onde $\Gamma_{i j}^{k} \in \mathbb{C}^{\infty}(U)$, segue que

$$
\nabla_{X} Y=\sum_{k=1}^{n}\left[\sum_{i, j=1}^{n}\left(x_{i} y_{j} \Gamma_{i j}^{k}+X\left(y_{k}\right)\right)\right] X_{k}
$$

As funções $\Gamma_{i j}^{k}$ são chamadas símbolos de Christoffel da conexão.

Como $\left[\mathrm{X}_{\mathrm{i}}, \mathrm{X}_{\mathrm{j}}\right]=0$, tem-se que

$$
\Gamma_{i j}^{k}=\Gamma_{j i}^{k} .
$$


Proposição 2.1.7 Os símbolos de Christoffel são dados por

$$
\Gamma_{i j}^{m}=\frac{1}{2} \sum_{k=1}^{n}\left\{\frac{\partial}{\partial x_{i}} g_{j k}+\frac{\partial}{\partial x_{j}} g_{k i}-\frac{\partial}{\partial x_{k}} g_{i j}\right\} g^{k m}
$$

onde $g_{i j}=\left\langle X_{i}, X_{j}\right\rangle$ e $\left(g^{\mathrm{ij}}\right)$ é a matriz inversa de $\left(\mathrm{g}_{\mathrm{ij}}\right)$.

Intrisecamente relacionado com a noção de conexão Riemanniana, está a noção de derivada covariante:

Proposição 2.1.8. Seja M uma variedade diferenciável com a conexão Riemanniana $\nabla$. Então existe uma única lei que associa a um campo vetorial diferenciável $\mathrm{V}$ ao longo da curva diferenciável

$$
\gamma: \mathrm{I} \rightarrow \mathrm{M}
$$

um outro campo vetorial diferenciável $\frac{\mathrm{DV}}{\mathrm{dt}}$ ao longo de $\gamma$, denominado derivada covariante de $\mathrm{V}$ ao longo de $\gamma$, tal que:
a) $\quad \frac{D}{d t}(V+W)=\frac{D V}{d t}+\frac{D W}{d t}$,
b) $\quad \frac{D}{d t}(f V)=\frac{d f}{d t} V+f \frac{D V}{d t}$,

onde W é um campo de vetores ao longo de $\gamma$ e $f \in \mathbb{C}^{\infty}(M)$.

c) $\quad \mathrm{Se} \mathrm{V}$ é induzido por um campo de vetores $\mathrm{Y} \in \chi(\mathrm{M})$, i.é.,

então

$$
\mathrm{V}(\mathrm{t})=\mathrm{Y}(\gamma(\mathrm{t}))
$$

$$
\frac{D V}{d t}=\nabla_{\dot{\gamma}} Y
$$


Observe que localmente todo campo de vetores ao longo de $\gamma$ é induzido por um campo em $\chi(\mathrm{M})$

Lembramos que a noção de segmento de reta unindo dois pontos, como a curva que realiza a menor distância entre estes pontos é generalizada pela noção de geodésica:

Definição 2.1.8. Uma curva parametrizada $\gamma: I \rightarrow M$ é uma geodésica se

$$
\left(\frac{D}{d t}\right) \gamma^{\prime}(t)=0^{1}
$$

para todo $\mathrm{t} \in \mathrm{I}$.

Observação: Em termos da conexão de Levi-Civita, temos localmente, $\nabla_{\dot{\gamma}} \dot{\gamma}=0$

Outro conceito fundamental na Geometria e na Mecânica Geométrica é a curvatura, tanto no caso particular de superfícies em que é descrita pela curvatura gaussiana, quanto no caso geral, descrita pelo tensor de curvatura.

Proposição 2.1.9. Sejam $\left\{X_{1}, \ldots, X_{n}\right\}$ um referencial ortonormal e $\left\{\theta_{1}, \ldots, \theta_{\mathrm{n}}\right\}$ os correferenciais ortonormais correspondentes. Então existe uma única matriz de 1-formas $\left(\omega_{\mathrm{ij}}\right)$ satisfazendo

a) $\quad \mathrm{d} \theta^{\mathrm{i}}=-\omega_{\mathrm{ij}} \wedge \theta^{\mathrm{j}}$

$1 \frac{D}{d t}$ é a derivada covariante ao longo de $\gamma$ 
b) $\quad \omega_{\mathrm{ij}}=-\omega_{\mathrm{ji}}$

Em particular, se $\mathrm{n}=2$ temos:

$$
\left(\omega_{i j}\right)=\left(\begin{array}{cc}
0 & \omega \\
-\omega & 0
\end{array}\right)
$$

Definição 2.1.10. A função $\mathrm{k}$ definida sobre uma superfície por $d \omega=-k \theta^{1} \wedge \theta^{2}$ é chamada de Curvatura Gaussiana.

A função k exprime comportamentos geométricos da superfície.

Definição 2.1.11. O tensor de Curvatura $R$ de uma variedade Riemanniana $\mathrm{M}$ é uma lei que associa a cada par $\mathrm{X}, \mathrm{Y} \in \chi(\mathrm{M})$ uma aplicação

$$
\mathrm{R}(\mathrm{X}, \mathrm{Y}): \chi(\mathrm{M}) \rightarrow \chi(\mathrm{M})
$$

dada por

$$
\mathrm{R}(\mathrm{X}, \mathrm{Y}) \mathrm{Z}=\nabla_{\mathrm{X}} \nabla_{\mathrm{Y}} \mathrm{Z}-\nabla_{\mathrm{Y}} \nabla_{\mathrm{X}} \mathrm{Z}-\nabla_{[\mathrm{X}, \mathrm{Y}]} \mathrm{Z},
$$

Proposição 2.1.12. A aplicação R é um $(3,1)$ tensor.

A demonstração se encontra em [ 1 ].

A expressão local de $\mathrm{R}$ é dada por:

$$
R\left(X_{k}, X_{1}\right) X_{j}=\nabla_{X_{k}} \nabla_{X_{1}} X_{j}-\nabla_{X_{1}} \nabla_{X_{k}} X_{j}
$$

ou, usando os símbolos de Christoffel,

$$
R\left(X_{k}, X_{1}\right) X_{j}=\sum_{i=1}^{n} R_{j k 1}^{i} X_{i}
$$

onde 


$$
R_{j k 1}^{i}=X_{1}\left(\Gamma_{k j}^{i}\right)+\sum_{m=1}^{n} \Gamma_{k j}^{m} \Gamma_{l m}^{i}-X_{k}\left(\Gamma_{1 j}^{i}\right)-\sum_{m=1}^{n} \Gamma_{l j}^{m} \Gamma_{k m}^{i}
$$

Na proposição seguinte enunciamos algumas das propriedades básicas do tensor de curvatura.

Proposição 2.1.13. O tensor curvatura $R$ goza das seguintes propriedades:
a)(Identidade de Bianchi) $\quad R(X, Y) Z+R(Y, Z) X+R(Z, X) Y=0$
notação: $R_{i 1 j k}=\left\langle R\left(X_{j}, X_{k}\right) X_{1}, X_{i}\right\rangle$,
b) $R_{i 1 j k}+R_{k 1 j i}+R_{1 j k i}=0$
c) $R_{j k 1 i}=-R_{k j 1 i}$
d) $R_{j k l i}=-R_{j k i 1}$
e) $R_{j k 1 i}=R_{1 i j k}$

Uma demonstração se encontra em [ 1 ].

Definição 2.1.14. A aplicação Ricci definida por

$$
\operatorname{Ric}(X, Y)=\text { traço da aplicação } \mathrm{V} \rightarrow \mathrm{R}(\mathrm{X}, \mathrm{V}) \mathrm{Y}
$$

onde $\mathrm{X}, \mathrm{Y}, \mathrm{V} \in \mathrm{T}_{\mathrm{p}}(\mathrm{M})$, é chamada de Curvatura Ricci. Em coordenadas locais

$$
R_{j k}=\sum_{i=1}^{n} R_{j k i}^{i}
$$


Proposição 2.1.15. Ric é um $(2,0)$ tensor simétrico.

Definição 2.1.16. A curvatura escalar é uma aplicação definida pela contração de $R_{\mathrm{jk}}$, i.e.,

$$
R=\sum_{j, k=1}^{n} g^{j k} R_{j k}
$$

Definição 2.1.17. Sejam $p \in M$ e $\sigma \subset T_{p}(M)$ um subespaço bidimensional do espaço tangente $\mathrm{T}_{\mathrm{p}}(\mathrm{M})$ e sejam $\mathrm{x}, \mathrm{y} \in \sigma$ dois vetores L.I.. $\mathrm{O}$ número real

$$
K(\sigma)=\frac{g(R(x, y) x, y)}{|x \wedge y|^{2}}
$$

onde $|x \wedge y|=\sqrt{g(x, x) g(y, y)-g(x, y)^{2}}$ é chamado Curvatura Seccional de $\sigma \mathrm{em}$

p.

Proposição 2.1.18. A Curvatura Seccional de $\sigma$ em $\mathrm{p}$ não depende da escolha da base $\{\mathrm{x}, \mathrm{y}\}$ de $\sigma$.

Uma demonstração se encontra em [ 1 ].

Pela proposição anterior, $\mathrm{k}$ está bem definida. 
Definição 2.1.19. Seja f:M $\rightarrow R$ uma aplicação diferenciável. A aplicação definida por

$$
\text { Hess } f(X, Y)=\left\langle\nabla_{X} \operatorname{grad} f, Y\right\rangle,
$$

$X, Y \in \chi(M)$, é chamada Hessiana de f.

Definição 2.1.20. O operador $\Delta$, definido por $\Delta: \mathbb{C}^{\infty}(\mathrm{M}) \rightarrow \mathbb{C}^{\infty}(\mathrm{M})$ e

$$
\Delta f=\sum_{i, j=1}^{n} g^{i j} \operatorname{Hess} f\left(X_{i}, X_{j}\right)=\operatorname{tr}(\operatorname{Hess} f),
$$

$f \in \mathbb{C}^{\infty}(\mathrm{M})$, é chamado de Laplaciano de $\mathrm{f}$. 


\section{\# 2.2. Sistema Mecânico Simples}

Nesta seção serão definidos alguns conceitos da Mecânica. A Geometria Riemanniana é usada como base para essas definições. A partir desta seção começa o elo entre a Mecânica e a Geometria de uma forma acentuada, como mostra a definição a seguir:

Definição 2.2.1. Seja $M$ uma variedade Riemanniana e V:M $\rightarrow R$ uma função diferenciável. A terna $(M, g, V)$ é chamado de Sistema Mecânico Simples. M é chamado o Espaço de Configuração, o Fibrado Tangente é o Espaço de Fase e a função V é chamada Energia Potencial do Sistema Mecânico.

A seguir, serão definidos dois conceitos básicos da Mecânica e eles serão fundamentais para o estudo dos sistemas mecânicos.

Definição 2.2.2. A função $\mathrm{K}: \mathrm{TM} \rightarrow \mathrm{R}$ definida por

$$
K(p, v)=\frac{1}{2} g_{p}(v, v), \quad(p, v) \in T M
$$

é chamada de Energia Cinética do Sistema Mecânico Simples.

Definição 2.2.3. Seja $\pi: T M \rightarrow M$ a aplicação projeção. A função E:TM $\rightarrow \mathrm{R}$ definida por 


$$
\mathrm{E}(\mathrm{p}, \mathrm{v})=\mathrm{K}(\mathrm{p}, \mathrm{v})+\mathrm{V}(\pi(\mathrm{p}, \mathrm{v}))
$$

é chamada de Energia Total.

A seguinte definição dá o conceito geométrico de movimento em um Sistema Mecânico Simples.

Definição 2.2.4. Uma curva física de um Sistema Mecânico Simples $(\mathrm{M}, \mathrm{g}, \mathrm{V})$ é uma curva diferenciável $\gamma$ em $\mathrm{M}$ tal que

$$
\left(\frac{D}{d t}\right) \gamma^{\prime}=-g r a d V
$$

Assim, as curvas físicas são as geodésicas sempre que gradV $\equiv 0$. As curvas físicas são alvos de estudo nos exemplos que estão no capítulo 4.

A seguinte proposição é relevante para o contexto, por garantir a conservação da energia ao longo de uma curva física.

Proposição 2.2.5. A Energia total E:TM $\rightarrow$ R é constante ao longo de uma curva física $\gamma(\mathrm{t})$.

Demonstração: Se $v(t)=\left(\gamma(t), \gamma^{\prime}(t)\right) \in$ TM então

$$
\mathrm{E}(\mathrm{v}(\mathrm{t}))=\mathrm{K}\left(\gamma^{\prime}(\mathrm{t})\right)+\mathrm{V}(\gamma(\mathrm{t}))=\mathrm{f}(\mathrm{t})
$$

onde $f: I \subset R \rightarrow R$.

Assim, basta mostrar que $\frac{d}{d t}(E(v(t)))=0$. Usando que a derivada 
covariante é dada pela conexão (proposição 2.1.6), obtemos

$$
\begin{aligned}
\frac{d}{d t}(E(v(t)) & =\frac{1}{2} \frac{d}{d t}\left\langle\gamma^{\prime}(t), \gamma^{\prime}(t)\right\rangle+d V\left(\gamma^{\prime}(t)\right) \\
& =\frac{1}{2}\left(\left\langle\frac{D}{d t} \gamma^{\prime}(t), \gamma^{\prime}(t)\right\rangle+\left\langle\gamma^{\prime}(t), \frac{D}{d t} \gamma^{\prime}(t)\right\rangle\right)+d V\left(\gamma^{\prime}(t)\right) \\
& =\left\langle-g r a d V, \gamma^{\prime}(t)\right\rangle+d V\left(\gamma^{\prime}(t)\right)=0 .
\end{aligned}
$$

A seguir, damos uma definição essencial para o contexto. Ela, junto com a próxima proposição, definem uma Variedade Riemanniana. Em seguida será mostrado que nesta variedade se encontram as curvas físicas de valor de energia h.

Definição 2.2.6. Seja $h \in R$. $O$ sub-conjunto $M_{h} \subset M$ definido por $\mathrm{M}_{\mathrm{h}}=\{\mathrm{p} \in \mathrm{M} ; \mathrm{V}(\mathrm{p})<\mathrm{h}\}$, é chamado $\mathrm{o}$ espaço de $\mathrm{h}$-configuração.

Proposição 2.2.7. Seja $h \in R$ um valor regular da energia potencial V.

A aplicação $g_{h}$ definida por

$$
g_{h}=2(h-V) g
$$

é uma métrica Riemanniana sobre o espaço de configuração $M_{h}$.

A demonstração segue dos fatos de que g é uma métrica Riemanniana em $\mathrm{M}$ e $\mathrm{h}-\mathrm{V}$ é diferenciável e estritamente positiva em $\mathrm{M}_{\mathrm{h}}$, fazendo-nos concluir que $\mathrm{g}_{\mathrm{h}}$ é um tensor simétrico positivo definido do tipo $(2,0)$ em $\mathrm{M}_{\mathrm{h}}$. Note que $\mathrm{g}_{\mathrm{h}} \equiv 0$ 
em $\partial M_{h}$.

Assim podemos dar a seguinte definição:

Definição 2.2.8. Seja $\rho: M \quad \rightarrow \quad R \quad$ a função definida por: $e^{2 \rho}=2(h-V)$. A terna $\left(M_{h}, g_{h}, V\right)$ é chamado de Sistema h-Mecânico Simples e $\mathrm{g}_{\mathrm{h}}=\mathrm{e}^{2 \rho} \mathrm{g}$ é chamada métrica de Jacobi.

As curvas físicas estão para a Mecânica da mesma forma que as geodésicas estão para a Geometria Diferencial. A próxima proposição dá o significado geométrico de uma curva física e para isto é feito o elo entre curvas físicas e geodésicas.

Proposição 2.2.9. (Jacobi) As curvas físicas de energia total h são, a menos de reparametrização, as geodésicas da variedade Riemanniana $\left(M_{h}, g_{h}\right)$.

Para a demonstração da proposição 2.2.8, usaremos o seguinte Lema, que dá uma importante relação entre as conexões $\nabla$ e $\nabla^{*}$ de $(M, g)$ e $\left(M_{h}, g_{h}\right)$.

Lema 2.2.10. Para todo campo de vetores $\mathrm{X}, \mathrm{Y} \in \chi(\mathrm{M})$ temos

$$
\nabla_{X}^{*} Y=\nabla_{X} Y+A_{X} Y
$$

onde

$$
A_{X} Y=d \rho(X) Y+d \rho(Y) X-g(X, Y) \operatorname{grad} \rho .
$$


Demonstração (do Lema 2.2.10):

$$
\begin{aligned}
2 e^{2 \rho} g\left(\nabla_{Y}^{*} X, Z\right)= & X\left(e^{2 \rho} g(Y, Z)\right)+Y\left(e^{2 \rho} g(Z, X)\right)-Z\left(e^{2 \rho} g(X, Y)\right)+ \\
& -e^{2 \rho} g([X, Z], Y)-e^{2 \rho} g([X, Y], Z)-e^{2 \rho} g([Y, Z], X) ;
\end{aligned}
$$

assim,

$$
\begin{aligned}
g\left(\nabla_{Y}^{*} X, Z\right) & =g\left(\nabla_{Y} X, Z\right)+X(\rho) g(Y, Z)+Y(\rho) g(Z, X)-Z(\rho) g(X, Y) \\
& =g\left(\nabla_{Y} X, Z\right)+d \rho(X) g(Y, Z)+d \rho(Y) g(Z, X)-d \rho(Z) g(X, Y) \\
& =d \rho(X) g(Y, Z)+d \rho(Y) g(Z, X)-g(g r a d \rho, Z) g(X, Y) .
\end{aligned}
$$

Portanto,

$$
\nabla_{Y}^{*} X-\nabla_{Y} X=d \rho(X) Y+d \rho(Y) X-g(X, Y) \operatorname{grad} \rho,
$$

concluindo assim a demostração do lema.

Agora estamos em condições de demonstrar a proposição 2.2 .9 como é feito a seguir.

Demonstração (da proposição 2.2.9). Seja $\gamma$ uma curva física de energia total h. Usando o lema 2.2.10

$$
\begin{aligned}
\nabla_{\gamma^{\prime}(t)}^{*} \gamma^{\prime}(t) & =\nabla_{\gamma^{\prime}(t)} \gamma^{\prime}(t)+2 \mathrm{~d} \rho\left(\gamma^{\prime}\right) \gamma^{\prime}-g\left(\gamma^{\prime}, \gamma^{\prime}\right) \text { grad } \rho \\
& =\nabla_{\gamma^{\prime}(t)} \gamma^{\prime}(t)+2 \mathrm{~d} \rho\left(\gamma^{\prime}\right) \gamma^{\prime}+\operatorname{gradV} \\
& =-\operatorname{gradV}+2 \mathrm{~d} \rho\left(\gamma^{\prime}\right) \gamma^{\prime}+\operatorname{gradV} \\
& =2 \mathrm{~d} \rho\left(\gamma^{\prime}\right) \gamma^{\prime} ;
\end{aligned}
$$

portanto,

$$
\nabla_{\gamma^{\prime}(t)} \gamma^{\prime}(t)=2 \mathrm{~d} \rho\left(\gamma^{\prime}\right) \gamma^{\prime}
$$

usando o fato de que 


$$
\begin{gathered}
d s^{\prime}=2(h-V) d t \\
\frac{d \gamma}{d s^{\prime}}=\frac{d \gamma}{d t} \frac{d t}{d s^{\prime}}=\frac{d \gamma}{d t} e^{-2 p}
\end{gathered}
$$

segue que

$$
\begin{aligned}
\nabla_{\frac{d \gamma}{d s^{\prime}} \frac{d \gamma}{d s^{\prime}}}^{*} & =\nabla_{e^{-2 \rho} \frac{d \gamma}{d t}} e^{-2 \rho} \frac{d \gamma}{d t} \\
& =e^{-2 \rho}\left[e^{-2 \rho} \nabla_{\frac{d \gamma}{d t}} \frac{d \gamma}{d t}+d\left(e^{-2 \rho}\right)\left(\frac{d \gamma}{d t}\right) \frac{d \gamma}{d t}\right] \\
& =e^{-2 \rho}\left[2 e^{-2 \rho} d \rho\left(\gamma^{\prime}\right) \gamma^{\prime}-2 e^{-2 \rho} d \rho\left(\gamma^{\prime}\right) \gamma^{\prime}\right] \\
& =0
\end{aligned}
$$

c.q.d. 


\section{\# 2.3. Conexões Projetivamente equivalentes}

Esta seção estuda casos particulares de sistemas mecânicos simples que estão relacionados através das conexões das suas respectivas variedades Riemannianas, de uma forma que é possível obter informações das geodésicas de uma variedade quando já se conhece informações das geodésicas da outra variedade Riemanniana.

Seja $\gamma: I \rightarrow M$ uma curva diferenciável em $M$ e x uma carta local em $\gamma\left(\mathrm{t}_{\mathrm{o}}\right) \operatorname{com}(\mathrm{xo \gamma})(\mathrm{t})=\left(\mathrm{x}_{1}(\mathrm{t}), \ldots, \mathrm{x}_{\mathrm{n}}(\mathrm{t})\right)$. Usando a definição de geodésica, segue que $\gamma$ é uma geodésica se e somente se, $\gamma$ satisfaz a equação

$$
\frac{d^{2} x_{i}}{d t^{2}}+\sum_{j, k=1}^{n} \Gamma_{j k}^{i} \frac{d x_{j}}{d t} \frac{d x_{k}}{d t}=0, \quad i=1, \ldots, n
$$

Efetuando uma reparametrização arbitrária $\mathrm{t}=\mathrm{t}(\tau)$, a equação 2.3. a fica da forma

2.3.b.

$$
\frac{d^{2} x_{i}}{d \tau^{2}}+\sum_{j, k=1}^{n} \Gamma_{j k}^{i} \frac{d x_{j}}{d \tau} \frac{d x_{k}}{d \tau}=-\frac{d x_{i}}{d \tau} \frac{d^{2} \tau}{d t^{2}} /\left(\frac{d \tau}{d t}\right)^{2}
$$

Proposição 2.3.1. Uma reparametrização de uma geodésica continuará sendo uma geodésica se e só se a reparametrização for linear.

Observação: Uma reparametrização de uma geodésica será denominada curva geodésica. 
Proposição 2.3.2. Duas conexões Riemannianas $\nabla$ e $\nabla^{*}$ sobre uma variedade diferenciável $\mathrm{M}$ com métricas $\mathrm{g}$ e $\mathrm{g}^{*}$ respectivamente, têm as mesmas curvas geodésicas se e somente se, existe uma 1-forma $\theta$ tal que para todo campo de vetores $\mathrm{X}, \mathrm{Y} \in \chi(\mathrm{M})$

$$
\nabla_{X}^{*} Y-\nabla_{X} Y=\theta(X) Y+\theta(Y) X,
$$

ou em coordenadas locais

$$
\Gamma_{j \mathrm{k}}^{* i}=\Gamma_{j \mathrm{k}}^{i}+\delta_{j}^{i} \theta_{\mathrm{k}}+\delta_{\mathrm{k}}^{i} \theta_{j} .
$$

Demonstração. Seja $\gamma: \mathrm{I} \rightarrow \mathrm{M}$ uma curva diferenciável em M e $\phi$ uma carta local em $\gamma\left(\mathrm{t}_{0}\right)$, tal que $\phi_{\mathrm{o}} \gamma(\mathrm{t})=\left(\mathrm{x}_{1}(\mathrm{t}), \ldots, \mathrm{x}_{\mathrm{n}}(\mathrm{t})\right)$. Assumindo que vale a equação 2.3.c, segue que

$$
\begin{aligned}
\sum_{j, k=1}^{n} \Gamma_{j k}^{* i} \dot{x}_{j} \dot{x}_{k} & =\sum_{j, k=1}^{n}\left(\Gamma_{j k}^{i}+\delta_{j}^{i} \theta_{k}+\delta_{k}^{i} \theta_{j}\right) \dot{x}_{j} \dot{x}_{k} \\
& =\sum_{j, k=1}^{n} \Gamma_{j k}^{i} \dot{x}_{j} \dot{x}_{k}+2 \sum_{k=1}^{n} \theta_{k} \dot{x}_{i} \dot{x}_{k} .
\end{aligned}
$$

Por esta razão, se $\gamma$ for uma geodésica de $\left(\mathrm{M}, \mathrm{g}^{*}\right)$, podemos usar $\mu=-2 \sum_{\mathrm{k}=1}^{\mathrm{m}} \theta_{\mathrm{k}} \dot{\mathrm{x}}_{\mathrm{k}}$ para introduzir um novo parâmetro $\tau$ tal que não só $\ddot{x}_{i}+\sum_{k, j=1}^{n} \Gamma_{j k}^{* i} \dot{x}_{j} \dot{x}_{k}=0$ mas também

$$
\frac{d^{2} x_{i}}{d \tau^{2}}+\sum_{j, k=1}^{n} \Gamma_{j k}^{i} \frac{d x_{j}}{d \tau} \frac{d x_{k}}{d \tau}-\frac{d x_{i}}{d \tau} \mu(\tau)=-\mu(\tau) \frac{d x_{i}}{d \tau}
$$


Por outro lado, se $\gamma$ for uma curva geodésica para as duas conexões dadas, então:

$$
\ddot{x}_{i}+\sum_{j, k=1}^{n} \Gamma_{j k}^{* i} \dot{x}_{j} \dot{x}_{k}=0
$$

$\mathrm{e}$

$$
\ddot{x}_{i}+\sum_{j, k=1}^{n} \Gamma_{j k}^{i} \dot{x}_{j} \dot{x}_{k}=-\lambda \dot{x}_{i}
$$

por subtração das duas últimas equações:

$$
\sum_{j, k=1}^{n}\left(\Gamma_{j k}^{* i}-\Gamma_{j k}^{i}\right) \dot{x}_{j} \dot{x}_{k}=\lambda \dot{x}_{i}
$$

e ainda

$$
\begin{aligned}
& \dot{x}_{r} \sum_{j, k=1}^{n} A_{j k}^{i} \dot{x}_{j} \dot{x}_{k}=\lambda \dot{x}_{i} \dot{x}_{r} \\
& \dot{x}_{i} \sum_{j, k=1}^{n} A_{j k}^{r} \dot{x}_{j} \dot{x}_{k}=\lambda \dot{x}_{r} \dot{x}_{i}
\end{aligned}
$$

e por uma nova subtração

$$
\dot{x}_{r_{j}} \sum_{j=1}^{n} A_{j k}^{i} \dot{x}_{j} \dot{x}_{k}-\dot{x}_{i} \sum_{j, k=1}^{n} A_{j k}^{r} \dot{x}_{j} \dot{x}_{k}=0
$$

ou

$$
\sum_{j, k=1}^{n}\left(\dot{x}_{r} A_{j k}^{i}-\dot{x}_{i} A_{j k}^{r}\right) \dot{x}_{j} \dot{x}_{k}=0
$$

portanto,

$$
0=\delta_{s}^{r} A_{j k}^{i}-\delta_{s}^{i} A_{j k}^{r}+\delta_{j}^{r} A_{k s}^{i}-\delta_{j}^{i} A_{k s}^{r}+\delta_{k}^{r} A_{s j}^{i}-\delta_{k}^{i} A_{s j}^{r}
$$


contraindo sobre $(r, s)$ segue que

$$
0=(n+1) A_{j k}^{i}-\sum_{r=1}^{n}\left(\delta_{j}^{i} A_{k r}^{r}+\delta_{k}^{i} A_{r j}^{r}\right)
$$

escrevendo $\phi_{k}=\frac{1}{n+1} \sum_{r=1}^{n} A_{k r}^{r}$

segue que $A_{j k}^{i}=\delta_{j}^{i} \phi_{k}+\delta_{k}^{i} \phi_{j}$

$$
\text { c.q.d. }
$$

Agora estamos em condições de dar a seguinte definição:

Definição 2.3.3. Duas conexões Riemannianas da variedade $M$, com métricas g e $\mathrm{g}^{*}$ que têm as mesmas curvas geodésicas são chamadas projetivamente equivalentes.

Quando tivermos duas conexões projetivamente equivalentes, a proposição 2.3.2 fornece uma relação entre elas. Para explicitarmos a forma lá definida, faremos a contração na equação 2.3.c com relação a (i,j):

$$
\sum_{i=1}^{n}\left(\Gamma_{i k}^{* i}-\Gamma_{i k}^{i}\right)=\sum_{i=1}^{n}\left(\theta_{k}+\delta_{k}^{i} \theta_{i}\right)
$$

ou

$$
\frac{1}{2} X_{k} \ln \frac{\left|g^{\prime}\right|}{|g|}=\sum_{i=1}^{n}\left(\theta_{k}+\delta_{k}^{i} \theta_{i}\right)^{2}
$$

e portanto

${ }^{2}|g|$ é o determinante da matrix $\left(g_{i j}\right)$ 


$$
\theta_{k}=\frac{1}{2(n+1)} X_{k} \ln \frac{\left|g^{\prime}\right|}{|g|}
$$

De uma forma mais geral, dizemos que duas variedades Riemannianas $(M, g)$ e $\left(M, g^{\prime}\right)$ são projetivamente equivalentes se, e somente se, suas respectivas conexões são projetivamente equivalentes.

Definição 2.3.4. Dois Sistemas Mecânicos Simples (M,g, V) e (M, $\left.{ }^{*}, V^{*}\right)$ são chamados equivalentes para $\left(\mathrm{h}, \mathrm{h}^{*}\right)$ se existe um difeomorfismo $\phi$ levando curvas geodésicas de $\left(\mathrm{M}_{\mathrm{h}}, \mathrm{g}_{\mathrm{h}}\right)$ sobre curvas geodésicas de $\left(\mathrm{M}_{\mathrm{h}^{*}}, \mathrm{~g}_{\mathrm{h}^{*}}^{*}\right)$.

Denotaremos as conexões de $\left(\mathrm{M}_{\mathrm{h}}, \mathrm{e}^{2 \rho} \mathrm{g}\right)$ e $\left(\mathrm{M}_{\mathrm{h}^{\prime}}, \mathrm{e}^{2 \rho^{\prime}} \mathrm{g}^{\prime}\right)$ por $\nabla^{*}$ e $\nabla^{\prime *}$ respectivamente onde $\mathrm{e}^{2 \rho}=2(\mathrm{~h}-\mathrm{V})$ e $\mathrm{e}^{2 \rho^{\prime}}=2\left(\mathrm{~h}^{\prime}-\mathrm{V}^{\prime}\right) . \operatorname{Se}\left(\mathrm{M}_{\mathrm{h}}, \mathrm{e}^{2 \rho} \mathrm{g}\right)$ e $\left(\mathrm{M}_{\mathrm{h}^{\prime}}, \mathrm{e}^{2 \rho^{\prime}} \mathrm{g}^{\prime}\right)$ são projetivamente equivalentes, então existe uma 1 -forma $\theta$ tal que

$$
\nabla_{X}^{*} Y-\nabla_{X}^{* \prime} Y=\theta(X) Y+\theta(Y) X
$$

onde $\theta=d \varphi$ e

$$
\varphi=\frac{1}{2(\mathrm{n}+1)} \ln \frac{\left|\mathrm{e}^{2 \rho^{\prime}} \mathrm{g}^{\prime}\right|}{\left|\mathrm{e}^{2 \rho} \mathrm{g}\right|}
$$

assim

$$
\begin{aligned}
d \varphi & =\frac{1}{2(n+1)}\left(2 n d \rho^{\prime}+d \ln \left|g^{\prime}\right|-2 n d \rho-d \ln |g|\right) \\
& =\frac{n}{(n+1)}\left(d\left(\rho^{\prime}-\rho\right)\right)+d \psi
\end{aligned}
$$


com

$$
\begin{aligned}
& \varphi=\frac{n}{n+1}\left(\rho^{\prime}-\rho\right)+\psi \\
& \psi=\frac{1}{2(n+1)} \ln \frac{\left|g^{\prime}\right|}{|g|}
\end{aligned}
$$

Usando o lema 2.2.10 segue que:

2.3.d. $\quad \nabla_{X}^{*} Y=\nabla_{X} Y+d \rho(X) Y+d \rho(Y) X-g(X, Y)$ gradp

2.3.e. $\quad \nabla_{X}^{* \prime} Y=\nabla_{X}^{\prime} Y+d \rho^{\prime}(X) Y+d \rho^{\prime}(Y) X-g^{\prime}(X, Y) \operatorname{grad}^{\prime} \rho^{\prime}{ }^{3}$

subtraindo (2.3.d) de (2.3.e) segue que:

$$
\begin{aligned}
\nabla_{X}^{*} Y-\nabla_{X}^{*^{\prime}} Y= & \nabla_{X} Y-\nabla_{X}^{\prime} Y+d\left(\rho-\rho^{\prime}\right)(X) Y+d\left(\rho-\rho^{\prime}\right)(Y) X+ \\
& -g(X, Y) \operatorname{grad} \rho+g^{\prime}(X, Y) \operatorname{grad}^{\prime} \rho^{\prime}
\end{aligned}
$$

Assumindo que $\nabla_{X}^{*} Y$ e $\nabla_{X}^{* \prime} Y$ são projetivamente equivalentes, de acordo com o que foi visto acima, segue que

$$
\begin{aligned}
& \frac{1}{\mathrm{n}+1}\left[d\left(\rho-\rho^{\prime}\right)(X) Y+d\left(\rho-\rho^{\prime}\right)(Y) X\right]-g(X, Y) \operatorname{grad} \rho \\
+ & g^{\prime}(X, Y) \operatorname{grad}^{\prime} \rho^{\prime}+\nabla_{X} Y-\nabla_{X}^{\prime} Y-d \psi(X) Y-d \psi(Y) X=0
\end{aligned}
$$

Toda ferramenta elaborada acima é para que a questão de equivalência dos sistemas mecânicos simples $(M, g, V)$ e $\left(M, g^{\prime}, V^{\prime}\right)$ fosse reduzida ao estudo da aplicação $\Theta: \chi(\mathrm{M}) \times \chi(\mathrm{M}) \rightarrow \chi(\mathrm{M})$, definida por 


$$
\begin{aligned}
& \Theta(X, Y)=\frac{1}{n+1}\left[d\left(\rho-\rho^{\prime}\right)(X) Y+d\left(\rho-\rho^{\prime}\right)(Y) X\right]+ \\
& -g(X, Y) g r a d \rho+g^{\prime}(X, Y) g r a d^{\prime} \rho^{\prime} \\
& O \text { próximo resultado significa que } \Theta \text { é tensorial, e isto é um ponto }
\end{aligned}
$$
fundamental para o contexto, pois além de resumir importantes informações, ainda torna as equações covariantes nos sistemas envolvidos.

Proposição 2.3.6. A aplicação $\Theta: \chi(\mathrm{M}) \times \chi(\mathrm{M}) \rightarrow \chi(\mathrm{M})$ definida acima é um $(2,1)$ tensor simétrico.

Demonstração: A simetria segue do fato que g e g' são simétricos.

A linearidade segue usando que a diferencial e as métricas são tensores.

$$
\text { c.q.d. }
$$

O resultado a seguir relata a importância do tensor $\Theta$ para esta questão de equivalência

Proposição 2.3.7. Suponhamos que (M,g) e (M,g') são projetivamente equivalentes. Então $\left(\mathrm{M}, \mathrm{e}^{2 \rho} \mathrm{g}\right)$ e $\left(\mathrm{M}, \mathrm{e}^{2 \rho^{\prime}} \mathrm{g}^{\prime}\right)$ são projetivamente equivalentes se, e somente se $\theta \equiv 0$.

A demonstração da proposição segue da própria definição de $\Theta$ e do que foi visto acima.

$$
\text { c.q.d. }
$$


Como a questão de equivalência depende exclusivamente das hipóteses da energia cinética, a seguir é dado uma importante relação entre o tensor $\theta$ e as métricas de Jacobi no caso em que consideramos $\mathrm{g}=\mathrm{g}$ '

Proposição 2.3.8. Seja M conexo. Dois Sistemas Mecânicos Simples $(\mathrm{M}, \mathrm{g}, \mathrm{V})$ e (M,g,V') são projetivamente equivalentes para (h,h') se, e somente se,

$$
\mathrm{h}-\mathrm{V}=\mathrm{c}\left(\mathrm{h}^{\prime}-\mathrm{V}^{\prime}\right)
$$

onde c é uma constante.

Demonstração:

$$
\Theta \equiv 0 \text { se, e somente se } \mathrm{d}\left(\rho-\rho^{\prime}\right) \equiv 0
$$

tomando:

$$
\mathrm{e}^{2 \rho}=2(\mathrm{~h}-\mathrm{V}) \text { e } \mathrm{e}^{2 \rho^{\prime}}=2\left(\mathrm{~h}^{\prime}-\mathrm{V}^{\prime}\right)
$$

segue que:

$\Theta \equiv 0$ se, e somente se $\mathrm{h}-\mathrm{V}=\mathrm{c}\left(\mathrm{h}^{\prime}-\mathrm{V}^{\prime}\right)$, onde $\mathrm{c}$ é uma constante. c.q.d. 


\section{Capítulo 3}

\section{Os Tensores Mecânicos}

\section{\# 3.0. Introdução}

Para estudar os tensores mecânicos da variedade diferenciável $\mathrm{M}_{\mathrm{h}}$, nada mais natural do que começar pelas suas relações com os tensores de M. O objetivo é facilitar a descrição da geometria de $M$ estudando a geometria de $M_{h}$.

A seção 1 é o estudo do tensor mecânico $R^{*}$ da variedade $M_{h}$. É conveniente neste estudo introduzir um operador auto-adjunto cujos auto-valores dão informações sobre $\mathrm{R}^{*}$.

A segunda seção, contém um estudo do tensor Ric ${ }^{*}$ e a curvatura escalar mecânica que são obtidos por contração do tensor mecânico de Riemann. No final da seção, ainda usando o tensor de Riemann, é introduzida a curvatura seccional mecânica. A seção 3 contém proposições dando informações sobre os sinais das curvaturas.

Dentro da seção quatro, está entre outras, a definição das funções hcurvatura mecânica. Elas são usadas como ferramentas para uma análise de sinais das curvaturas. 
Finalizando, a seção 5 trata de um estudo dos sinais da curvatura, agora em uma vizinhança dos pontos críticos do potencial V.

\section{\# 3.1. O tensor Curvatura h-Mecânico $\mathbf{R}^{*}$.}

Esta seção contém cálculos envolvendo os tensores de curvatura de Riemann $R$ e $R^{*}$ das variedades diferenciáveis $M$ e $M_{h}$, respectivamente. Nestes cálculos, são obtidos resultados que serão usados para uma primeira análise das demais curvaturas de $M_{h}$.

Para obter uma relação entre os tensores $R$ e $R^{*}$ das variedades $M$ e $M_{h}$ respectivamente, é essencial recorrer ao lema 2.2.10, como é visto a seguir.

Sejam $\nabla$ e $\nabla^{*}$ as conexões do sistema mecânico simples $(\mathrm{M}, \mathrm{g}, \mathrm{V})$ e do sistema h-Mecânico simples $\left(\mathrm{M}_{\mathrm{h}}, \mathrm{g}_{\mathrm{h}}, \mathrm{V}\right)$ respectivamente (veja definição 2.2.8).

Com estas notações, usando a definição 2.1.8, segue que:

$$
\begin{aligned}
& R_{j k 1}^{* i}-R_{j k 1}^{i}=X_{1}\left(\Gamma_{k j}^{* i}-\Gamma_{k j}^{i}\right)-X_{k}\left(\Gamma_{1 j}^{* i}-\Gamma_{1 j}^{i}\right)+ \\
& +\sum_{m=1}^{n}\left[\Gamma_{k j}^{* m} \Gamma_{l m}^{* i}-\Gamma_{1 j}^{* m} \Gamma_{k m}^{* i}+\Gamma_{1 j}^{m} \Gamma_{k m}^{i}-\Gamma_{k j}^{m} \Gamma_{1 m}^{i}\right]
\end{aligned}
$$

e desta última expressão, usando o lema 2.2.10, 


$$
\begin{aligned}
& R_{j k 1}^{* i}-R_{j k l}^{i}=\rho_{1 k} \delta_{j}^{i}+\rho_{1 j} \delta_{k}^{i}-g_{k j} X_{1}\left(\rho^{i}\right)-\rho^{i} X_{1}\left(g_{k j}\right)-\rho_{k l} \delta_{j}^{i}-\rho_{j k} \delta_{1}^{i}+ \\
& +\rho^{i} X_{k}\left(g_{1 j}\right)+g_{1 j} X_{k}\left(\rho^{i}\right)+\Gamma_{k j}^{i} \rho_{1}+\sum_{m} \Gamma_{k j}^{m} \rho_{m} \delta_{l}^{i}-\sum_{m} g_{1 m} \rho^{i} \Gamma_{k j}^{m}+ \\
& +\rho_{k} \Gamma_{l j}^{i}+\rho_{k} \rho_{1} \delta_{i}^{j}+\rho_{k} \rho_{j} \delta_{1}^{i}-\rho^{i} \rho_{k} g_{l j}+\rho_{j} \Gamma_{1 k}^{i}+\rho_{j} \rho_{1} \delta_{k}^{i}+\rho_{j} \rho_{k} \delta_{l}^{i}+ \\
& -\rho_{j} \rho^{i} g_{l k}-\sum_{m} g_{k j} \rho^{m} \Gamma_{l m}^{i}-g_{k j} \rho^{i} \rho_{1}-\sum_{m} g_{k j} \rho^{m} \rho_{m} \delta_{1}^{i}+\sum_{m} \rho^{m} g_{l m} \rho^{i} g_{k j}+ \\
& -\sum_{\mathrm{m}} \rho_{\mathrm{m}} \Gamma_{1 j}^{\mathrm{m}} \delta_{\mathrm{k}}^{i}+\sum_{\mathrm{m}} \Gamma_{1 j}^{\mathrm{m}} \mathrm{g}_{\mathrm{km}} \rho^{i}-\Gamma_{1 j}^{i} \rho_{k}-\rho_{1} \Gamma_{\mathrm{kj}}^{i}-\rho_{1} \rho_{k} \delta_{j}^{i}-\rho_{1} \rho_{j} \delta_{k}^{i}+ \\
& -\rho_{1} \rho_{j} \delta_{k}^{i}+\rho_{1} g_{k j} \rho^{i}-\rho_{j} \Gamma_{k l}^{i}-\rho_{j} \rho_{k} \delta_{1}^{i}-\rho_{j} \rho_{1} \delta_{k}^{i}+\rho_{j} g_{1 k} \rho^{i}+ \\
& +\sum_{m} g_{1 j} \rho^{m} \Gamma_{k m}^{i}+g_{1 j} \rho^{i} \rho_{k}+\sum_{m} g_{l j} \rho^{m} \rho_{m} \delta_{k}^{i}-\sum_{m} g_{1 j} \rho^{m} g_{k m} \rho^{i}
\end{aligned}
$$

ou ainda

$$
\begin{aligned}
R_{j k l}^{* i}-R_{j k l}^{i} & =\left(\rho_{j l}-\sum_{m} \rho_{m} \Gamma_{l j}^{m}-\rho_{l} \rho_{j}+\sum_{m} g_{l j} \rho^{m} \rho_{m}\right) \delta_{k}^{i}+ \\
& +\left(-\rho_{j k}+\sum_{m} \Gamma_{k j}^{m} \rho_{m}+\rho_{j} \rho_{k}-\sum_{m} g_{k j} \rho^{m} \rho_{m}\right) \delta_{l}^{i}+ \\
& +g_{k j}\left(-\sum_{m} g^{i m} \rho_{1 m}+\sum_{m, s} \rho_{m} \Gamma_{l s}^{m} g^{i s}+\sum_{m} \rho^{m} g_{l m} \rho^{i}\right)+ \\
& +g_{l j}\left(\sum_{m} g^{i m} \rho_{k m}-\sum_{m, s} \rho_{m} \Gamma_{k s}^{m} g^{i s}-\sum_{m} \rho^{m} g_{k m} \rho^{i}\right)
\end{aligned}
$$

De uma forma natural, e até por um fator simplificador, é conveniente introduzir as seguintes notações:

$$
S_{j k l}^{i}=R_{j k l}^{* i}-R_{j k l}^{i}
$$

onde 


$$
\begin{aligned}
S_{j k 1}^{i}= & -\rho_{[j k]} \delta_{1}^{i}+\rho_{[j]]} \delta_{k}^{i}-g_{j k} \rho_{[1]}^{[i]}+g_{j l} \rho_{[k]}^{[i]}+ \\
& -\langle d \rho, d \rho\rangle\left(g_{j k} \delta_{l}^{i}-g_{j l} \delta_{k}^{i}\right)
\end{aligned}
$$

sendo

$$
\rho_{[j k]}=\rho_{j k}-\sum_{m} \rho_{m} \Gamma_{j k}^{m}-\rho_{j} \rho_{k}
$$

e

$$
\rho_{[1]}^{[i]}=\sum_{m} g^{i m} \rho_{[m 1]}
$$

Observação:

1) $S_{j k 1}^{i}$ é um tensor. Isto segue do fato de que a diferença de tensores

também é um tensor.

2) As relações entre $R$ e $R^{*}$ se tornam mais simples quando as conexões são projetivamente equivalentes, já que neste caso vale

$$
\Gamma_{j k}^{* i}=\Gamma_{j k}^{i}+v_{j} \delta_{k}^{i}+v_{k} \delta_{j}^{i}
$$

No estudo da relação entre a curvatura de $\mathrm{M}$ e $\mathrm{M}_{\mathrm{h}}$, será útil decompor $S_{j k l}^{i} \quad$ como soma de tensores. Para isto é conveniente introduzir a aplicação

$$
\mathrm{C}: \chi(\mathrm{M}) \times \chi(\mathrm{M}) \rightarrow \mathrm{R}
$$

definida por 


$$
C(X, Y)=-(n-2)\left[\operatorname{Hess} \rho(X, Y)-d \rho(X) d \rho(Y)+\frac{1}{2}\langle d \rho, d \rho\rangle\langle X, Y\rangle\right]
$$

Localmente,

$$
C\left(X_{1}, X_{k}\right)=-(n-2)\left[\operatorname{Hess} \rho\left(X_{1}, X_{k}\right)-d \rho\left(X_{1}\right) d \rho\left(X_{k}\right)+\frac{1}{2}\langle d \rho, d \rho\rangle\left\langle X_{1}, X_{k}\right\rangle\right]
$$

ou ainda

$$
C_{1 k}=-(n-2)\left[\rho_{[1 k]}+\frac{1}{2}\left\langle d \rho, d \rho>g_{1 k}\right]\right.
$$

A seguinte proposição é um resultado importante no estudo do tensor hmecânico $\mathrm{R}^{*}$ e assim dos demais tensores curvatura.

Proposição 3.1.1. C é um $(2,0)$ tensor simétrico.

Demonstração. Sejam $X, Y, Z \in \chi(M)$ e f,g $\in \mathbb{C}^{\infty}(M)$, assim

$$
\begin{aligned}
\text { Hess } \rho(f X+g Z, Y) & =\left\langle\nabla_{f X+g Z} g r a d \rho, Y\right\rangle=\left\langle f \nabla_{X} \operatorname{grad} \rho, Y\right\rangle+\left\langle g \nabla_{Z} \operatorname{grad} \rho, Y\right\rangle \\
& =f H e s s(X, Y)+\operatorname{gHess} \rho(Z, Y)
\end{aligned}
$$

e com o mesmo raciocínio,

$$
\operatorname{Hess} \rho(X, f Z+g Y)=f H \operatorname{Hess} \rho(X, Z)+g \operatorname{Hess} \rho(X, Y) \text {. }
$$

Logo, a Hessiana de $\rho$ é bilinear, diferenciável por definição e, portanto, um tensor do tipo $(2,0)$. Portanto, a demonstração de que $C$ é um $(2,0)$ tensor segue do fato que as outras duas parcelas da aplicação C são tensores do tipo $(2,0)$.

Para provar a simetria, basta observar 
3.1.a.

$$
\operatorname{Hess} \rho\left(X_{j}, X_{k}\right)=\rho_{j k}-\sum_{m=1}^{n} \rho_{m} \Gamma_{j k}^{m}=\operatorname{Hess} \rho\left(X_{k}, X_{j}\right)
$$

3.1.b.

$$
d \rho\left(X_{j}\right) d \rho\left(X_{k}\right)=d \rho\left(X_{k}\right) d \rho\left(X_{j}\right)
$$

3.1.c.

$$
\left\langle x_{j}, x_{k}\right\rangle=\left\langle x_{k}, x_{j}\right\rangle
$$

c.q.d

\section{Vamos definir}

$$
\begin{aligned}
C_{k}^{j} & =\sum_{m} g^{m j} C_{m k} \\
& =\sum_{m} g^{j m}\left[-(n-2) \rho_{[m k]}+\frac{1}{2}\langle d \rho, d \rho\rangle g_{m k}\right] \\
& =-(n-2)\left[\rho[k]+\frac{1}{2}\langle d \rho, d \rho\rangle \delta_{k}^{j}\right]
\end{aligned}
$$

A partir desta última expressão, $S_{j k 1}^{i}$ pode ser escrito como uma combinação linear do $(2,0)$ tensor simétrico C, como segue:

$$
\begin{aligned}
S_{j k 1}^{i} & =-\rho_{[j k]} \delta_{1}^{i}+\rho_{[j 1]} \delta_{k}^{i}-g_{j k} \rho_{[1]}^{[i]}+g_{j 1} \rho_{[k]}^{[i]}-\langle d \rho, d \rho\rangle\left\langle g_{j k} \delta_{1}^{i}-g_{j 1} \delta_{k}^{i}\right) \\
& =\frac{1}{n-2}\left[C_{j k} \delta_{1}^{i}-C_{j 1} \delta_{k}^{i}+g_{j k} C_{1}^{i}-g_{j 1} C_{k}^{i}\right]
\end{aligned}
$$

Definição 3.1.2. Definimos o operador linear $\mathrm{C}^{*}$ em $\chi(\mathrm{M})$, por

$$
\left\langle\mathrm{C}^{*} \mathrm{X}, \mathrm{Y}\right\rangle=\mathrm{C}(\mathrm{X}, \mathrm{Y})
$$

para todo $\mathrm{X}, \mathrm{Y} \in \chi(\mathrm{M})$. Note que, da simetria de $\mathrm{C}$, segue que $\mathrm{C}^{*}$ é auto-adjunto. Localmente segue que: 


$$
\begin{gathered}
R^{*}\left(x_{j}, x_{k}\right) x_{1}-R\left(x_{j}, x_{k}\right) x_{1}=\sum_{i} S_{j k l}^{i} X_{i} \\
=\frac{1}{n-2}\left[C_{1 k} X_{j}-C_{j l} x_{k}+g_{1 k} \sum_{i, s} g^{i s} C_{s j} x_{i}-g_{j l} \sum_{i s} g^{i s} C_{s k} x_{i}\right] \\
=\frac{1}{n-2}\left[C\left(x_{1}, x_{k}\right) x_{j}-C\left(x_{j}, x_{1}\right) x_{k}+g_{1 k} C^{*} X_{j}-g_{j l} C^{*} x_{k}\right]
\end{gathered}
$$

ou seja, para a análise do tensor curvatura, basta-nos nos restringirmos à análise do operador auto-adjunto $\mathrm{C}^{*}$. 


\section{\# 3.2. O tensor Ric*, Curvatura Escalar}

\section{Mecânica e a Curvatura Seccional Mecânica.}

Nesta seção, baseando-nos em resultados da seção 3.1, introduzimos as Curvaturas de Ricci, Escalar e Seccional Mecânica. Essas curvaturas dão informações sobre a geometria da variedade $\mathrm{M}_{\mathrm{h}}$. Para obter a diferença Ric ${ }^{*}$ - Ric, basta contrair $S_{j k 1}^{i}$ (def. 2.1.14) com respeito a (i,l).

$$
\begin{aligned}
S_{j k} & =\sum_{i} S_{j k i}^{i} \\
& =-n \rho_{[j k]}+\rho_{[j k]}-g_{j k} \sum_{m, i} g^{i m} \rho_{[i m]}+\rho_{[j k]}-n\langle d \rho, d \rho\rangle g_{j k} \\
& =-(n-2) \rho_{[j k]}-[(n-2)\langle d \rho, d \rho\rangle+\Delta \rho] g_{j k}
\end{aligned}
$$

e portanto

$$
\operatorname{RiC}^{*}\left(X_{j}, X_{k}\right)-\operatorname{RiC}\left(X_{j}, X_{k}\right)=-(n-2) \rho_{[j k]}-[(n-2)\langle d \rho, d \rho\rangle+\Delta \rho] g_{j k}
$$

A curvatura escalar é obtida por mais uma contração (def.2.1.16),

$$
R^{*}=\sum_{i, j} g^{* i j} R_{i j}^{*}=e^{-2 \rho}[R+S]
$$

onde

$$
\begin{aligned}
g_{i j}^{*} & =e^{2 \rho} g_{i j} \\
g^{* i j} & =e^{-2 \rho} g^{i j}
\end{aligned}
$$

$\mathrm{e}$ 


$$
\begin{aligned}
\text { 3.2.a. } & =\sum_{m, j} g^{m j} C_{m j} \\
& =-(n-2) \Delta \rho+(n-2)\langle d \rho, d \rho\rangle-[(n-2)\langle d \rho, d \rho\rangle+\Delta \rho] n \\
& =-2(n-2) \Delta \rho-(n-2)(n-1)\langle d \rho, d \rho\rangle
\end{aligned}
$$

Assim

$$
e^{2 \rho} R^{*}-R=S
$$

Substituindo $\mathrm{e}^{2 \rho}=2(\mathrm{~h}-\mathrm{V})$, é possível escrever algumas equações mencionadas acima em função do potencial V.

Usando as relações:

$$
e^{2 \rho} d \rho=-d V
$$

e

$$
\begin{aligned}
\operatorname{Hess} \rho(X, Y) & =\left(\nabla_{X}-e^{-2 \rho} d V\right) Y \\
& =X\left(-e^{-2 \rho} d V(Y)\right)+e^{-2 \rho} d V\left(\nabla_{X} Y\right) \\
& =-d V(Y) X\left(e^{-2 \rho}\right)-e^{-2 \rho X}(d V(Y))+e^{-2 \rho} d V\left(\nabla_{X} Y\right) \\
& =-d V(Y) 2 e^{-4 \rho} d V(X)-e^{-2 \rho}\left(\nabla_{X} d V\right) Y \\
& =-2 e^{-4 \rho} d V(Y) d V(X)-e^{-2 \rho} \operatorname{HesS} V(X, Y)
\end{aligned}
$$

segue que 
3.2.b

$$
\begin{aligned}
C(X, Y)= & -(n-2)\left[-2 e^{-4 p} d V(X) d V(Y)+\right. \\
& -e^{-2 \rho} \operatorname{HessV}(X, Y)-e^{-4 \rho} d V(X) d V(Y)+ \\
& \left.+\frac{1}{2} e^{-4 \rho}\langle d V, d V\rangle\langle X, Y\rangle\right] \\
= & \frac{(n-2)}{4(h-V)^{2}}[3 d V(X) d V(Y)+2(h-V) \operatorname{HessV}(X, Y)+ \\
& -\frac{1}{2} N_{V^{2}}\langle X, Y\rangle
\end{aligned}
$$

onde $N_{\mathrm{V}^{2}}=\langle d V, d V\rangle$ é chamada a norma do potencial V.

\section{Por definição,}

$$
\begin{aligned}
\Delta \rho= & \operatorname{tr} \operatorname{hess} \rho=\operatorname{tr}\left[\left(-\mathrm{e}^{-2 \rho} \nabla_{\mathrm{X}} \mathrm{dV}\right) \mathrm{Y}+\right. \\
& \left.+\left(-2 \mathrm{e}^{-4 \rho}\langle\operatorname{dV}(\mathrm{X}) \operatorname{gradV}, \mathrm{Y}\rangle\right)\right] \\
= & -\frac{2}{\mathrm{e}^{4 \rho}} \mathrm{N}_{\mathrm{V}^{2}}-\frac{1}{\mathrm{e}^{2 \rho}} \Delta V .
\end{aligned}
$$

3.2.c.

Usando esta última relação na expressão de Ric, segue que

$$
\begin{aligned}
\operatorname{RiC}^{*}(X, Y) & -\operatorname{RiC}(X, Y)=-(n-2)\left[-\frac{2}{e^{4 p}} d V(X) d V(Y)+\right. \\
& \left.-\frac{1}{e^{2 \rho}} \operatorname{Hess} V(X, Y)-e^{-4 p} d V(X) d V(Y)\right]+ \\
& -\left[(n-2) e^{-4 p}\langle d V, d V\rangle-2 e^{-4 p} N V^{2}-e^{-2 p} \Delta V\right]\langle X, Y\rangle .
\end{aligned}
$$

ou ainda

$$
\begin{aligned}
\operatorname{RiC}^{*}(X, Y) & -\operatorname{RiC}(X, Y)=-(n-2) e^{-4 \rho}[3 d V(X) d V(Y)+ \\
& +e^{2 \rho} \operatorname{Hess} V(X, Y)+e^{-4 \rho}\left[-(n-4) N_{V^{2}}+\right. \\
& \left.+e^{2 \rho} \Delta V\right]\langle X, Y\rangle .
\end{aligned}
$$


Assim,

$$
\begin{aligned}
\operatorname{RiC}^{*}(\mathrm{X}, \mathrm{Y}) & -\operatorname{RiC}(\mathrm{X}, \mathrm{Y})=\frac{1}{4(\mathrm{~h}-\mathrm{V})^{2}}[2(\mathrm{~h}-\mathrm{V})[(\mathrm{n}-2) \text { HessV }+ \\
& +\Delta \mathrm{V}\langle\mathrm{X}, \mathrm{Y}\rangle]-(\mathrm{n}-4) \mathrm{N}_{\mathrm{v}^{2}}\langle\mathrm{X}, \mathrm{Y}\rangle+ \\
& +3(\mathrm{n}-2) \mathrm{dV}(\mathrm{X}) \mathrm{dV}(\mathrm{Y})]
\end{aligned}
$$

e portanto

$$
\begin{aligned}
S_{j k}= & \frac{1}{4(h-V)^{2}}\left[2(h-V)\left[(n-2) v_{j ; k}+\Delta V g_{j k}\right]+\right. \\
& \left.-(n-4) N_{v^{2}} g_{j k}+3(n-2) V_{j} v_{k}\right]
\end{aligned}
$$

Para obter a curvatura escalar mecânica $R^{*}$ em função de $V$, procede-se da mesma forma, substituindo 3.2.c na expressão 3.2.a, isto é,

3.2.d

$$
\begin{aligned}
e^{2 \rho} R^{*}= & R-2(n-1)\left[-\frac{2}{e^{4 \rho}} N_{v^{2}}-\frac{1}{e^{2 \rho}} \Delta V+\right. \\
& \left.+\frac{n-2}{2}\left\langle-e^{-2 \rho} d V,-e^{-2 \rho} d V\right\rangle\right] \\
= & R+\frac{2}{e^{4 \rho}}(n-1)\left[e^{2 \rho} \Delta V+\frac{n-6}{2} N^{2}\right] \\
& =R+\frac{n-1}{e^{4 \rho}}\left[2(h-V) \Delta V+\frac{n-6}{2} N^{2}\right]
\end{aligned}
$$

As curvaturas mecânicas são importantes ferramentas para a análise das curvas físicas de um sistema mecânico. Entre elas a mais importante é a curvatura seccional mecânica; ela é uma generalização da curvatura gaussiana e é obtida considerando o espaço bidimensional $\pi$, gerado pelos vetores linearmente independentes $\{\mathrm{x}, \mathrm{y}\}$ e a definição de $\mathrm{K}$. Novamente será usado o tensor C, sendo 
desta vez para escrever a curvatura Seccional como é feito a seguir:

$3.2 . e$

$$
\begin{aligned}
e^{2 \rho} \mathrm{K}^{*}(\pi) & -\mathrm{K}(\pi)=\frac{g\left(R^{*}(X, Y) Y-R(X, Y) Y, X\right)}{|X \wedge Y|^{2}} \\
= & \frac{1}{|X \wedge Y|^{2}} \frac{1}{n-2}[C(Y, Y) g(X, X)-C(X, Y) g(Y, X)+ \\
& +C(X, X) g(Y, Y)-g(X, Y) C(X, Y)] \\
= & \frac{1}{(n-2)|X \wedge Y|^{2}}[-2 C(X, Y) g(X, Y)+C(Y, Y) g(X, X)+ \\
& +C(X, X) g(Y, Y)]
\end{aligned}
$$




\section{\# 3.3. O sinal da Curvatura Mecânica}

Nesta seção serão analisados os sinais das curvaturas mecânicas em uma vizinhança do bordo de $\mathrm{M}_{\mathrm{h}}$. Será visto que o módulo das curvaturas h-mecânicas vão para o infinito quando $\mathrm{x} \rightarrow \mathrm{M}_{\mathrm{h}}$; geometricamente, isto quer dizer que as geodésicas tendem para o bordo de $\mathrm{M}_{\mathrm{h}}$.

Como os ponto do bordo de $M_{h}$ são valores regulares de $V$, existe uma vizinhança do bordo em que o gradiente de $\mathrm{V}$ é diferente de zero.

Vamos supor que $M$ seja conexa e $M_{h}$ tenha o bordo não vazio. As proposições a seguir exprimem comportamentos geométricos próximos do bordo de $M_{h}$.

Proposição 3.3.1. Quando $x \rightarrow \partial M_{h}$,

a) $\quad R^{*}(x) \rightarrow \infty$ se $\mathrm{n}<6$

b) $\quad R^{*}(x) \rightarrow-\infty$ se $\mathrm{n}>6$

c) $\left|R^{*}(x)\right| \rightarrow \infty$ se $\mathrm{n}=6$ e $\Delta \mathrm{V}$ não é nulo em uma vizinhança de $\partial \mathrm{M}_{\mathrm{h}}$.

Demonstração. Tomando $\mathrm{L}=2(\mathrm{~h}-\mathrm{V}) \Delta \mathrm{V}-(\mathrm{n}-6) \mathrm{N}_{\mathrm{v} 2}$, sobre o bordo de $\mathrm{M}_{\mathrm{h}}$,

$$
L=-(n-6) N_{v 2} \text {. }
$$

Assim 


$$
\begin{aligned}
& \mathrm{L}>0 \text { se } \mathrm{n}<6 \\
& \mathrm{~L}<0 \text { se } \mathrm{n}>6 \\
& \mathrm{~L}=0 \text { se } \mathrm{n}=6
\end{aligned}
$$

Usando que $\mathrm{e}^{6 \rho} \rightarrow 0$ quando $\mathrm{x} \rightarrow \partial \mathrm{M}_{\mathrm{h}}$, e também a continuidade de $\Delta \mathrm{V}$, da equação 3.2.d seguem (a), (b) e (c).

$$
\text { c.q.d. }
$$

Proposição 3.3.2. Se $\mathrm{V}$ é harmônica (isto é $\Delta \mathrm{V}=0$ ), então em uma vizinhança de $\partial \mathrm{M}_{\mathrm{h}}$
a) $\quad \mathrm{R}>0 \rightarrow \mathrm{R}^{*}>0$ para $\mathrm{n}<6$
b) $\quad \mathrm{R}<0 \rightarrow \mathrm{R}^{*}<0$ para $\mathrm{n}>6$
c) $\quad \mathrm{R}=0 \rightarrow \mathrm{R}^{*}=0$ para $\mathrm{n}=6$.

Demonstração: É conseqüência imediata da expressão 3.2.d.

Proposição 3.3.3. Se $x \rightarrow \partial M_{h}$, então
a) Para $\mathrm{n}<4, \operatorname{Ric}^{*}(\mathrm{x})(\psi, \psi) \rightarrow \infty$ para todo $\psi$ não nulo.
b) Para $\mathrm{n}=4, \operatorname{Ric}^{*}(\mathrm{x})(\psi, \psi) \rightarrow \infty$ para todo $\psi$ não ortogonal ao grad $\mathrm{V}$, isto é, $\operatorname{dV}(\psi)$ não nulo.
c) Para $\mathrm{n}>4$, 
$\left.\mathrm{c}_{1}\right) \operatorname{Ric}^{*}(\mathrm{x})(\psi, \psi) \rightarrow \infty$ se $\psi$ está dentro do cone definido pelo ângulo $\theta=\cos ^{-1}\left(\frac{1}{\sqrt{3}}\right)$ do gradV como a direção de eixo.

$\left.\mathrm{c}_{2}\right) \operatorname{Ric}^{*}(\mathrm{x})(\psi, \psi) \rightarrow-\infty$ se $\psi$ está do lado de fora do cone definido pelo ângulo $\beta=\cos ^{-1}\left(\frac{1}{2}\right)$ do gradV. (obs. em ambos os casos $\psi$ não é ortogonal ao $\operatorname{gradV})$.

Demonstração:

$$
\begin{aligned}
\operatorname{RiC}^{*}(X, Y)= & \operatorname{RiC}(X, Y)+\frac{1}{e^{4 \rho}}[2(h-V)[(n-2) \operatorname{Hess} V(X, Y)+ \\
& +\Delta V\langle X, Y>]+3(n-2) d V(X) d V(Y)+ \\
& \left.-(n-4) N_{v^{2}}\langle X, Y\rangle\right]
\end{aligned}
$$

seja

$$
\begin{aligned}
\Omega= & 2(h-V)[(n-2) \text { Hess } V(\psi, \psi) \Delta V\langle\psi, \psi\rangle]+ \\
& +3(n-2) d V(\psi) d V(\psi)-(n-4) N_{v^{2}}\langle\psi, \psi\rangle
\end{aligned}
$$

Sobre o bordo de $\mathrm{M}_{\mathrm{h}}$,

$$
\Omega=3(n-2) d V(\psi) d V(\psi)-(n-4) N_{v^{2}}\langle\psi, \psi\rangle
$$

e portanto, com os mesmos argumentos da demonstração do teorema 3.3.1, seguem (a) e (b).

Para demonstrar (c), note que se $\psi$ é um vetor unitário qualquer não ortogonal a gradV e $\alpha$ o ângulo entre gradV e $\psi$, então

$$
\cos \alpha=\frac{\langle\psi, \operatorname{grad} V\rangle}{\|g r a d V\|}
$$


Sobre o bordo de $\mathrm{M}_{\mathrm{h}}$,

$$
\begin{aligned}
\Omega & =3(n-2) N_{v^{2} \operatorname{Cos}^{2} \theta-(n-4) N_{v^{2}}} \\
& =\left(3(n-2) \cos ^{2} \theta-(n-4)\right) N_{v^{2}}
\end{aligned}
$$

Assim para $n>4$, e sobre o bordo

$$
\begin{aligned}
& \Omega>0 \rightarrow 3(n-2) \cos ^{2} \theta>(n-4) \rightarrow \cos ^{2} \theta>\frac{n-4}{3(n-2)} \\
& \Omega<0 \rightarrow 3(n-2) \cos ^{2} \theta<(n-4) \rightarrow \cos ^{2} \theta<\frac{n-4}{3(n-2)} .
\end{aligned}
$$

Usando o fato de que

$$
\frac{1}{9} \leq \frac{n-4}{3(n-2)}<\frac{1}{3}, n \geq 5
$$

temos

Se $\cos ^{2} \theta \geq \frac{1}{3}$ então $\Omega>0$ e se $\cos ^{2} \theta<\frac{1}{9}$ então $\Omega<0$

Usando a continuidade das funções envolvidas em $\Omega$, segue que em uma vizinhança do bordo $\partial \mathrm{M}_{\mathrm{h}}$,

$$
\operatorname{RiC}^{*}(\xi, \xi) \rightarrow \infty \text { quando } x \rightarrow \partial M_{h} \text {, se } \cos ^{2} \theta \geq \frac{1}{3}
$$

$\mathrm{e}$

$$
\operatorname{Ric} *(\xi, \xi) \rightarrow-\infty \text { quando } x \rightarrow \partial M_{h}, \text { se } \cos ^{2} \theta<\frac{1}{4}
$$

Observação. Se $\xi$ for ortogonal para gradV, $\Omega$ é nulo sobre o bordo de $M_{h}$ e o 
tensor Ric ${ }^{*}$ fica dependendo do sinal de $(n-2) \operatorname{Hess} V(\xi, \xi)+\Delta V\langle\xi, \xi\rangle$ próximo do bordo.

Proposição 3.3.4. Se $x \rightarrow \partial M_{h}$, então

a) $K^{*} \rightarrow \infty$ se $\pi$ contém um vetor $\xi$ interior ao cone definido peloângulo $\cos ^{-1}\left(\frac{1}{\sqrt{3}}\right)$ do gradV como eixo.

b) Supondo $\pi$ contendo um vetor ortogonal ao gradV, então $K^{*}(\pi) \rightarrow-\infty$ se o plano $\pi$ esta estritamente do lado externo de algum cone como em (a). E se $(\operatorname{HessV}(\xi, \xi)+\operatorname{HessV}(\eta, \eta)) \neq 0$ sobre $\partial \mathrm{M}_{\mathrm{h}}$, então $\left|K^{*}(\pi)\right| \rightarrow \infty$ se o plano $\pi$ toca o cone.

Observação. Em particular, quando $x \rightarrow \partial M_{h^{\prime}}$

$$
\begin{aligned}
& \mathrm{K}^{*}(\pi) \rightarrow \infty \text { se } \pi \text { contém gradV } \\
& \mathrm{K}^{*}(\pi) \rightarrow-\infty \text { se } \pi \text { é ortogonal ao gradV. }
\end{aligned}
$$

Demonstração: Sejam $\pi$ o plano gerado por $(\xi, \eta)$ ortogonais relativo à métrica $<,>$, e $(\alpha, \beta)$ tais que

$$
\begin{aligned}
& \cos \alpha=\frac{\langle\xi, \text { gradV }\rangle}{\mid \text { gradV } \mid} \\
& \cos \beta=\frac{\langle\eta, \text { gradV }\rangle}{\mid \text { gradV } \mid}
\end{aligned}
$$

por 3.2.b,

$$
\begin{aligned}
C(X, Y) & =\frac{n-2}{e^{4 \rho}}[2(h-V) \operatorname{HessV}(X, Y)+ \\
& \left.+3 d V(X) d V(Y)-\frac{N_{V^{2}}}{2}\langle X, Y\rangle\right] .
\end{aligned}
$$

Assim, 


$$
\begin{aligned}
K^{*}(\pi)= & \frac{1}{e^{2 \rho}}\left[K(\pi)+\frac{1}{\mathrm{n}-2}(C(\xi, \xi)+C(\eta, \eta)]\right. \\
= & \frac{1}{e^{2 \rho}}\left[K(\pi)+\frac{1}{e^{4 \rho}}\left[2(h-V) \operatorname{HessV}(\xi, \xi)+3 \operatorname{dV}^{2}(\xi)+\right.\right. \\
& \left.-\frac{N_{v^{2}}}{2}|\xi|^{2}+2(h-V) \operatorname{HessV}(\eta, \eta)+3 d V^{2}(\eta)-\frac{N_{v^{2}}}{2}|\eta|^{2}\right]
\end{aligned}
$$

ou,

$$
\begin{aligned}
\mathrm{K}^{*}(\pi)= & \frac{1}{\mathrm{e}^{2 \rho}}\left[\mathrm{K}(\pi)+\frac{1}{\mathrm{e}^{4 \rho}}(2(\mathrm{~h}-\mathrm{V})[\operatorname{HessV}(\xi, \xi)+\right. \\
& \left.\left.+\operatorname{Hess}(\eta, \eta)+3\left(\mathrm{dV}^{2}(\xi)+\mathrm{dV}^{2}(\eta)\right)-\mathrm{N}_{\mathrm{v}^{2}}\right)\right] .
\end{aligned}
$$

Seja

$$
\begin{aligned}
\Omega= & 2(\mathrm{~h}-\mathrm{V})[\operatorname{HessV}(\xi, \xi)+\operatorname{HessV}(\eta, \eta)+ \\
& \left.+3\left(\mathrm{dV}^{2}(\xi)+\mathrm{dV}^{2}(\eta)\right)-\mathrm{N}_{\mathrm{v}^{2}}\right]
\end{aligned}
$$

sobre o bordo de $\mathrm{M}_{\mathrm{h}}$

$$
\begin{aligned}
\Omega & =3 \cos ^{2} \alpha|\operatorname{gradV}|^{2}+3 \cos ^{2} \eta|\operatorname{gradV}|^{2}-\mathrm{N}_{\mathrm{v}^{2}} \\
& =\left(3 \cos ^{2} \alpha+3 \cos ^{2} \beta-1\right) \mathrm{N}_{\mathrm{v}^{2}} .
\end{aligned}
$$

Logo,

$$
\cos ^{2} \alpha>\frac{1}{3} \rightarrow \Omega>0
$$

e portanto segue (a) usando a expressão de $\mathrm{K}^{*}$.

Para provar (b), suponha $\xi$ ortogonal ao gradV. Então

$$
\Omega=\left(3 \cos ^{2} \beta-1\right) N_{\mathrm{V}^{2}} \text { sobre o bordo de } \mathrm{M}_{\mathrm{h}} \text {, e portanto }
$$




$$
\cos ^{2} \beta<\frac{1}{3} \rightarrow \Omega>0
$$

o que implica pela expressão de $\mathrm{K}^{*}$ que

$$
K^{*}(\pi) \rightarrow-\infty \text { quando } x \rightarrow \partial M_{h} \text {. }
$$

No caso que $\cos ^{2} \beta=\frac{1}{3}$

segue que

$$
\Omega=2(h-V)(\operatorname{Hess} V(\xi, \xi)+\operatorname{Hess}(\eta, \eta)) .
$$

Assim,

$$
\begin{gathered}
\mathrm{K}^{*}(\pi) \rightarrow \operatorname{sgn}(\operatorname{HessV}(\xi, \xi)+\operatorname{HessV}(\eta, \eta)) \infty \\
\text { quando } \mathrm{x} \rightarrow \partial \mathrm{M}_{\mathrm{h}} .
\end{gathered}
$$




\section{\# 3.4. Funções Curvatura de um Sistema}

\section{Mecânico Simples.}

Na seção 3.1 foi definido um operador auto-adjunto que dá informações do tensor de Riemann mecânico. Já na seção 3.2 este operador aparece de forma explícita nas expressões das curvaturas mecânicas. Agora serão utilizados seus auto-valores para obter os sinal da curvatura h-mecânica Seccional. Para isto, serão definidas funções, também chamadas de funções curvaturas, usando os auto-valores do operador auto-adjunto $C^{*}$, como começa a ser feito a seguir:

Sobre uma carta $(U, \phi)$ em $U \subset M$, seja $C^{*}{ }_{U}$ a representação local de $C^{*}$.

$$
\mathrm{C}_{\mathrm{U}}^{*}(\mathrm{x}): \mathrm{E} \rightarrow \mathrm{E} .
$$

Em outra carta $(\mathrm{V}, \psi), \mathrm{C}_{\mathrm{v}}^{*}(\mathrm{x}): \mathrm{E} \rightarrow \mathrm{E}$.

Se $\rho=\psi_{0} \phi^{-1}: \psi(\mathrm{U} \cap \mathrm{V}) \rightarrow \psi(\mathrm{U} \cap \mathrm{V})$ é a mudança de coordenadas, então o seguinte diagrama comuta:

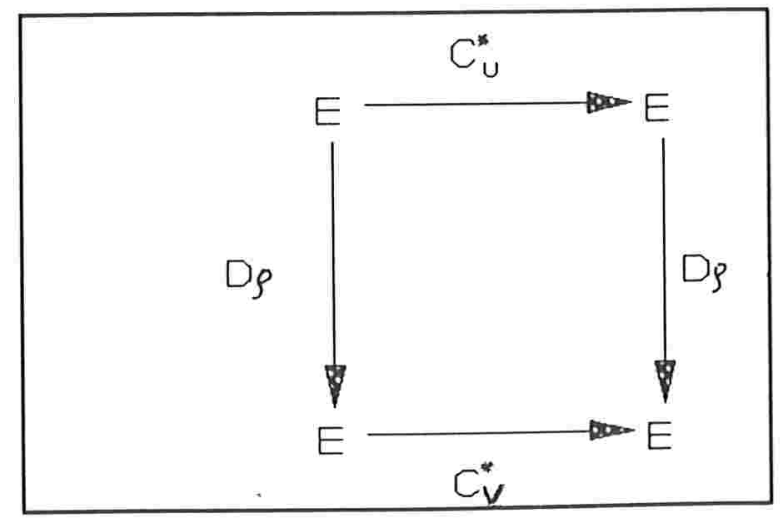


ou seja, $\mathrm{D} \rho(\mathrm{x}) \mathrm{C}^{*}{ }_{\mathrm{U}}(\mathrm{x}) \mathrm{D} \rho(\mathrm{x})^{-1}=\mathrm{C}_{\mathrm{v}}^{*}(\mathrm{x})$.

Definição 3.4.1. Se $\Psi$ é um polinômio em $M(\mathrm{n}, \mathrm{R})$, Álgebra de Lie de matrizes nxn sobre $\mathrm{R}$, dizemos que $\Psi$ é invariante se para cada $\mathrm{X}$ em $M(\mathrm{n}, \mathrm{R})$, $\Psi(\mathrm{X})=\Psi\left(\mathrm{AXA}^{-1}\right)$ para todo A em $G l(\mathrm{n}, \mathrm{R})$, onde $G l(\mathrm{n}, \mathrm{R})$ é o Grupo de Lie das matrizes nxn inversíveis.

Na Geometria, estes polinômios invariantes são de grande utilidade, principalmente quando existe a necessidade de trabalhar com mudanças de coordenadas, como mostra a proposição a seguir:

Proposição 3.4.2. Para qualquer polinômio invariante $\Psi, \Psi\left(C^{*}(x)\right)$ está definido independentemente de representação de coordenadas e portanto está definido sobre toda variedade mecânica $\mathrm{M}_{\mathrm{h}}$, por $\Psi\left(\mathrm{C}^{*}(\mathrm{p})\right)=\Psi\left(\mathrm{C}_{\mathrm{U}}^{*}(\phi(\mathrm{p}))\right)$.

A demonstração segue trivialmente da expressão

$$
\mathrm{D}_{\rho}(\mathrm{x}) \mathrm{C}_{\mathrm{U}}^{*}(\mathrm{x}) \mathrm{D}_{\rho}(\mathrm{x})^{-1}=\mathrm{C}^{*}{ }_{\mathrm{v}}(\mathrm{x}),
$$

e da definição de polinômio invariante.

Definição 3.4.3. Seja $\mathrm{A}: \mathrm{E} \rightarrow \mathrm{E}$ um operador linear auto-adjunto, com seus respectivos auto-valores $\lambda_{1}, \ldots, \lambda_{n}$. Os polinômios 


$$
P_{i}\left(\lambda_{1}, \ldots, \lambda_{n}\right)=\sum_{s_{1}<\ldots<s_{i}} \lambda_{s_{1}} \ldots \lambda_{s_{i}} \quad i=1, \ldots, n
$$

são chamados de polinômios simétricos elementares do operador A.

Observação: Polinômios simétricos são aqueles invariantes por qualquer permutação de $\left(\lambda_{1}, \ldots, \lambda_{n}\right)$.

Dado um operador linear auto-adjunto A com respectivos auto-valores $\lambda_{1}, \ldots, \lambda_{\mathrm{n}}$, considere o polinômio $\mathrm{Q}(\mathrm{z})$, definido por

$$
\begin{aligned}
Q(z) & =\prod_{i<j j}\left(z-\left(\lambda_{i}+\lambda_{j}\right)\right) \\
& =z^{m}-q_{1}\left(\lambda_{1}, \ldots, \lambda_{n}\right) z^{n-1}+\ldots+(-1)^{m} q_{m}\left(\lambda_{1}, \ldots, \lambda_{m}\right)
\end{aligned}
$$

onde $m=\frac{n !}{(n-2) ! 2}$

Proposição 3.4.4. Para $\mathrm{i}=1, \ldots, \mathrm{m}, \mathrm{q}_{\mathrm{i}}\left(\lambda_{1}, \ldots, \lambda_{\mathrm{n}}\right)$ são polinômios simétricos em $\left(\lambda_{1}, \ldots, \lambda_{n}\right)$. Consequentemente,

$$
\mathrm{q}_{\mathrm{i}}\left(\lambda_{1}, \ldots, \lambda_{\mathrm{n}}\right) \in \mathrm{R}\left[\mathrm{p}_{1}(\lambda), \ldots, \mathrm{p}_{\mathrm{n}}(\lambda)\right] .
$$

A demonstração segue do fato que $\mathrm{Q}(\mathrm{z})$ é invariante sobre qualquer permutação de $\left\{\lambda_{1}, \ldots, \lambda_{n}\right\}$

Definição 3.4.5. Seja A um operador linear auto-adjunto com autovalores $\lambda_{1}, \ldots, \lambda_{\mathrm{n}}$. Os polinômios $\mathrm{q}_{\mathrm{i}}\left(\lambda_{1}, \ldots, \lambda_{\mathrm{n}}\right), \mathrm{i}=1, \ldots, \mathrm{m}$ são chamados $\mathrm{de}$ curvaturas polinomiais simétricas de A. 
Definição 3.4.6. As h-funções curvaturas de um sistema mecânico simples são as funções $Q_{j}: M_{h} \rightarrow R, j=1, \ldots, m$, definidas por

$$
\mathrm{Q}_{\mathrm{j}}(\mathrm{p})=\mathrm{q}_{\mathrm{j}}\left(\mathrm{C}^{*}(\mathrm{p})\right), \mathrm{j}=1, \ldots, \mathrm{m},
$$

onde $\mathrm{q}_{\mathrm{j}}\left(\mathrm{C}^{*}(\mathrm{p})\right)$ são as curvaturas polinomiais simétricas de $\mathrm{C}^{*}(\mathrm{x})$.

A proposição a seguir mostra como as funções curvaturas podem auxiliar na análise dos sinais das curvaturas mecânicas e, portanto, no comportamento das curvas físicas de um Sistema h-Mecânico.

\section{Proposição 3.4.7.}

a) $\operatorname{Se} K(x) \pi \geq 0$ e as funções curvaturas $Q_{i}(x) \geq 0$ para $i=1, \ldots, m$, então

$$
\mathrm{K}^{*}(\mathrm{x}) \pi \geq 0 .
$$

b) $\operatorname{Se} \mathrm{K}(\mathrm{x}) \pi \leq 0 \mathrm{e}$

$$
\begin{aligned}
& \mathrm{Q}_{\mathrm{i}}(\mathrm{x}) \leq 0 \text { para i ímpar } \\
& \mathrm{Q}_{\mathrm{i}}(\mathrm{x}) \geq 0 \text { para i par }
\end{aligned}
$$

então

$$
\mathrm{K}^{*}(\mathrm{x}) \pi \leq 0
$$

c) $\operatorname{Se} \mathrm{K}(\mathrm{x}) \pi=0$, então
a) $\mathrm{K}^{*}(\mathrm{x}) \pi \geq 0$ se, e somente se $\mathrm{Q}_{\mathrm{i}}(\mathrm{x}) \geq 0$
b) $\mathrm{K}^{*}(\mathrm{x}) \pi \leq 0$ se, e somente se $\mathrm{Q}_{\mathrm{i}}(\mathrm{x}) \leq 0$ para $\mathrm{i}$ ímpar e $\mathrm{Q}_{\mathrm{i}}(\mathrm{x}) \geq 0$ 
para i par

Para a demonstração da proposição 3.4 .7 , são necessários os dois lemas seguintes.

Lema 3.4.8. Sejam A um operador linear auto-adjunto sobre um espaço com produto interno real $\mathrm{E}$ de dimensão $\mathrm{n}$ e $\lambda_{1}, \lambda_{2}, \ldots, \lambda_{\mathrm{n}}$ os auto-valores correspondentes aos auto-vetores ortonormais $\mathrm{e}_{1}, \ldots, \mathrm{e}_{\mathrm{n}}$ respectivamente. Então

$$
<\text { Ax, } x>+<\text { Ay, } y>\geq 0(\leq 0)
$$

para todos os pares de vetores ortonormais $\mathrm{x}, \mathrm{y}$ em E se, e somente se

$$
\lambda_{\mathrm{i}}+\lambda_{\mathrm{j}} \geq 0(\leq 0) \text { para todo } \mathrm{i} \neq \mathrm{j} .
$$

Demonstração do Lema 3.4.8.

$(\rightarrow)$ Fazendo $x=e_{i}, y=e_{j}$, temos

$<$ Ax, $x>+<$ Ay, $y>=\lambda_{i}<e_{i}, e_{i}>+\lambda_{j}<e_{j}, e_{j}>=\lambda_{i}+\lambda_{j} \geq 0$.

$(\leftarrow)$ Seja $\lambda_{1}=\min \left[\lambda_{1}, \ldots, \lambda_{n}\right]$. Então $\lambda_{\mathrm{j}} \geq 0$ e $\left|\lambda_{1}\right| \leq \lambda_{j}$ para $\mathrm{j} \geq 2$ desde que $\lambda_{1}+\lambda_{\mathrm{j}} \geq 0$ para $\mathrm{j}>1$.

Sejam $\mathrm{x}$, y dois vetores ortornormais. Escrevemos

$$
\begin{aligned}
& \mathrm{x}=\mathrm{x}_{1} \mathrm{e}_{1}+\theta \\
& \mathrm{y}=\mathrm{y}_{1} \mathrm{e}_{1}+\gamma
\end{aligned}
$$

onde $\mathrm{e}_{1}$ é o auto-vetor relativo a $\lambda_{1}$, e

$$
<\theta, \mathrm{e}_{1}>=<\gamma, \mathrm{e}_{2}>=0
$$


e portanto

$$
<\mathrm{x}, \mathrm{y}\rangle=0=\mathrm{x}_{1} \mathrm{y}_{1}+\langle\theta, \gamma\rangle
$$

Se $x_{1}=y_{1}=0$ e $\theta=\sum_{i=2}^{n} w_{i} e_{i}, \quad \gamma=\sum_{i=1}^{n} z_{i} e_{i}$, então

$$
\begin{aligned}
\langle A x, x\rangle+\langle A y, y\rangle & =\left\langle A x_{1} e_{1}+A \theta, x_{1} e_{1}+\theta\right\rangle+\left\langle A y_{1} e_{1}+A \gamma_{1} y_{1} e_{1}+\gamma\right\rangle \\
& =\sum_{j \geq 2} \lambda_{j}\left(w_{j}^{2}+z_{j}^{2}\right) .
\end{aligned}
$$

$x_{1}^{2}+\|\theta\|=y_{1}^{2}+\|\gamma\|=1$. Suponha $y_{1}$ não nulo, então $x_{1}=-\frac{\langle\theta, \gamma\rangle}{y_{1}}$. Assumindo que $\|x\|^{2}=\|y\|^{2}=1$, segue que $x_{1}^{2}+\|\theta\|^{2}=y_{1}+\|\gamma\|^{2}=1$. Por outro lado,

$$
\begin{aligned}
& x_{1}^{2}=\frac{\langle\theta, \gamma\rangle^{2}}{y_{1}^{2}} \leq \frac{\|\theta\|^{2}\|\gamma\|^{2}}{y_{1}^{2}} \rightarrow x_{1}^{2} y_{1}^{2} \leq\|\theta\|^{2}\|\gamma\|^{2} \rightarrow \\
& x_{1}^{2} y_{1}^{2}=\left(1-\|\theta\|^{2}\right)\left(1-\|\gamma\|^{2}\right) \leq\|\theta\|^{2}\|\gamma\|^{2}
\end{aligned}
$$

logo, $\|\theta\|^{2}+\|\gamma\|^{2} \geq 1 \rightarrow x_{1}^{2}+y_{1}^{2}=2-\left(\|\theta\|^{2}+\|\gamma\|^{2}\right) \leq 1$. Consequentemente:

$$
\begin{aligned}
\langle A \mathrm{x}, \mathrm{x}\rangle+\langle A \mathrm{y}, \mathrm{y}\rangle & =\langle A \theta, \theta\rangle+\langle A \gamma, \gamma\rangle+\lambda_{1} \mathrm{x}_{1}^{2}+\lambda_{1} \mathrm{y}_{1}^{2} \\
& z \lambda\left(\|\theta\|^{2}+\|\gamma\|^{2}\right)+\lambda_{1}\left(\mathrm{x}_{1}^{2}+\mathrm{y}_{1}^{2}\right)
\end{aligned}
$$

onde $\lambda=\min _{2 \leq j \leq n}\left\{\lambda_{j}\right\}, \lambda \geq 0$. Se $\lambda_{1} \geq 0$ então $\langle\mathrm{Ax}, \mathrm{x}\rangle+\langle\mathrm{Ay}, \mathrm{y}\rangle \geq 0$. Se $\lambda_{1}<0$, então $\langle\mathrm{Ax}, \mathrm{x}\rangle+\langle$ Ay, $\mathrm{y}\rangle \geq \lambda+\lambda_{1} \geq 0$.

c.q.d.

Lema 3.4.9. Sejam $\mathrm{q}_{\mathrm{i}}(\mathrm{A})$ as curvaturas polinomiais invariantes de $\mathrm{A}$.

\section{Então}




$$
<\text { Ax, } x>+<\text { Ay, } y>\geq 0
$$

para todo par de vetores ortonormais $\mathrm{x}, \mathrm{y}$ em E se, e somente se

$$
\mathrm{q}_{\mathrm{i}}(\mathrm{A}) \geq 0 \text { para } \mathrm{i}=1, \ldots, \mathrm{m},
$$

$\mathrm{e}$

$$
\begin{gathered}
<\text { Ax, } x>+<\text { Ay, y }>\leq 0 \leftrightarrow \mathrm{q}_{\mathrm{i}}(\mathrm{A}) \leq 0 \text { para i ímpar } \\
\mathrm{q}_{\mathrm{i}}(\mathrm{A}) \geq 0 \text { para i par }
\end{gathered}
$$

Demonstração do Lema 3.4.9. Notemos que $\lambda_{\mathrm{i}}+\lambda_{\mathrm{j}}(\mathrm{i}<\mathrm{j})$ são raízes do

polinômio

$$
Q(x)=\prod_{i<j}\left(x-\left(\lambda_{i}+\lambda_{j}\right)\right) x^{m}-q_{1}(A) x^{m-1}+\ldots+(-1)^{m} G_{m}(A) .
$$

Afirmação 3.4.10. As raízes de $Q(x)$ não são negativas se, e somente se,

$$
\mathrm{q}_{\mathrm{i}}(\mathrm{A}) \geq 0 \text { para } \mathrm{i}=1, \ldots, \mathrm{m} .
$$

Demonstração da afirmação 3.4.10. Sejam $\mathrm{r}_{1}, \ldots, \mathrm{r}_{\mathrm{m}}$ as raízes do polinômio $\mathrm{Q}(\mathrm{x})$. Então

$$
q_{1}(A)=\sum_{i=1}^{m} r_{i}, \quad q_{2}(A)=\sum_{i<j} r_{i} r_{j}, \ldots, q_{m}(A)=r_{1} \ldots r_{m},
$$

$(\rightarrow)$ Se $r_{i} \geq 0$ para $\mathrm{i}=1, \ldots, \mathrm{m}$ então $\mathrm{q}_{\mathrm{i}}(\mathrm{A}) \geq 0$ para todo $\mathrm{i}$.

$(\leftarrow)$ Se $\mathrm{q}_{\mathrm{i}}(\mathrm{A}) \geq 0$ para $\mathrm{i}=1, \ldots, \mathrm{m}$ e existe uma raiz negativa $-\lambda(\lambda>0)$, então

$$
\mathrm{Q}(-\lambda)=(-\lambda)^{\mathrm{m}}-\mathrm{q}_{1}(A)(-\lambda)^{\mathrm{m}-1}+\ldots+(-1)^{\mathrm{m}} \mathrm{q}_{\mathrm{m}}(A)=0
$$

$\log$, 
se $\mathrm{m}$ é par então $\mathrm{Q}(-\lambda)>0$

se m é ímpar então $Q(-\lambda)<0$.

Nos dois casos, contradiz a hipótese de $-\lambda$ ser raiz.

c.q.d.

Afirmação 3.4.11. As raízes de $Q(x)$ não são positivas se, e somente se,

$$
\begin{gathered}
\mathrm{q}_{\mathrm{i}}(\mathrm{A}) \leq 0 \text { para i impar } \\
\mathrm{q}_{\mathrm{i}}(\mathrm{A}) \geq 0 \text { para i par, } \mathrm{i}=1, \ldots, \mathrm{m}
\end{gathered}
$$

Com estas duas afirmações mais o lema 3.4 .8 segue a demonstração da afirmação 3.4.11. Se $r_{i} \leq 0$, então $q_{i}(A) \leq 0$ para $i$ impar e $q_{i}(A) \geq 0$ para $i$ par. Por outro lado, usando o mesmo argumento da demonstração da afirmação 3.5.10, um valor positivo não pode ser uma raiz de $\mathrm{Q}(\mathrm{x})$.

$$
\text { c.q.d. }
$$

A demonstração do lema 3.4.9, segue das duas afirmações mais o lema 3.4.8.

Seja $\pi$ o plano gerado por dois vetores ortonormais $\{\xi, \eta\}$. Pela expressão 3.2.e, segue que

$$
K_{p}^{*}(\pi)=\frac{1}{e^{2 \rho}}\left[k_{p}(\pi)+\frac{1}{(n-2)}\left(\left\langle C^{*}(p) \xi, \xi\right\rangle+\left\langle C^{*}(p) \eta, \eta\right\rangle\right)\right]
$$

A demonstração da proposição 3.4.7 segue dos lemas 3.4 .8 e 3.4.9. 


\section{\# 3.5. Curvatura Mecânica em pontos Críticos}

\section{do Potencial V.}

Os pontos críticos sempre dão importantes informações e por este motivo serão estudados nesta seção.

No que segue, $\mathrm{H}(\mathrm{p})$ representará a $\operatorname{HessV}(\mathrm{p})$ e $\mathrm{H}^{*}(\mathrm{p})$ o operador linear auto-adjunto correspondente para a forma bilinear $\mathrm{H}(\mathrm{p})$.

Proposição 3.5.1. Seja p um ponto crítico do potencial V (isto é, $d V(p)$

$=0)$.

a) Se $\mathrm{K}(\mathrm{p}) \pi \geq 0$ e $\mathrm{q}_{\mathrm{i}}\left(\mathrm{H}^{*}(\mathrm{p})\right)>0, \mathrm{i}=1, \ldots, \mathrm{m}$, então existe uma vizinhança p sobre a qual a Curvatura Seccional Mecânica $K^{*}(\pi)$ é positiva.

b) $\mathrm{Se} \mathrm{K}(\mathrm{p}) \pi \leq 0 \mathrm{e}$

$$
\begin{aligned}
\mathrm{q}_{\mathrm{i}}\left(\mathrm{H}^{*}(\mathrm{p})\right) & >0 \text { para i ímpar } \\
\mathrm{q}_{\mathrm{i}}\left(\mathrm{H}^{*}(\mathrm{p})\right) & <0 \text { para i par }
\end{aligned}
$$

então $\mathrm{K}^{*}(\mathrm{p}) \pi$ é negativa sobre uma vizinhança de $\mathrm{p}$.

Demonstração: Por 3.2.e,

$$
\begin{aligned}
K_{p}^{*} & =\frac{1}{e^{2 p}}\left[K_{p}(\pi)-\frac{1}{n-2}\left[\frac{n-2}{h-V} \operatorname{HessV}(p)(X, Y)\langle X, Y\rangle+\right.\right. \\
& \left.-\frac{n-2}{2(h-V)}\left[\operatorname{HessV}(p)(X, X)|Y|^{2}+\operatorname{HessV}(p)(Y, Y)|X|^{2}\right]\right]
\end{aligned}
$$


Tomando $X=\xi$ e $Y=\eta$ ortornormais,

$$
K^{*}(p) \pi=\frac{1}{e^{2 \rho}}\left[K(p) \pi+\frac{1}{2(h-V)}\left\langle\left\langle H^{*}(p) \xi, \xi\right\rangle+\left\langle H^{*}(p) \eta, \eta\right\rangle\right]\right.
$$

A demonstração segue do fato que nos lemas 3.4 .8 e 3.4 .9 , valem as desigualdades estritas (a demonstração deste fato é idêntica trocando apenas o sinal $\geq(\leq)$ por $>(<))$ e pela continuidade de $\mathrm{K}^{*}(\mathrm{p})$.

Definição 3.5.2. A função índice(p) de uma matriz $\mathrm{H}^{*}(\mathrm{p})$ é definida como o número de auto-valores negativos da matriz.

Corolário 3.5.3. Se o índice(p) $=0$, isto é, $\mathrm{p}$ é um mínimo local do potencial $\mathrm{V}$ e $\mathrm{K}(\mathrm{p}) \geq 0$, então $\mathrm{K}^{*}(\mathrm{p})$ é positivo em uma vizinhança de $\mathrm{p}$.

Demonstração: p é mínimo local se, e somente se $\mathrm{H}^{*}(\mathrm{p})$ é positivo definido e isto implica que $\mathrm{q}_{i}\left(\mathrm{H}^{*}(\mathrm{p})\right)>0$ para $\mathrm{i}=1, \ldots, \mathrm{m}$ e assim a prova segue da proposição 3.5 .1 .

c.q.d.

Corolário 3.5.4. Se índice $(\mathrm{p})=\mathrm{n}$, isto é, $\mathrm{p}$ é um máximo local do potencial $\mathrm{V}$ e $\mathrm{K}(\mathrm{p}) \leq 0$ então $\mathrm{K}^{*}(\mathrm{p})$ é negativo em uma vizinhança 
de $p$.

Demonstração. p é um máximo local se, e somente se, $\mathrm{H}^{*}(\mathrm{p})$ é negativo definido e isto implica que

$$
\begin{gathered}
\mathrm{q}_{\mathrm{i}}\left(\mathrm{H}^{*}(\mathrm{p})\right)>0 \text { para } \mathrm{i} \text { impar } \\
\mathrm{q}_{\mathrm{i}}\left(\mathrm{H}^{*}(\mathrm{p})\right)<0 \text { para i par }
\end{gathered}
$$

e pela proposição 3.5.1 segue a demonstração.

Corolário 3.5.5. Se índice $(p) \geq 2$ e $K(p)=0$ então em uma vizinhança de $\mathrm{p}, \mathrm{K}_{\mathrm{p}}^{*}(\pi)<0$ para o mesmo plano $\pi$.

Demonstração. Índice $(p) \geq 2$ significa que $\mathrm{H}^{*}(\mathrm{p})$ é negativo definido sobre um subespaço de dimensão maior ou igual a 2. Então

$$
\mathrm{K}_{\mathrm{p}}^{*}(\pi)=\frac{1}{\mathrm{e}^{4 \rho}}\left(\left\langle\mathrm{H}_{\xi}^{*}, \xi\right\rangle+\left\langle\mathrm{H}_{\eta}^{*}, \eta\right\rangle\right)<0
$$

para qualquer plano bidimensional neste subespaço. 


\section{CAPÍTULO 4 Exemplos}

\section{\# 41 ก nrohlema de n-nartírulac}

Considere $\mathrm{n}$ partículas de massas $\mathrm{m}_{1}, \ldots, \mathrm{m}_{\mathrm{n}}$, movimentando-se sobre o espaço euclidiano $E^{3}$. As posições das partículas estão representadas pelo vetor $\left(x_{1}, \ldots, x_{n}\right)$, $\mathrm{x}_{\mathrm{i}} \in \mathrm{E}^{3}, \mathrm{i}=1, \ldots, \mathrm{n}$. A energia cinética é dada por:

$$
K(p, v)=\sum_{i=1}^{n} \frac{1}{2} m_{i}\left\langle v_{i}, v_{i}\right\rangle, \quad(p, v) \in\left(E^{3}\right)^{n} \times\left(E^{3}\right)^{n}
$$

e a energia potencial está definida pela aplicação

$$
V\left(x_{1}, \ldots, x_{n}\right)=-\sum_{i<j} \frac{m_{i} m_{j}}{\left\|x_{i}-x_{j}\right\|}
$$

As singularidades da função energia potencial $V, x_{i}=x_{j}$ para $i \neq j$, representam as colisões da i-ésima com a j-ésima partícula.

Considere o seguinte conjunto:

$$
\Upsilon=\left\{\left(x_{1}, \ldots, x_{n}\right) \in\left(E^{3}\right)^{n} ; x_{i}=x_{j} \text { para } i \neq j\right\},
$$

assim, a energia potencial $V$ é uma aplicação diferenciável definida sobre a variedade diferenciável $\mathrm{M}=\left(\mathrm{E}^{3}\right)^{\mathrm{n}}-\Upsilon$. Logo, $(\mathrm{M}, \mathrm{K}, \mathrm{V})$ é um sistema mecânico

simples, com energia cinética $\mathrm{K}: \mathrm{TM} \rightarrow \mathrm{R}$ e energia potencial $\mathrm{V}: \mathrm{M} \rightarrow \mathrm{R}$. A variedade diferenciável $\mathrm{M}$ é o espaço de configuração e o fibrado tangente TM é o espaço de fase do sistema mecânico simples. 


\section{\# 4.2. O problema de força central.}

Este segundo exemplo, além de servir como ilustração de um sistema mecânico simples, será estudado em detalhes, para que se possa aplicar os resultados desenvolvidos no trabalho.

O movimento de duas partículas no espaço, sob a ação da força de atração radial, pode ser reduzido de forma canônica para o movimento de uma única partícula, movendo-se em um plano sob uma força central.

Os espaços $E^{2}, E^{2} \times E^{2}$ contém os espaços de configuração e o de fase, respectivamente. Um ponto $\mathrm{p}$ do espaço de configuração pode ser dado em coordenadas polares $(r, \theta)$. Tomando a origem como o centro da força central, a função energia potencial é uma função diferenciável $\mathrm{V}(\mathrm{r})$ independente de $\theta$.

Seja M uma subvariedade aberta de $\mathrm{E}^{2}$ com as singuraridades do potencial $\mathrm{V}$ removidas (em geral $\mathrm{r}=0$ ). Assumindo que as massas das partículas são iguais a um, a energia cinética é dada por:

$$
K(p, v)=\frac{1}{2} g(v, v), \quad(p, v) \in T M,
$$

onde $\mathrm{v}$ é o vetor velocidade e g é o produto interno euclidiano.

A métrica de Jacobi do sistema mecânico simples $(\mathrm{M}, \mathrm{K}, \mathrm{V})$ relativo para o valor de energia h é dado por

$$
\mathrm{g}_{\mathrm{h}}=2(\mathrm{~h}-\mathrm{V}) \mathrm{g}
$$


Seja $\left\{\frac{\partial}{\partial x}, \frac{\partial}{\partial y}\right\}$ uma base ortonormal de $\mathrm{T}_{\mathrm{p}} \mathbf{M}$, e em coordenadas $(\mathrm{r}, \theta)$,

$$
\left(\begin{array}{ll}
\frac{\partial}{\partial r} & \frac{\partial}{\partial \theta}
\end{array}\right)=\left(\begin{array}{ll}
\frac{\partial}{\partial x} & \frac{\partial}{\partial y}
\end{array}\right)\left(\begin{array}{cc}
\cos \theta & -r \operatorname{sen} \theta \\
\operatorname{sen} \theta & r \cos \theta
\end{array}\right)
$$

e assim:

$$
\begin{aligned}
& \left\langle\frac{\partial}{\partial r}, \frac{\partial}{\partial r}\right\rangle=1 \\
& \left\langle\frac{\partial}{\partial r}, \frac{\partial}{\partial \theta}\right\rangle=0 \\
& \left\langle\frac{\partial}{\partial \theta}, \frac{\partial}{\partial \theta}\right\rangle=r^{2}
\end{aligned}
$$

Um referencial local ortonormal para $T_{p} M_{h}$ em relação à mudança de coordenadas é:

$$
\begin{aligned}
& X_{1}=\frac{1}{f} \frac{\partial}{\partial r} \\
& X_{2}=\frac{1}{f I} \frac{\partial}{\partial \theta}
\end{aligned}
$$

onde $\mathrm{f}^{2}=2(\mathrm{~h}-\mathrm{V})$ e com o correferencial correspondente

$$
\theta^{1}=\operatorname{fdr} \text { e } \theta^{2}=\operatorname{frd} \theta .
$$

Assim,

$$
\begin{aligned}
& d \theta^{1}=d f \wedge d r=f^{\prime} d r \wedge d r=0 \\
& d \theta^{2}=(f r)^{\prime} d r \Lambda d \theta=-\omega_{1}^{2} \wedge \theta^{1}
\end{aligned}
$$

logo, temos a forma de conexão:

$$
\omega=\frac{1}{f^{2} r} r f^{\prime} \theta^{2} .
$$


Diferenciando a última expressão, segue que:

$$
d \omega=\frac{1}{f^{2} r}\left[\frac{1}{f}(r f)^{\prime}\right]^{\prime} \theta^{1} \wedge \theta^{2}
$$

Com as ferramentas acima, podemos demonstrar um importante resultado que caracteriza a geometria de $\mathrm{M}_{\mathrm{h}}$.

Proposição 4.2.1. A curvatura gaussiana mecânica $\mathrm{k}_{\mathrm{h}}$ é dada por:

$$
k_{h}=\frac{1}{4 r(h-V)^{3}}\left[(h-V)\left(r V^{\prime}\right)^{\prime}+r\left(V^{\prime}\right)^{2}\right]
$$

Demonstração: A expressão de d $\omega$ nos dá que

$$
\begin{aligned}
k_{h} & =-\frac{1}{f^{2} r}\left[\left(\frac{1}{f}\right)(r f)^{\prime}\right]^{\prime} \\
& =-\frac{1}{r f^{4}}\left[f^{\prime} f+r f f^{\prime \prime}-r\left(f^{\prime}\right)^{2}\right]
\end{aligned}
$$

substituindo $\mathrm{f}^{2}$ por $2(\mathrm{~h}-\mathrm{V})$ na expressão acima, segue que

$$
\begin{aligned}
k_{h} & =-\frac{1}{8 I(h-V)^{3}}\left[-2(h-V) V^{\prime}-2 r(h-V) V^{\prime \prime}-2 r\left(V^{\prime}\right)^{2}\right] \\
& =\frac{1}{4 r(h-V)^{3}}\left[(h-V)\left[r V^{\prime}\right]^{\prime}+r\left(V^{\prime}\right)^{2}\right]
\end{aligned}
$$

c.q.d.

Proposição 4.2.2. Suponha que h é um valor regular de $V$ e que o bordo 
anular $\partial \mathrm{M}_{\mathrm{h}}=\{\mathrm{x} \in \mathrm{M} ; \mathrm{V}(\mathrm{x})=\mathrm{h}\}$ é diferente do vazio. Então existe uma região anular, que é uma vizinhança do bordo $\partial \mathrm{M}_{\mathrm{h}}$, sobre a qual a curvatura $\mathrm{K}_{\mathrm{h}}$ é positiva.

Demonstração: Pela continuidade das funções (rV')', $r\left(V^{\prime}\right)^{2}$, e pelo fato que $(h-V) \rightarrow 0$ quando $x \rightarrow \partial M_{h}$, existe uma região compacta anular tal que (h-V)(rV')' $+\mathrm{r}\left(\mathrm{V}^{\prime}\right)^{2}>0$. Como $(\mathrm{h}-\mathrm{V})>0$, segue que $\mathrm{k}_{\mathrm{h}}$ é positivo nesta região anular compacta, completando assim a demonstração.

Observe que $k_{h} \rightarrow \infty$ quando $x \rightarrow \partial M_{h}$.

Os dois próximos resultados dão informações da geometria da variedade hmecânica em uma vizinhança de um ponto crítico do potencial V.

Proposição 4.2.3. Se o potencial V tem um máximo local (um mínimo local) em $r_{0}$, então $k_{h}(r)>0\left(k_{h}(r)<0\right)$ em uma região anular definida por $\left|r-r_{o}\right|<$ $\delta$ para algum $\delta>0$.

Demonstração: Se $r_{o}$ é um ponto crítico de $V$ então $V^{\prime}\left(r_{0}\right)=0$ e portanto

$$
k_{h}\left(r_{0}\right)=\frac{V^{\prime \prime}\left(r_{0}\right)}{4\left(h-V\left(r_{0}\right)\right)^{2}}
$$

logo se $\mathrm{r}_{\mathrm{o}}$ é um ponto de máximo local (mínimo local), $\mathrm{k}_{\mathrm{h}}\left(\mathrm{r}_{\mathrm{o}}\right)<0\left(\mathrm{k}_{\mathrm{h}}\left(\mathrm{r}_{\mathrm{o}}\right)>0\right)$. Pela continuidade de $\mathrm{k}_{\mathrm{h}}$, existe $\delta>0$ tal que $\mathrm{k}_{\mathrm{h}}<0\left(\mathrm{k}_{\mathrm{h}}>0\right)$ no anel 


$$
\mathrm{A}_{\delta}=\left\{\mathrm{r} \in \mathrm{R}^{+} \text {tal que }\left|\mathrm{r}-\mathrm{r}_{\mathrm{o}}\right|<\delta\right\} .
$$

Proposição 4.2.4. Se V" $\left(r_{0}\right)=0$ e $r_{o}$ for um ponto crítico do potencial V, então $\mathrm{k}_{\mathrm{h}}\left(\mathrm{r}_{\mathrm{o}}\right)=0$.

A demonstração segue da própria expressão de $\mathrm{k}_{\mathrm{h}}$.

Já que a curvatura $k_{h}$ depende apenas de $r$, pode-se dividir $M_{h}$ em bandas concêntricas de curvatura positiva, negativa, separadas por curvas de curvatura nula.

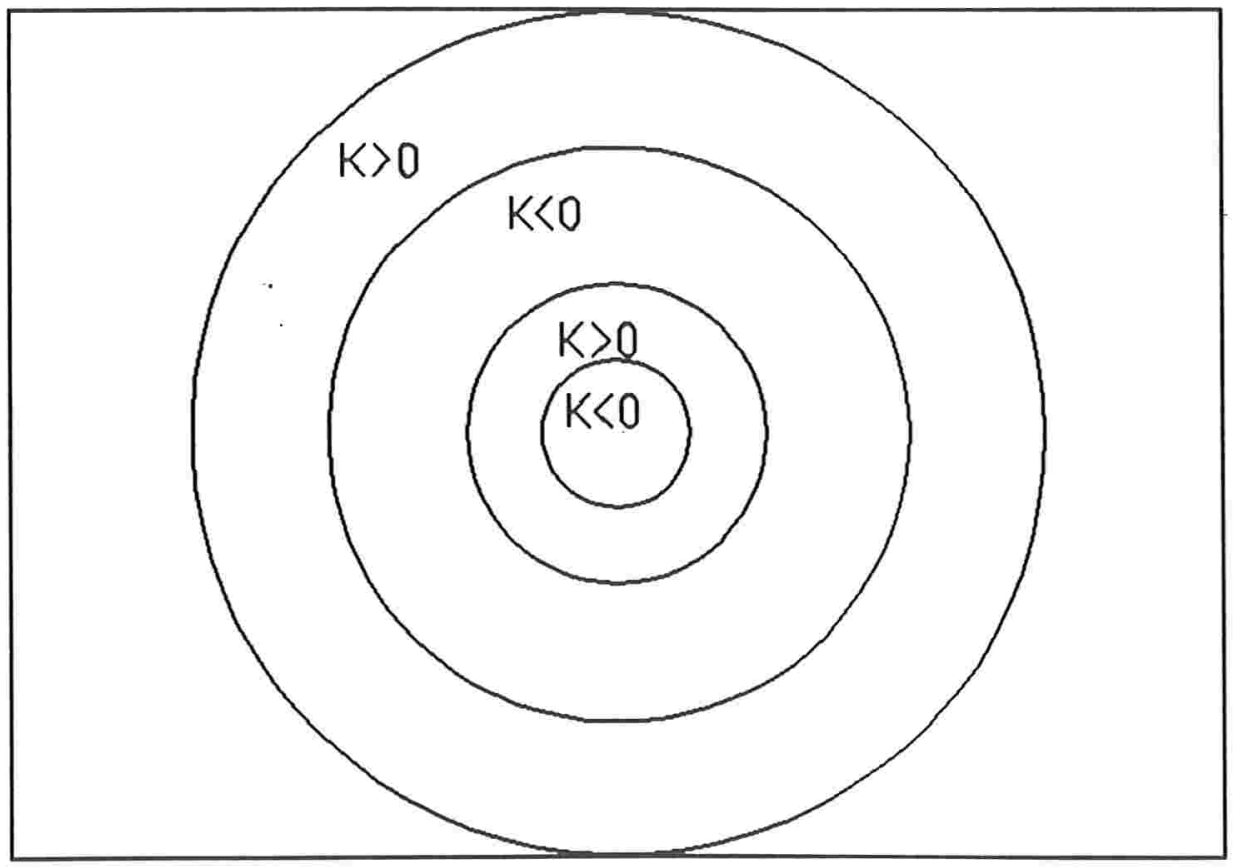

figura 4.2.1 


\section{\# 4.3. O problema de Kepler com o potencial}

\section{Newtoniano}

Um dos problemas mais comuns, quando trabalha-se com o problema de força central é o caso particular Newtoniano, que será estudado a seguir.

Para o movimento de duas partículas sob a ação da força gravitacional Newtoniana, a energia potencial do problema de força central é dada por:

$$
V(x)=-\frac{1}{\|x\|} \text {. }
$$

Desta forma, fica caracterizado a variedade h-mecânica para qualquer valor de energia $h$. Escolhendo as massas e a constante gravitacional iguais a 1 e usando a expressão 4.2.a, segue que:

$$
\begin{aligned}
k_{h} & =\frac{1}{4 r\left(h+\frac{1}{r}\right)^{3}}\left[\left(h+\frac{1}{r}\right)\left(-\frac{1}{r^{2}}\right)+r\left(\frac{1}{r^{2}}\right)^{2}\right. \\
& =-\frac{h}{4(r h+1)}, \quad r=\|x\|
\end{aligned}
$$

Usando o fato que $\mathrm{h}-\mathrm{V}>\mathrm{O}$, encontram-se as seguintes relações:

$$
\begin{aligned}
& h>0 \rightarrow k_{h}(I)<0 \\
& h=0 \rightarrow k_{h}(r)=0 \\
& h<0 \rightarrow k_{h}(I)>0
\end{aligned}
$$

Se $\mathrm{h}$ for positivo, as órbitas são hiperbólicas, no caso em que h é negativo, as órbitas são elípticas, e serão parabólicas quando h for nulo. 
Se $\mathrm{h}<0$ então $4 \mathrm{k}_{\mathrm{h}}>|\mathrm{h}|$, pois caso contrário

$$
\begin{gathered}
\frac{|h|}{4(r h+1)} \leq \frac{1}{4}|h| \\
\frac{1}{r h+1} \leq 1 \\
0 \leq h
\end{gathered}
$$

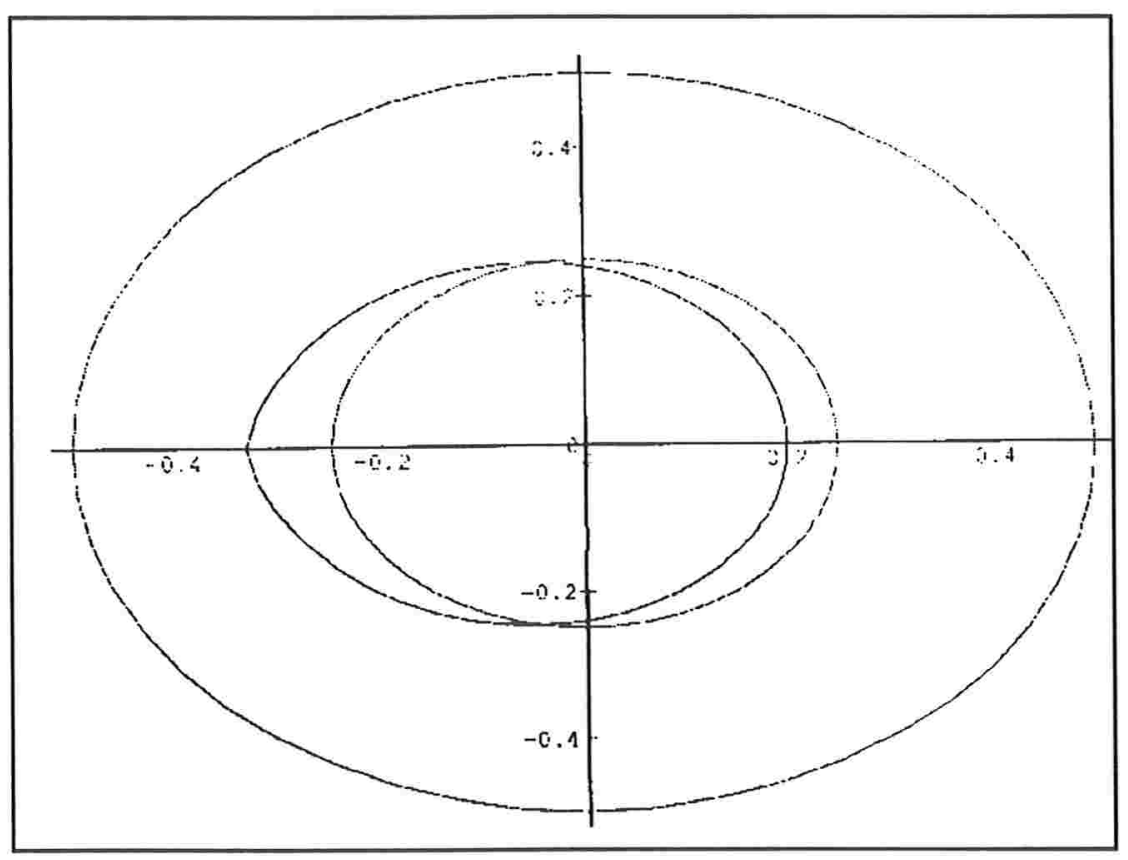

geodésicas com o valor de energia h negativa.

figura 4.3.1. 


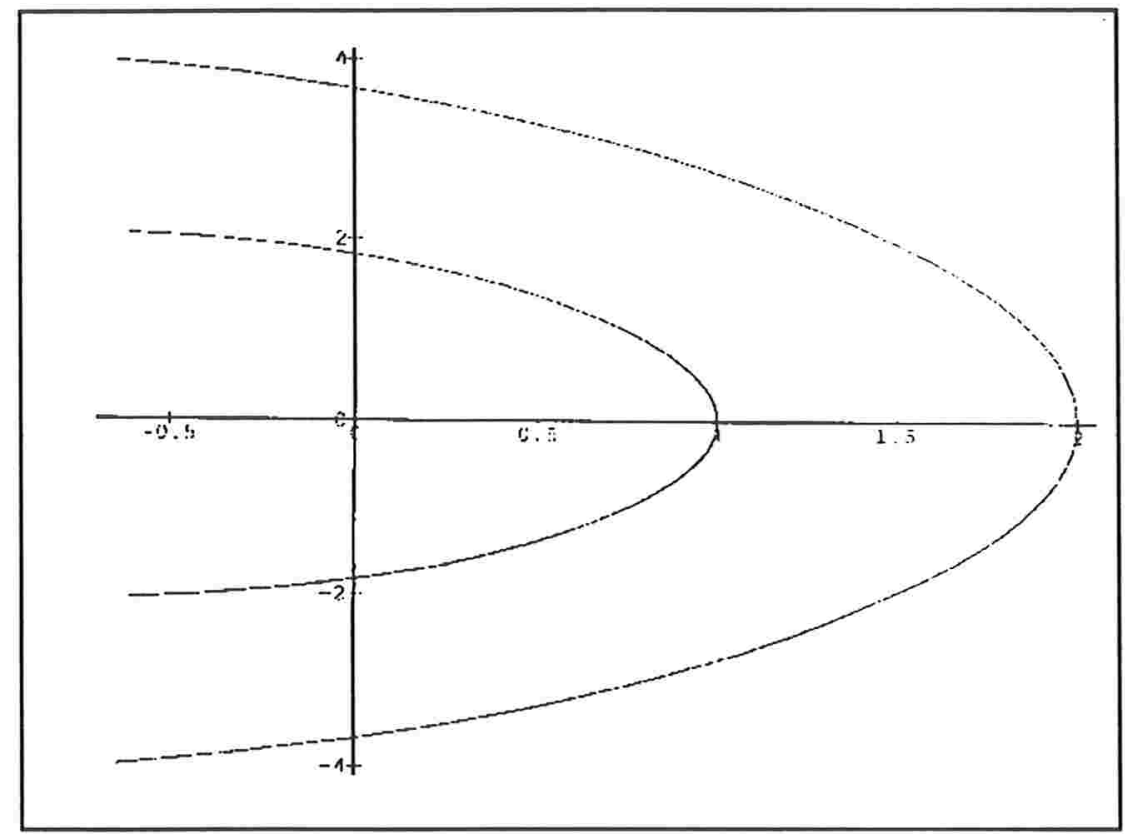

geodésicas com o

valor de energia $h$

nula.

figura 4.3.2. 


\section{\# 4.4. O problema de três partículas}

Considere três partículas com massas $\mathrm{m}_{1}, \mathrm{~m}_{2}, \mathrm{~m}_{3}$, movendo-se no espaço Euclidiano $\mathrm{E}^{3}$. As posições das partículas são dadas pelo vetor $\left(\mathrm{x}_{1}, \mathrm{x}_{2}, \mathrm{x}_{3}\right)$, onde cada $x_{i} \in E^{3}$. Assim, o espaço de configuração está contido em $\left(E^{3}\right)^{3}$. A energia cinética é dada por:

$$
k(p, v)=\sum_{i=1}^{3} \frac{1}{2} m_{i}\left\langle v_{i}, v_{i}\right\rangle \quad(p, v) \in\left(E^{3}\right)^{3} \times\left(E^{3}\right)^{3}
$$

As três partículas movem-se sob a ação do potencial Newtoniano

$$
V\left(x_{1}, x_{2}, x_{3}\right)=-\sum_{i<j} \frac{m_{i} m_{j}}{\left\|x_{i}-x_{j}\right\|} .
$$

Excluindo as posições colisões representadas por:

$$
\Upsilon=\left\{\left(\mathrm{x}_{1}, \mathrm{x}_{2}, \mathrm{x}_{3}\right) \in\left(\mathrm{E}^{3}\right)^{3} ; \mathrm{x}_{\mathrm{i}}=\mathrm{x}_{\mathrm{j}}, \text { para algum } \mathrm{i} \neq \mathrm{j}\right\},
$$

obtém-se que $V$ é uma função diferenciável sobre $\left(E^{3}\right)^{3}-\Upsilon$

Vamos assumir que o centro de massa do sistema está fixado. Isto pode ser feito porque o sistema é invariante por translação. Assim, escolhendo o centro de massas como origem do referencial, nós podemos trabalhar sobre o espaço de configuração $\mathrm{M}$ definido por

$$
M=\left\{\left(x_{1}, x_{2}, x_{3}\right) \in\left(E^{3}\right)^{3} ; \sum_{i=1}^{3} m_{i} x_{i}=0\right\} \backslash \Upsilon
$$

É conveniente introduzir as coordenadas: 


$$
x=x_{2}-x_{1}
$$

4.4.a

$$
y=x_{3}-\frac{m_{1} x_{1}+m_{2} x_{2}}{m_{1}+m_{2}}=\frac{\sigma}{\mu} x_{3}
$$

onde

$$
\begin{aligned}
& \boldsymbol{\sigma}=m_{1}+m_{2}+m_{3} \\
& \mu=m_{1}+m_{2}
\end{aligned}
$$

A energia potencial em função das novas variáveis fica:

$$
V(x, y)=-\frac{m_{1} m_{2}}{\|x\|}-\frac{m_{1} m_{3}}{\|\alpha x+y\|}-\frac{m_{2} m_{3}}{\|\beta x-y\|}
$$

onde

$$
\begin{aligned}
& \alpha=\frac{m_{2}}{\mu} \\
& \beta=\frac{m_{1}}{\mu}
\end{aligned}
$$

Para obter a expressão da energia cinética em função das novas variáveis, usamos 4.4.a, obtendo:

4.4.b. $y=\left(-\frac{m_{1}}{m_{2}}-\frac{m_{1}}{m_{1}+m_{2}}\right) x_{1}+\left(-\frac{m_{2}}{m_{1}+m_{2}}-\frac{m_{2}}{m_{3}}\right) x_{1}+\left(-\frac{m_{2}}{m_{1}+m_{2}}-\frac{m_{2}}{m_{3}}\right)$;

ou seja
4.4.c.

$$
x_{1}=\frac{-m_{3}}{m_{1}+m_{2}+m_{3}}\left[y+\left(\frac{m_{2}}{m_{1}+m_{2}}+\frac{m_{2}}{m_{3}}\right) x\right]
$$

e 
4.4.d.

$$
x_{2}=\frac{-m_{3}}{m_{1}+m_{2}+m_{3}}\left[y-\left(\frac{m_{1}}{m_{3}}+\frac{m_{1}}{m_{1}+m_{2}}\right) x\right]
$$

4.4.e.

$$
x_{3}=\frac{m_{1}+m_{2}}{m_{1}+m_{2}+m_{3}} y
$$

Derivando as expressões 4.4.c, 4.4.d, 4.4.e,

$$
\begin{aligned}
& \dot{x}_{1}=\frac{-m_{3}}{m_{1}+m_{2}+m_{3}}\left(\frac{m_{2}}{m_{1}+m_{2}}+\frac{m_{2}}{m_{3}}\right) \dot{x}-\frac{m_{3}}{m_{1}+m_{2}+m_{3}} \dot{y} \\
& \dot{x}_{2}=\frac{m_{3}}{m_{1}+m_{2}+m_{3}}\left(\frac{m_{1}}{m_{3}}+\frac{m_{1}}{m_{1}+m_{2}}\right) \dot{x}-\frac{m_{3}}{m_{1}+m_{2}+m_{3}} \dot{y} \\
& \dot{x}_{3}=\frac{m_{1}+m_{2}}{m_{1}+m_{2}+m_{3}} \dot{y}
\end{aligned}
$$

Logo, a energia cinética é dada por:

$$
\begin{aligned}
K(\dot{x}, \dot{y}) & =\frac{1}{2} \sum_{i=1}^{3} m_{i}\left\langle v_{i}, v_{i}\right\rangle= \\
& =\frac{1}{2}\left[\frac{m_{1} m_{3}^{2}}{\left(m_{1}+m_{2}+m_{3}\right)^{2}}\left(\frac{m_{2}}{m_{1}+m_{2}}+\frac{m_{2}}{m_{3}}\right)^{2}+\frac{m_{2} m_{3}^{2}}{\left(m_{1}+m_{2}+m_{3}\right)^{2}}\left(\frac{m_{1}}{m_{1}+m_{2}}+\frac{m_{1}}{m_{3}}\right)^{2}\right]\langle\dot{x}, \\
& +\frac{1}{2}\left[\frac{m_{1} m_{3}^{2}}{\left(m_{1}+m_{2}+m_{3}\right)^{2}}+\frac{m_{2} m_{3}^{2}}{\left(m_{1}+m_{2}+m_{3}\right)^{2}}+\frac{m_{3}\left(m_{1}+m_{2}\right)^{2}}{\left(m_{1}+m_{2}+m_{3}\right)^{2}}\right]\langle\dot{y}, \dot{y}\rangle \\
& =\frac{1}{2}\left[\frac{m_{1} m_{2}}{m_{1}+m_{2}}\langle\dot{x}, \dot{x}\rangle+\frac{m_{3}\left(m_{1}+m_{2}\right)}{m_{1}+m_{2}+m_{3}}\langle\dot{y}, \dot{y}\rangle\right]
\end{aligned}
$$

ou ainda,

$$
K(\dot{x}, \dot{y})=\frac{1}{2}\left[g_{1}\langle\dot{x}, \dot{x}\rangle+g_{2}\langle\dot{y}, \dot{y}\rangle\right]
$$

onde 


$$
\begin{aligned}
& g_{1}=\frac{m_{1} m_{2}}{\mu} \\
& g_{2}=\frac{m_{3} \mu}{\sigma}
\end{aligned}
$$

Da expressão energia cinética, segue que

4.4.f.

$$
\left(g_{i j}\right)=\left(\begin{array}{cccccc}
g_{1} & 0 & 0 & 0 & 0 & 0 \\
0 & g_{1} & 0 & 0 & 0 & 0 \\
0 & 0 & g_{1} & 0 & 0 & 0 \\
0 & 0 & 0 & g_{2} & 0 & 0 \\
0 & 0 & 0 & 0 & g_{2} & 0 \\
0 & 0 & 0 & 0 & 0 & g_{2}
\end{array}\right)
$$

e portanto os símbolos de Christoffel são todos nulos e como consequência, segue um importante resultado:

Proposição 4.4.1. A variedade diferenciável $M$ é rasa, isto é, o tensor curvatura de Riemann é nulo.

Para expressar as curvaturas da variedade h-mecânica, são usados a norma $\left(N_{V^{2}}\right)$ e o laplaciano da função energia potencial V.

As coordenadas $\mathrm{x}, \mathrm{y}$ são da forma $(\mathrm{x}, \mathrm{y})=\left(\mathrm{x}^{1}, \mathrm{x}^{2}, \mathrm{x}^{3}, \mathrm{y}^{1}, \mathrm{y}^{2}, \mathrm{y}^{3}\right)$ e as derivadas parciais da função energia potencial V são dadas por: 


$$
\begin{aligned}
& \frac{\partial V}{\partial x^{i}}=\frac{m_{1} m_{2} x^{i}}{\|x\|^{3}}+\frac{m_{1} m_{3} \alpha\left(\alpha x^{i}+y^{i}\right)}{\|\alpha x+y\|^{3}}+\frac{m_{2} m_{3} \beta\left(\beta x^{i}-y^{i}\right)}{\|\beta x-y\|^{3}} \\
& \frac{\partial V}{\partial y^{j}}=\frac{m_{1} m_{3}\left(\alpha x^{i}+y^{i}\right)}{\|\alpha x+y\|^{3}}-\frac{m_{2} m_{3}\left(\beta x^{i}-y^{i}\right)}{\|\beta x-y\|^{3}}
\end{aligned}
$$

Por definição,

$$
N_{V^{2}}=\langle d V, d V\rangle=\sum_{m} V^{m} V_{m}=\sum_{m, i} g^{m i} V_{i} V_{m}
$$

e assim

$$
\begin{aligned}
N_{v^{2}} & =\frac{m_{1} m_{2}\left(m_{1}+m_{2}\right)}{\|x\|^{4}}+\frac{m_{1} m_{2} m_{3}^{2}}{m_{1}+m_{2}} \frac{1}{\|\alpha x+y\|^{4}}+\frac{m_{1} m_{2} m_{3}^{2}}{m_{1}+m_{2}} \frac{1}{\|\beta x-y\|^{4}}+ \\
& +2 m_{1} m_{2} m_{3} \frac{x}{\|x\|^{3}} \frac{\beta x-y}{\|\beta x-y\|^{3}}+\frac{2 m_{1} m_{2} m_{3} x}{\|x\|^{3}} \frac{\alpha x+y}{\|\alpha x+y\|^{3}}+ \\
& +\frac{2 m_{1} m_{2} m_{3}^{2}}{m_{1}+m_{2}} \frac{\alpha x+y}{\|\alpha x+y\|^{3}} \frac{\beta x-y}{\|\beta x-y\|^{3}}+\frac{m_{1}+m_{2}+m_{3}}{m_{1}+m_{2}} \frac{m_{1}^{2} m_{3}}{\|\alpha x+y\|^{4}}+ \\
& +\frac{m_{1}+m_{2}+m_{3}}{m_{1}+m_{2}} \frac{m_{3} m_{2}^{2}}{\|\beta x-y\|^{4}}-2 \frac{m_{1}+m_{2}+m_{3}}{m_{1}+m_{2}} m_{1} m_{2} m_{3} \frac{\alpha x+y}{\|\alpha x+y\|^{3}} \frac{\beta x-y}{\|\beta x-y\|^{3}}
\end{aligned}
$$

ou ainda

$$
\begin{aligned}
N_{V^{2}}= & \frac{m_{1} m_{2}\left(m_{1}+m_{2}\right)}{\|x\|^{4}}+\frac{m_{2} m_{3}\left(m_{2}+m_{3}\right)}{\|\beta x-y\|^{4}}+\frac{m_{1} m_{3}\left(m_{1}+m_{3}\right)}{\|\alpha x+y\|^{4}} \\
& +\frac{2 m_{1} m_{2} m_{3} x}{\|x\|^{3}}\left[\frac{\alpha x+y}{\|\alpha x+y\|^{3}}+\frac{\beta x-y}{\|\beta x-y\|^{3}}\right] \\
& -\frac{2 m_{1} m_{2} m_{3}(\alpha x+y)}{\|\alpha x+y\|^{3}} \frac{\beta x-y}{\|\beta x-y\|^{3}}
\end{aligned}
$$

O Laplaciano da energia potencial V é dado pela proposição a seguir: 
Proposição 4.4.2. Para o problema das três partículas com o potencial Newtoniano em $\mathrm{E}^{3}$, o Laplaciano de $\mathrm{V}$ é nulo.

Demonstração: Por definição,

$$
\begin{aligned}
\Delta V & =\operatorname{trHess} V=\operatorname{tr}\left(\frac{\partial^{2} V}{\partial x^{i} \partial x^{j}}-\sum_{m} V_{m} \Gamma_{j i}^{m}\right) \\
& =\sum_{i, j}\left(g^{i j} \frac{\partial^{2} V}{\partial x^{i} \partial x^{j}}\right)
\end{aligned}
$$

onde

$$
\begin{aligned}
\frac{\partial^{2} V}{\partial x^{i^{2}}} & =\frac{m_{1} m_{2}}{\|x\|^{3}}-\frac{3 m_{1} m_{2} x^{i^{2}}}{\|x\|^{5}}+\frac{m_{1} m_{3} \alpha^{2}}{\|\alpha x+y\|^{3}}-\frac{3 m_{1} m_{3} \alpha^{2}\left(\alpha x^{i}+y^{i}\right)^{2}}{\|\alpha x+y\|^{5}}+ \\
& +\frac{m_{2} m_{3}}{\|\beta x-y\|^{3}}-\frac{3 m_{2} m_{3}\left(\beta x^{i}-y^{i}\right)^{2}}{\|\beta x-y\|^{5}}
\end{aligned}
$$

e

$$
\begin{aligned}
\frac{\partial^{2} V}{\partial y^{i^{2}}} & =\frac{m_{1} m_{3}}{\|\alpha x+y\|^{3}}-\frac{3 m_{1} m_{3}\left(\alpha x^{i}+y^{i}\right)^{2}}{\|\alpha x+y\|^{5}}+\frac{m_{2} m_{3}}{\|\beta x-y\|^{3}} \\
& -\frac{3 m_{2} m_{3}\left(\beta x^{i}-y^{i}\right)^{2}}{\|\beta x-y\|^{5}}
\end{aligned}
$$

e substituindo $\mathrm{g}^{\mathrm{ij}}$, implícito na expressão 4.4.f, junto com $\frac{\partial^{2} V}{\partial x^{i^{2}}}$ e $\frac{\partial^{2} V}{\partial y^{i^{2}}}$ na expressão de $\Delta \mathrm{V}$, segue a demontração.

$$
\text { c.q.d. }
$$

O próximo resultado é uma consequência direta do fato que o Laplaciano é identicamente nulo e a dimensão da variedade é 6 . 
Proposição 4.4.3. Para o problema de três partículas em $\mathrm{E}^{3}$ a primeira função curvatura e a curvatura escalar mecânica são ambas identicamente nulas.

Demonstração: para o nosso caso partícular, $\mathrm{n}=6 \mathrm{e} \Delta \mathrm{V}=0$, substituindo na equação 3.2.d, segue que

$$
e^{2 \rho} R^{*}=R=0
$$

Para mostrar que $\mathrm{Q}_{1}$ é nulo, basta olhar para a expressão obtida de $\mathrm{q}_{1}$, ou seja

$$
\begin{aligned}
& q_{1}(C)=\frac{n-2}{2}\left[e^{2 \rho} R^{*}-R\right] \\
& Q_{1}(x)=q_{1}\left(C^{*}(x)\right) \equiv 0 .
\end{aligned}
$$

Proposição 4.4.4. No caso em que o movimento é planar,

$$
\Delta V=-\frac{m_{1}+m_{2}}{\|x\|^{3}}-\frac{m_{1}+m_{3}}{\|\alpha x+y\|^{3}}-\frac{m_{2}+m_{3}}{\|\beta X-y\|^{3}}
$$

Demonstração: Considere $\mathrm{x}^{3}, \mathrm{y}^{3}$ ambos nulos, então

$$
\begin{aligned}
\frac{\partial^{2} V}{\partial x^{i^{2}}} & =\frac{m_{1} m_{2}}{\|x\|^{3}}-\frac{3 m_{1} m_{2} x^{i^{2}}}{\|x\|^{5}}+\frac{m_{1} m_{3} \alpha^{2}}{\|\alpha x+y\|^{3}}-\frac{3 m_{1} m_{3} \alpha^{2}\left(\alpha x^{i}+y^{i}\right)^{2}}{\|\alpha x+y\|^{5}}+ \\
& +\frac{m_{2} m_{3}}{\|\beta x-y\|^{3}}-\frac{3 m_{2} m_{3} \beta^{2}\left(\beta x^{i}-y^{i}\right)^{2}}{\|\beta x-y\|^{5}}
\end{aligned}
$$




$$
\begin{aligned}
\frac{\partial^{2} V}{\partial y i^{2}} & =\frac{m_{1} m_{3}}{\|\alpha x+y\|^{3}}-\frac{3 m_{1} m_{3}\left(\alpha x^{i}+y^{i}\right)^{2}}{\|\alpha x+y\|^{5}}+\frac{m_{2} m_{3}}{\|\beta x-y\|^{3}} \\
& -\frac{3 m_{2} m_{3}\left(\beta x^{i}-y^{i}\right)^{2}}{\|\beta x-y\|^{5}}
\end{aligned}
$$

para $\mathrm{i}=1,2$ e $\frac{\partial^{2} V}{\partial x^{3^{2}}}=0$

assim

$$
\frac{\partial^{2} V}{\partial x^{1^{2}}}+\frac{\partial^{2} V}{\partial x^{2^{2}}}=-\frac{m_{1} m_{2}}{\|x\|^{3}}-\frac{m_{1} m_{3} \alpha^{2}}{\|\alpha x+y\|^{3}}-\frac{m_{2} m_{3} \beta^{2}}{\|\beta x-y\|^{2}}
$$

da mesma forma

$$
\begin{aligned}
\frac{\partial^{2} V}{\partial y^{i^{2}}} & =\frac{m_{1} m_{3}}{\|\alpha x+y\|^{3}}-\frac{3 m_{1} m_{3}\left(\alpha x^{i}+y^{i}\right)^{2}}{\|\alpha x+y\|^{5}}+\frac{m_{2} m_{3}}{\|\beta x-y\|^{3}} \\
& -\frac{3 m_{2} m_{3}\left(\beta x^{i}-y^{i}\right)^{2}}{\|\beta x-y\|^{5}}
\end{aligned}
$$

para $\mathrm{i}=1,2$

$\mathrm{e} \frac{\partial^{2} V}{\partial y^{3^{2}}}=0$

assim

$$
\frac{\partial^{2} V}{\partial y^{1^{2}}}+\frac{\partial^{2} V}{\partial y^{2^{2}}}=-\frac{m_{1} m_{3}}{\|\alpha x+y\|^{3}}-\frac{m_{2} m_{3}}{\|\beta x-y\|^{3}}
$$


$\log 0$

$$
\begin{aligned}
\Delta V & =\frac{1}{g_{1}}\left(-\frac{m_{1} m_{2}}{\|x\|^{3}}-\frac{m_{1} m_{3} \alpha^{2}}{\|\alpha x+y\|^{3}}-\frac{m_{2} m_{3} \beta^{2}}{\|\beta x-y\|^{3}}\right)+ \\
& +\frac{1}{g_{2}}\left(-\frac{m_{1} m_{3}}{\|\alpha x+y\|^{3}}-\frac{m_{2} m_{3}}{\|\beta x-y\|^{3}}\right) \\
& =-\frac{m_{1}+m_{2}}{\|x\|^{2}}-\frac{m_{1}+m_{3}}{\|\alpha x+y\|^{3}}-\frac{m_{2}+m_{3}}{\|\beta x+y\|^{3}}
\end{aligned}
$$

c.q.d.

A seguir, mostraremos que, contrariando ao que está enunciado em [ 8 ], o potencial V não tem pontos críticos, o que implica que as variedades diferenciáveis h-mecânicas estão bem definidadas para qualquer valor de energia h.

Proposição 4.4.5. Para todas as massas $\left(\mathrm{m}_{1}, \mathrm{~m}_{2}, \mathrm{~m}_{3}\right) \in \mathrm{R}^{+} \times \mathrm{R}^{+} \times \mathrm{R}^{+}$, qualquer valor de energia h é um valor regular da função Potencial Newtoniana.

Demonstração: $\mathrm{O}$ valor de energia $\mathrm{h}$ deixa de ser um valor regular do potencial $\mathrm{V}$ quando $\mathrm{dV}(\mathrm{x}, \mathrm{y})=0$ para algum $(\mathrm{x}, \mathrm{y}) \in \mathrm{V}^{-1}(\mathrm{~h})$. Por outro lado,

$$
\mathrm{dV}=0 \text { se, e somente se, } \mathrm{V}_{\mathrm{x}}=\mathrm{V}_{\mathrm{y}}=0 \text {, mas }
$$

4.4.f.

$$
V_{y}=0 \leftrightarrow \frac{m_{1}(\alpha x+y)}{\|\alpha x+y\|^{3}}=\frac{m_{2}(\beta x-y)}{\|\beta x-y\|^{3}} .
$$

Calculando as normas em ambos os membros,

$$
\frac{m_{1}}{\|\alpha x+y\|^{2}}=\frac{m_{2}}{\|\beta x-y\|^{2}}
$$


Voltando à equação 4.4.f,

4.4.g. $\quad \beta x-y=\left(\frac{m_{2}}{m_{1}}\right)^{\frac{1}{2}}(\alpha x+y)$.

Por outro lado, $\mathrm{V}_{\mathrm{x}}=\mathrm{V}_{\mathrm{y}}=0$, donde

4.4.h.

$$
\begin{aligned}
\frac{m_{1} m_{2} x}{\|x\|^{3}} & =-\left(\frac{m_{1} m_{3} \alpha(\alpha x+y)}{\|\alpha x+y\|^{3}}+\frac{m_{2} m_{3} \beta(\beta x-y)}{\|\beta x-y\|^{3}}\right) \\
& =-\frac{m_{1} m_{3}(\alpha x+y)(\alpha+\beta)}{\|\alpha x+y\|^{3}}
\end{aligned}
$$

Novamente, calculando as normas em ambos os membros, e usando a definição de $\alpha, \beta$ e $\eta$,

$$
\frac{m_{2}}{\|x\|^{2}}=\frac{m_{3}}{\|\alpha X+y\|^{2}}
$$

Voltando a equação 4.4.h,
4.4.i.
$\alpha x+y=-\sqrt{\frac{m_{3}}{m_{2}}} x$.

De 4.4.g segue que:

4.4.j.

$$
y=-\left(\left(\frac{m_{3}}{m_{2}}\right)^{\frac{1}{2}}+\alpha\right) x
$$

Substituindo 4.4.j em 4.4.g,

4.4.1.

$$
y=\left(\beta+\sqrt{\frac{m_{3}}{m_{1}}}\right) x
$$


subtraindo 4.4.j de 4.4.1, lembrando que x não é nulo em $M$, segue que:

$$
\beta+\sqrt{\frac{m_{3}}{m_{1}}}+\sqrt{\frac{m_{3}}{m_{2}}}+\alpha=0
$$

completando assim a demonstração.

Supondo que ocorre uma única colisão entre as massas $\mathrm{m}_{1}$ e $\mathrm{m}_{2}$, isto significa que $\left\|x_{2}-x_{1}\right\|=\|x\| \rightarrow 0$ quando as partículas tendem à colisão. Embora

$\mathrm{x}=0$ não esteja em $\mathrm{M}$, vamos estudar o comportamento da variedade em uma vizinhança deste ponto.

Usando as expressões de $\Delta \mathrm{V}, N_{\mathrm{V}^{2}}$ e substituindo na expressão de $\mathrm{R}^{*}$, segue que:

$$
\begin{aligned}
R^{*} & =\frac{6}{e^{6 \rho}}\left[(h-V) \Delta V+N_{v^{2}}\right] \\
& =\frac{6}{e^{6 \rho}}\left[-\left(h+\frac{m_{1} m_{2}}{\|x\|}+\frac{m_{1} m_{3}}{\|\alpha x+y\|}+\frac{m_{2} m_{3}}{\|\beta x-y\|}\right)\left(\frac{m_{1}+m_{2}}{\|x\|^{3}}+\frac{m_{1}+m_{3}}{\|\alpha x+y\|^{3}}+\frac{m_{2}+m_{3}}{\|\beta x-y\|^{3}}\right)\right. \\
& +\frac{m_{1} m_{2}\left(m_{1}+m_{2}\right)}{\|x\|^{4}}+\frac{m_{1} m_{3}\left(m_{1}+m_{3}\right)}{\|\alpha x+y\|^{4}}+\frac{m_{2} m_{3}\left(m_{2}+m_{3}\right)}{\|b e t a x-y\|^{4}} \\
& +\left\langle\frac{2 m_{1} m_{2} m_{3} x}{\|x\|^{3}}, \frac{\alpha x+y}{\|\alpha x+y\|^{3}}+\frac{\beta x-y}{\|\beta x-y\|^{3}}\right\rangle-\left\langle\frac{2 m_{1} m_{2} m_{3}(\alpha x+y)}{\|\alpha x+y\|^{3}}, \frac{\beta x-y}{\|\beta x-y\|^{3}}\right\rangle \\
& =\frac{6}{e^{6} \rho}\left[-\left(h+\frac{m_{1} m_{2}}{\|x\|}+A\right)\left(\frac{m_{1}+m_{2}}{\|x\|^{3}}+B\right)+\frac{m_{1} m_{2}\left(m_{1}+m_{2}\right)}{\|x\|^{4}}+O\left(\frac{1}{\|x\|^{2}}\right)\right]
\end{aligned}
$$

onde

$$
\begin{aligned}
& A=\frac{m_{1} m_{3}}{\|\alpha x+y\|}+\frac{m_{2} m_{3}}{\|\beta x-y\|} \\
& B=\frac{m_{1}+m_{3}}{\|\alpha x+y\|^{3}}+\frac{m_{2}+m_{3}}{\|\beta x-y\|^{3}}
\end{aligned}
$$

assim 


$$
\begin{aligned}
R^{*} & =-\frac{6}{e^{6 p}}\left[h \left(\frac{m_{1}+m_{2}}{\|x\|^{3}}+h B-\frac{m_{1} m_{2}\left(m_{1}+m_{2}\right)}{\|x\|^{4}}+\frac{m_{1} m_{2}}{\|x\|} B+\frac{A\left(m_{1}+m_{2}\right)}{\|x\|^{3}}+\right.\right. \\
& +A B+\frac{m_{1} m_{2}\left(m_{1}+m_{2}\right)}{\|x\|^{4}}-O\left(\frac{1}{\|x\|^{2}}\right) \\
& =\frac{6}{e^{6 p}}\left[-\frac{\left(m_{1}+m_{2}\right)}{\|x\|^{3}}(h+A)+O\left(\frac{1}{\|x\|^{2}}\right]\right.
\end{aligned}
$$

Assim, o sinal de $\mathrm{R}^{*}$ na vizinhança de uma colisão depende apenas de

$$
\Omega=h+A
$$

Observação: Na colisão, $\mathrm{d}\left(\mathrm{m}_{3}, \mathrm{~m}_{1}\right)=\mathrm{d}\left(\mathrm{m}_{3}, \mathrm{~m}_{2}\right)=\mathrm{L}$.

Próximo de uma colisão para um alto valor da energia total h, a curvatura mecânica escalar $\mathrm{R}^{*}$ é negativa. E se h é negativo, então para as distâncias entre as partículas suficientemente grandes, $\mathrm{R}^{*}$ é positivo, como é mostrado a seguir.

Proposição 4.4.6. Para o problema de três partículas planar, em uma vizinhança da única colisão entre massas $m_{1}$ e $m_{2}$, temos que

$$
R^{*}>0 \quad(<0) \leftrightarrow h+\frac{m_{3}\left(m_{1}+m_{2}\right)}{L}<0 \quad(>0) .
$$

A demonstração segue do fato que

$$
\begin{gathered}
\|\alpha x+y\|=\left\|x_{3}-x_{1}\right\|=L \\
\|\beta x-y\|=\left\|x_{2}-x_{3}\right\|=L \\
\Omega=h+\frac{m_{3} \mu}{L}
\end{gathered}
$$

Esse teorema afirma que próximo de uma colisão, para um alto valor da energia, a curvatura mecânica escalar $\mathrm{R}^{*}$ é negativa. E se h é negativo, então para 
a distância $\mathrm{L}$ do teorema suficientemente grande, $\mathrm{R}^{*}$ é positivo.

Para o cálculo das funções curvaturas $\mathrm{Q}_{\mathrm{j}}$ definidas em 3.4.6, se tomarmos $\lambda_{\mathrm{i}}, \mathrm{i}=1,2,3,4$ como auto valores de $\mathrm{C}^{*} \mathrm{e}$

$$
\begin{aligned}
& \mu_{1}=\lambda_{1}+\lambda_{2}, \quad \mu_{2}=\lambda_{1}+\lambda_{3}, \quad \mu_{3}=\lambda_{1}+\lambda_{4}, \\
& \mu_{4}=\lambda_{2}+\lambda_{3}, \quad \mu_{5}=\lambda_{2}+\lambda_{4}, \quad \mu_{6}=\lambda_{3}+\lambda_{4} .
\end{aligned}
$$

então

$$
\begin{aligned}
q_{1}(\lambda) & =\sum_{i=1}^{6} \mu_{i}=3 \sum_{i=1}^{4} \lambda_{i} \\
q_{2}(\lambda) & =\sum_{i<j} \mu_{i} \mu_{j}=3 \sum_{i=1}^{4} \lambda_{i}^{2}+8 \sum_{i<j} \lambda_{i} \lambda_{j} \\
q_{3}(\lambda) & =\sum_{i<j<k} \mu_{i} \mu_{j} \mu_{k}=\sum_{i=1}^{4} \lambda_{i}^{3}+7 \sum_{i, j}^{4} \lambda_{i}^{2} \lambda_{j}+18 \sum_{i<j<k} \lambda_{i} \lambda_{j} \lambda_{k} \\
q_{4}(\lambda) & =\sum_{i<j<k<1} \mu_{i} \mu_{j} \mu_{k} \mu_{1} \\
& =2 \sum_{i, j}^{4} \lambda_{i}^{3} \lambda_{j}+5 \sum_{i<j} \lambda_{i}^{2} \lambda_{j}^{2}+13 \sum_{i, j<k} \lambda_{i}^{2} \lambda_{j} \lambda_{k} \\
q_{5}(\lambda) & =\sum_{i, j, k, 1, m} \mu_{i} \mu_{j} \mu_{k} \mu_{1} \mu_{m} \\
& =\sum_{i \neq j} \lambda_{i}^{3} \lambda_{j}^{2}+3 \sum_{i \neq j<k} \lambda_{i}^{3} \lambda_{j} \lambda_{k}+7 \sum_{i<j \neq k} \lambda_{i}^{2} \lambda_{j}^{2} \lambda_{k}+15 \sum_{i \neq j<k<1} \lambda_{i}^{2} \lambda_{j} \lambda_{k} \lambda_{1} \\
q_{6}(\lambda) & =\mu_{1} \mu_{2} \mu_{3} \mu_{4} \mu_{5} \mu_{6} \\
& =\sum_{i, j, k} \lambda_{i}^{3} \lambda_{j}^{2} \lambda_{k}+2 \sum_{i \neq j<k<1} \lambda_{i}^{3} \lambda_{j} \lambda_{k} \lambda_{1}+2 \sum_{i<j<k} \lambda_{i}^{2} \lambda_{j}^{2} \lambda_{k}^{2}+4 \sum_{i<j \neq k<1} \lambda_{i}^{2} \lambda_{j}^{2} \lambda_{k} \lambda_{1}
\end{aligned}
$$


Como $\Gamma_{j k}^{i}$ são nulos, a expressão de $\mathrm{C}_{\mathrm{jk}}$ fica

$$
C_{j k}=\frac{2}{e^{4 \rho}}\left[2(h-V) V_{j k}+3 V_{j} V_{k}-\frac{1}{2} N_{V^{2}} g_{j k}\right]
$$

ou seja, o operador auto-adjunto $C^{*}$ pode ser escrito em função de $V$ e suas derivadas.

Apesar das dificuldades dos cálculos dos auto-valores da matriz $\mathrm{C}_{\mathrm{jk}}$ de forma analítica e, portanto, não tendo explícita as expressões das funções $\mathrm{q}_{\mathrm{j}}$, é possível obter esses cálculos de forma pontual. Assim, podemos aplicar as proposições $3.4 .7,3.5 .1,3.5 .3,3.5 .4$ e 3.5 .5 . 


\section{\# 4.5. O Pêndullo Duplo}

Um pêndulo duplo move-se em um plano vertical. Duas coordenadas $\theta_{1}, \theta_{2}$ definem as posições das massas $\mathrm{m}_{1}$ e $\mathrm{m}_{2}$, conforme figura abaixo.

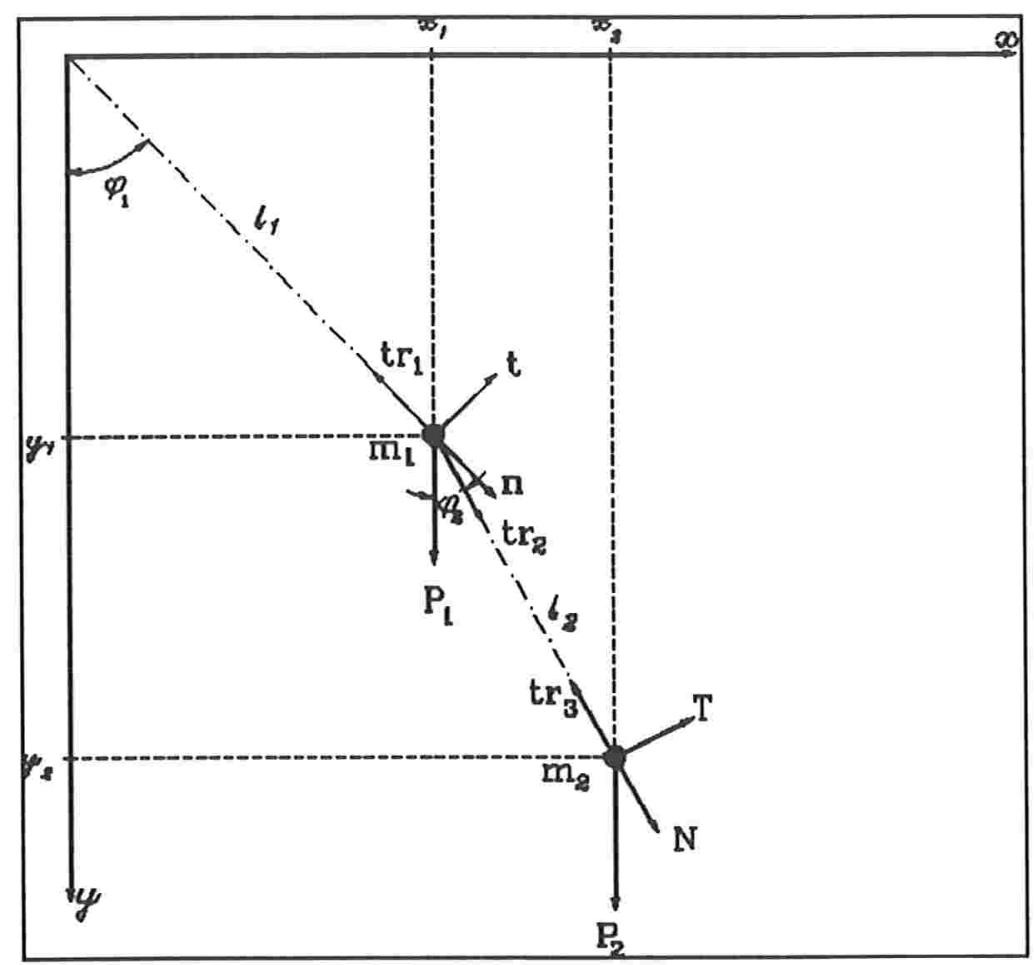

figura 4.5.1

Em coordenadas cartesianas tem-se que:

$$
\begin{aligned}
& x_{1}=I_{1} \operatorname{sen} \theta_{1} \\
& y_{1}=I_{1} \cos \theta_{1} \\
& x_{2}=I_{1} \operatorname{sen} \theta_{1}+I_{2} \operatorname{sen} \theta_{2} \\
& y_{2}=I_{1} \cos \theta_{1}+I_{2} \cos \theta_{2}
\end{aligned}
$$

O espaço de configuração é dado por:

$\mathbf{M}=\left\{\left(\theta_{1}, \theta_{2}\right) \in[-\pi, \pi] \times[-\pi, \pi]\right\}$ ou seja, identificando os pontos do bordo do retângulo, é o toro parametrizado pelas coordenadas $\theta_{1}, \theta_{2}$. E a energia cinética é, 


$$
K=\frac{m_{1}}{2}\left\langle\left(\dot{x}_{1}, \dot{y}_{1}\right),\left(\dot{x}_{1}, \dot{y}_{1}\right)\right\rangle+\frac{m_{2}}{2}\left\langle\left(\dot{x}_{2}, \dot{y}_{2}\right),\left(\dot{x}_{2}, \dot{y}_{2}\right)\right\rangle
$$

ou nas coordenadas $\theta_{1}, \theta_{2}$

4.5.a. $K\left(\dot{\theta}_{1}, \dot{\theta}_{2}\right)=\frac{m_{1}}{2} I_{1}^{2} \dot{\theta}_{1}^{2}+\frac{m_{2}}{2}\left[I_{1}^{2} \dot{\theta}_{1}^{2}+I_{2}^{2} \dot{\theta}_{2}^{2}+2 I_{1} I_{2} \theta_{1} \dot{\theta}_{2} \cos \left(\theta_{2}-\theta_{1}\right)\right]$

A energia potencial $\mathrm{V}$ é dada por:

$$
\mathrm{V}=-\mathrm{P}_{1} \mathrm{~h}_{1}-\mathrm{P}_{2} \mathrm{~h}_{2}
$$

onde $\mathrm{P}_{\mathrm{i}}$ e $\mathrm{h}_{\mathrm{i}}$ são as forças peso e as alturas correspondentes as massas $\mathrm{m}_{\mathrm{i}}, \mathrm{i}=1,2$.

Ou ainda, em coordenadas polares:

4.5.b.

$$
V\left(\theta_{1}, \theta_{2}\right)=-g I_{1}\left(m_{1}+m_{2}\right) \cos \theta_{1}-m_{2} g I_{2} \cos \theta_{2}
$$

Com estes entes mecânicos e geométricos, fica descrito o sistema mecânico simples $(\mathrm{M}, \mathrm{K}, \mathrm{V})$.

A seguir, é dada a forma explícita da métrica Riemanniana g. Para isto, considere $\mathrm{x}$ uma carta local em $\mathrm{p} \in \mathrm{M}$ e escrevendo:

$$
\begin{aligned}
& X=X_{1} \frac{\partial}{\partial \theta_{1}}+X_{2} \frac{\partial}{\partial \theta_{2}} \\
& Y=Y_{1} \frac{\partial}{\partial \theta_{1}}+Y_{2} \frac{\partial}{\partial \theta_{2}}
\end{aligned}
$$

usando as relações,

4.5.c.

$$
K\left(\dot{\theta}_{1}, \dot{\theta}_{2}\right)=\frac{1}{2} g\left(\left(\dot{\theta}_{1}, \dot{\theta}_{2}\right),\left(\dot{\theta}_{1}, \dot{\theta}_{2}\right)\right)
$$


4.5.d.

$$
g(X-Y, X-Y)=g(X, X)-2 g(X, Y)+g(Y, Y)
$$

e substituindo 4.5.a e 4.5.c em 4.5.d,

$$
g(X, Y)=m_{1} I_{1}^{2} X_{1} Y_{1}+m_{2}\left[I_{1}^{2} X_{1} Y_{1}+I_{2}^{2} X_{2} Y_{2}+I_{1} I_{2}\left(X_{1} Y_{2}+Y_{1} X_{2}\right) \cos \left(\theta_{2}-\theta_{1}\right)\right]
$$

logo, a matriz g é dada por:

$$
g=\left[\begin{array}{cc}
\left(m_{1}+m_{2}\right) I_{1}^{2} & m_{2} I_{1} I_{2} \cos \left(\theta_{2}-\theta_{1}\right) \\
m_{2} I_{1} I_{2} \cos \left(\theta_{2}-\theta_{1}\right) & m_{2} I_{2}^{2}
\end{array}\right]
$$

e através de um simples cálculo segue que:

$$
g^{-1}=\left[\begin{array}{cc}
\frac{1}{I_{1}^{2}\left[m_{1}+m_{2} \operatorname{sen}^{2}\left(\theta_{2}-\theta_{1}\right)\right]} & \frac{-\cos \left(\theta_{2}-\theta_{1}\right)}{I_{1} I_{2}\left[m_{1}+m_{2} \operatorname{sen}^{2}\left(\theta_{2}-\theta_{1}\right)\right]} \\
\frac{-\cos \left(\theta_{2}-\theta_{1}\right)}{I_{1} I_{2}\left[m_{1}+m_{2} \operatorname{sen}^{2}\left(\theta_{2}-\theta_{1}\right)\right]} & \frac{m_{1}+m_{2}}{m_{2} I_{2}^{2}\left[m_{1}+m_{2} \operatorname{sen}^{2}\left(\theta_{2}-\theta_{1}\right)\right]}
\end{array}\right]
$$

Com isto, os símbolos de Christoffel da conexão Riemanniana são dados por:

$$
\begin{aligned}
& \Gamma_{11}^{1}=\frac{-m_{2} \operatorname{sen}\left(\theta_{2}-\theta_{1}\right) \cos \left(\theta_{2}-\theta_{1}\right)}{m_{1}+m_{2} \operatorname{sen}^{2}\left(\theta_{2}-\theta_{1}\right)} \\
& \Gamma_{22}^{2}=\frac{m_{2} \operatorname{sen}\left(\theta_{2}-\theta_{1}\right) \cos \left(\theta_{2}-\theta_{1}\right)}{m_{1}+m_{2} \operatorname{sen}^{2}\left(\theta_{2}-\theta_{1}\right)} \\
& \Gamma_{22}^{1}=\frac{-m_{2} I_{2} \operatorname{sen}\left(\theta_{2}-\theta_{1}\right)}{I_{1}\left[m_{1}+m_{2} \operatorname{sen}\left(\theta_{2}-\theta_{1}\right)\right]} \\
& \Gamma_{11}^{2}=\frac{I_{1}\left(m_{1}+m_{2}\right) \operatorname{sen}\left(\theta_{2}-\theta_{1}\right)}{I_{2}\left[m_{1}+m_{2} \operatorname{sen}\left(\theta_{2}-\theta_{1}\right)\right]} \\
& \Gamma_{12}^{1}=\Gamma_{12}^{2}=0
\end{aligned}
$$


Para o cálculo da norma e o laplaciano da energia potencial $V$, são necessárias as seguintes derivadas:

$$
\begin{aligned}
& \frac{\partial V}{\partial \theta_{1}}=g I_{1}\left(m_{1}+m_{2}\right) \operatorname{sen} \theta_{1} \\
& \frac{\partial V}{\partial \theta_{2}}=m_{2} g I_{2} \operatorname{sen} \theta_{2}
\end{aligned}
$$

4.5.e.

$$
\begin{aligned}
& \frac{\partial^{2} V}{\partial \theta_{1}^{2}}=g I_{1}\left(m_{1}+m_{2}\right) \cos \theta_{1} \\
& \frac{\partial^{2} V}{\partial \theta_{2}^{2}}=m_{2} g I_{2} \cos \theta_{2}
\end{aligned}
$$

portanto,

$$
\begin{aligned}
N_{V^{2}}= & \frac{g^{2}\left(m_{1}+m_{2}\right)^{2} \operatorname{sen}^{2} \theta_{1}}{m_{1}+m_{2} \operatorname{sen}^{2}\left(\theta_{2}-\theta_{1}\right)}+\frac{m_{2} g^{2}\left(m_{1}+m_{2}\right) \operatorname{sen}^{2} \theta_{2}}{m_{1}+m_{2} \operatorname{sen}^{2}\left(\theta_{2}-\theta_{1}\right)}+ \\
& -\frac{2 g^{2} m_{2}\left(m_{1}+m_{2}\right) \cos \left(\theta_{2}-\theta_{1}\right) \operatorname{sen} \theta_{1} \operatorname{sen} \theta_{2}}{m_{1}+m_{2} \operatorname{sen}^{2}\left(\theta_{2}-\theta_{1}\right)}
\end{aligned}
$$

$\mathrm{e}$

$$
\begin{aligned}
\Delta V= & \frac{\partial^{2} V}{\partial \theta_{1}^{2}} g^{11}+\frac{\partial^{2} V}{\partial \theta_{2}^{2}} g^{22}-\sum_{i=1}^{2} \frac{\partial V}{\partial \theta_{i}}\left(\Gamma_{11}^{i} g^{11}+\Gamma_{22}^{i} g^{22}\right) \\
= & \frac{g\left(m_{1}+m_{2}\right) \cos \theta_{1}}{I_{1}\left[m_{1}+m_{2} \operatorname{sen}^{2}\left(\theta_{2}-\theta_{1}\right)\right]}+\frac{g\left(m_{1}+m_{2}\right) \cos \theta_{2}}{I_{2}\left[m_{1}+m_{2} \operatorname{sen}^{2}\left(\theta_{2}-\theta_{1}\right)\right]}+ \\
& \frac{g m_{2}\left(m_{1}+m_{2}\right) \operatorname{sen} \theta_{1} \operatorname{sen}\left(\theta_{2}-\theta_{1}\right) \cos \left(\theta_{2}-\theta_{1}\right)}{l_{1}\left[m_{1}+m_{2} \operatorname{sen}^{2}\left(\theta_{2}-\theta_{1}\right)\right]^{2}}+\frac{g\left(m_{1}+m_{2}\right)^{2} \operatorname{sen} \theta_{1} \operatorname{sen}\left(\theta_{2}-\theta_{1}\right)}{I_{2}\left[m_{1}+m_{2} \operatorname{sen}^{2}\left(\theta_{2}-\theta_{1}\right)\right]^{2}} \\
& -\frac{g m_{2}\left(m_{1}+m_{2}\right) \cos \theta_{2} \operatorname{sen}\left(\theta_{2}-\theta_{1}\right)}{I_{1}\left[m_{1}+m_{2} \operatorname{sen}^{2}\left(\theta_{2}-\theta_{1}\right)\right]^{2}}-\frac{g m_{2}\left(m_{1}+m_{2}\right) \operatorname{sen} \theta_{2} \operatorname{sen}\left(\theta_{2}-\theta_{1}\right) \cos \left(\theta_{2}-\theta_{1}\right)}{I_{2}\left[m_{1}+m_{2} \operatorname{sen}^{2}\left(\theta_{2}-\theta_{1}\right)\right]^{2}}
\end{aligned}
$$


Proposição 4.5.1. Se $\mathrm{h}$ é diferente de $\mathrm{V}(\mathrm{k} \pi, \mathrm{z} \pi), \mathrm{k}, \mathrm{z} \in \mathrm{Z}$ então $\mathrm{h}$ é um valor regular da energia potencial $\mathrm{V}$.

Demonstração: É evidente, de 4.5.e.

$$
\text { c.q.d. }
$$

Devido à proposição 4.5.1, fica claro que existem 4 valores críticos de $h$ dados por:

$$
\begin{aligned}
& \mathrm{h}_{1}=-\mathrm{g}\left(\mathrm{l}_{1}\left(\mathrm{~m}_{1}+\mathrm{m}_{2}\right)+\mathrm{m}_{2} \mathrm{l}_{2}\right), \\
& \mathrm{h}_{2}=-\mathrm{g}\left(\mathrm{l}_{1}\left(\mathrm{~m}_{1}+\mathrm{m}_{2}\right)-\mathrm{m}_{2} \mathrm{l}_{2}\right), \\
& \mathrm{h}_{3}=-\mathrm{g}\left(-\mathrm{l}_{1}\left(\mathrm{~m}_{1}+\mathrm{m}_{2}\right)+\mathrm{m}_{2} \mathrm{l}_{2}\right), \\
& \mathrm{h}_{4}=\mathrm{g}\left(\mathrm{l}_{1}\left(\mathrm{~m}_{1}+\mathrm{m}_{2}\right)+\mathrm{m}_{2} \mathrm{l}_{2}\right),
\end{aligned}
$$

A figura 4.5.2 dá uma idéia do comportamento da energia potencial e fica claro o seu ponto de mínimo. Para uma compreensão melhor, segue abaixo a figura 4.5.3, que representa as curvas de nível da função potencial. Note seus quatro valores críticos 


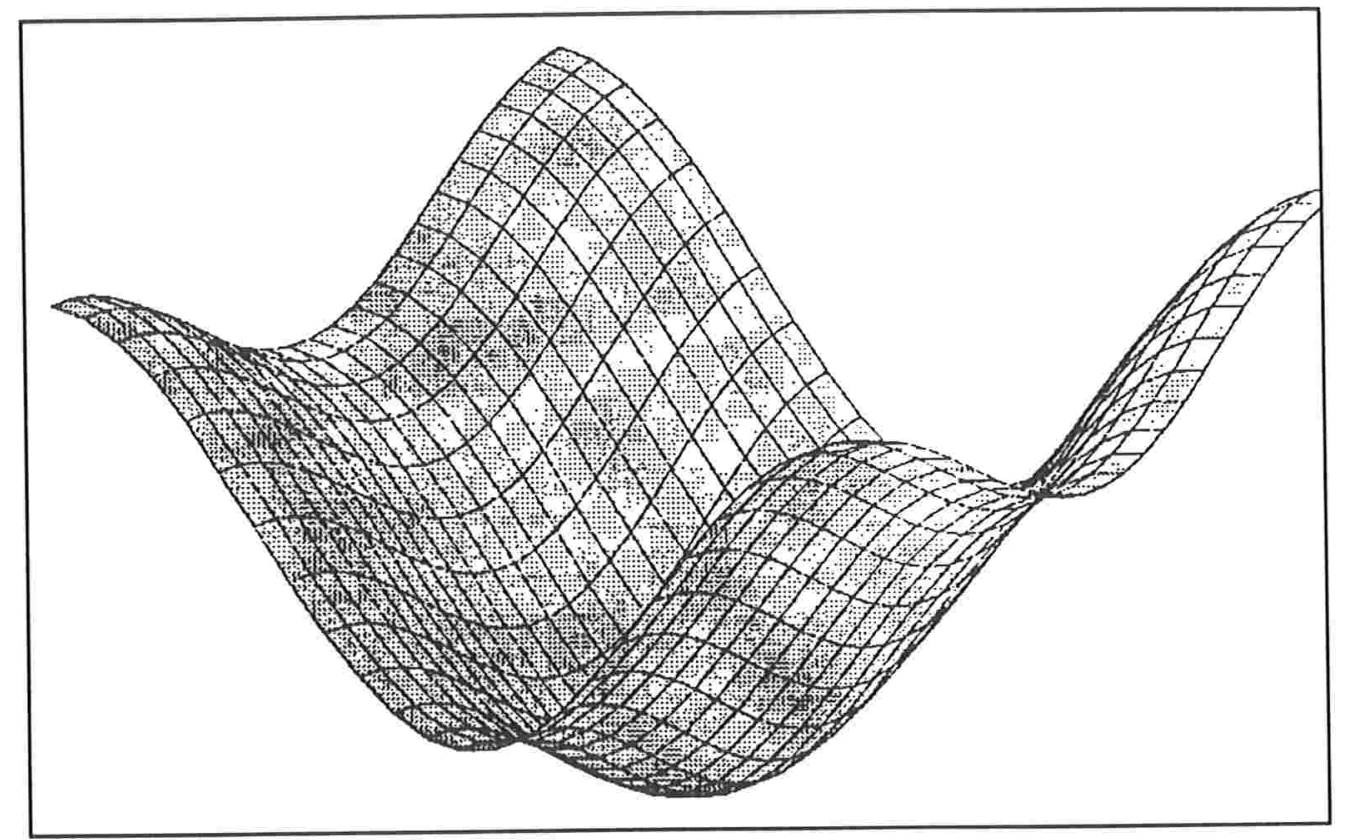

figura 4.5 .2

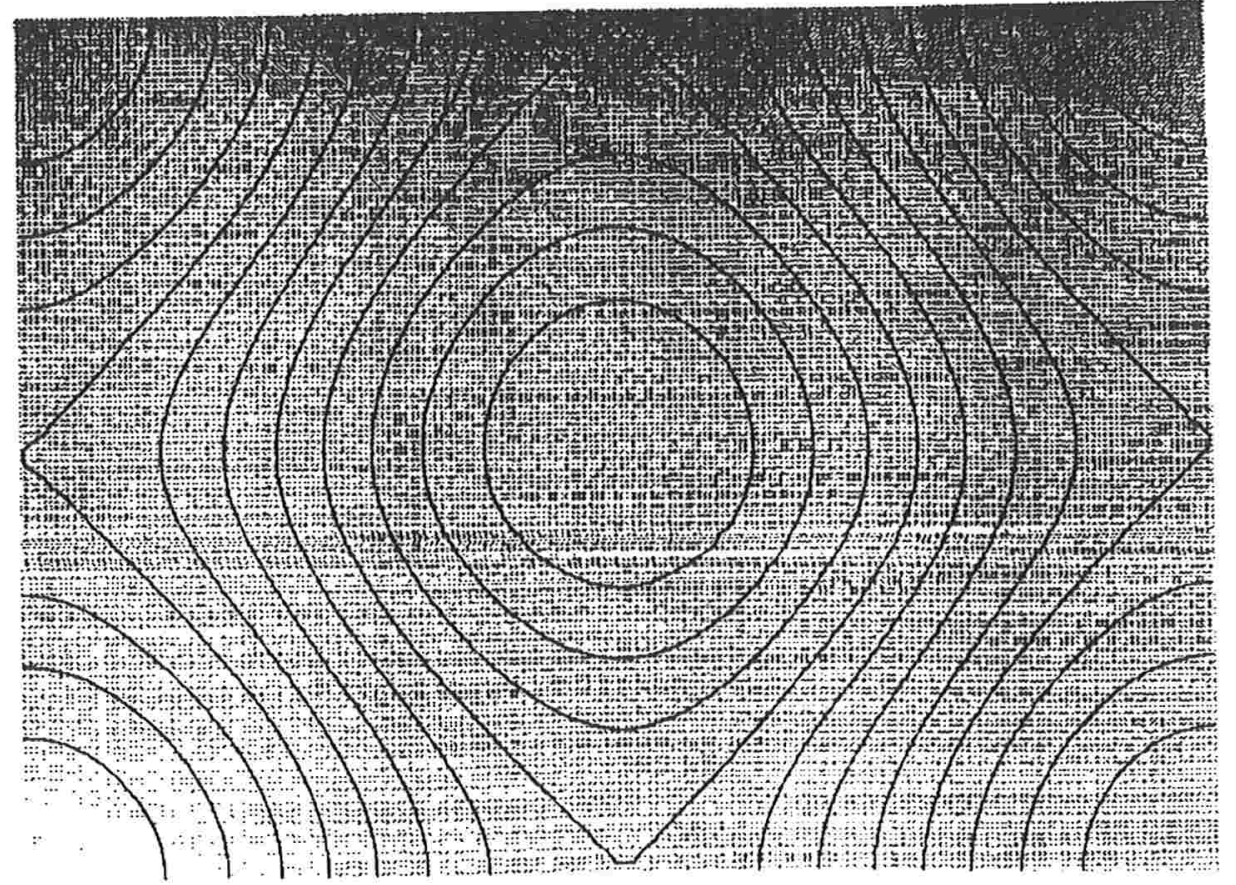

figura 4.5 .3 
A variedade Riemanniana $M_{h}$, é determinado pelo potencial V. Nas figuras 4.5.2 e 4.5.3 fica clara esta situação. Para caracterizar o bordo da variedade $M_{h}$, devemos estudar para cada $h$ as curvas que são solução da equação:

4.5.f. $\left.\quad-\mathrm{gl}_{1(} \mathrm{m}_{1}+\mathrm{m}_{2}\right) \cos \theta_{1}-\mathrm{m}_{2} \mathrm{gl}_{2} \cos \theta_{2}=\mathrm{h}$.

Para isto, as situações serão divididas em cinco casos:

CASO 1. $m_{1}$ não pode dar voltas completas.

Usando a relação 4.5.f e fazendo $\theta_{2}=0$, segue que:

4.5.g. $\quad-\mathrm{gl}_{1}\left(\mathrm{~m}_{1}+\mathrm{m}_{2}\right)-\mathrm{m}_{2} \mathrm{gl}_{2}<\mathrm{h}<\mathrm{gl}_{1}\left(\mathrm{~m}_{1}+\mathrm{m}_{2}\right)-\mathrm{m}_{2} \mathrm{gl} \mathrm{l}_{2}$

CASO 2. $m_{2}$ não pode dar voltas completas.

Mais uma vez, usando 4.5.g e fazendo $\theta_{1}=0$, segue que:

4.5.h.

$$
-\mathrm{gl}_{1}\left(\mathrm{~m}_{1}+\mathrm{m}_{2}\right)-\mathrm{m}_{2} \mathrm{gl}_{2}<\mathrm{h}<-\mathrm{gl}_{1}\left(\mathrm{~m}_{1}+\mathrm{m}_{2}\right)+\mathrm{m}_{2} \mathrm{gl} 2
$$

CASO 3. só $m_{1}$ pode ter situações de voltas completas.

Usando as relações 4.5.g e 4.5.h segue que

4.5.i. $\mathrm{gl}_{1}\left(\mathrm{~m}_{1}+\mathrm{m}_{2}\right)-\mathrm{m}_{2} \mathrm{gl}_{2}<\mathrm{h}<-\mathrm{gl}_{1}\left(\mathrm{~m}_{1}+\mathrm{m}_{2}\right)+\mathrm{m}_{2} \mathrm{gl}_{2}$

CASO 4. só $m_{2}$ pode ter situações de voltas completas.

Novamente pelas relações 4.5.g e 4.5.h, segue que:

4.5.j. $-\mathrm{gl}_{1}\left(\mathrm{~m}_{1}+\mathrm{m}_{2}\right)+\mathrm{m}_{2} \mathrm{gl}_{2}<\mathrm{h}<\mathrm{gl}_{1}\left(\mathrm{~m}_{1}+\mathrm{m}_{2}\right)-\mathrm{m}_{2} \mathrm{gl}_{2}$

CASO 5. $m_{1}$ e $m_{2}$ poderão se encontrar com situações de voltas completas.

Usando os quatro casos acima e escrevendo: 


$$
\mathrm{H}=\max \left\{\mathrm{gl}_{1}\left(\mathrm{~m}_{1}+\mathrm{m}_{2}\right)-\mathrm{m}_{2} \mathrm{gl}_{2},-\mathrm{gl}_{1}\left(\mathrm{~m}_{1}+\mathrm{m}_{2}\right)+\mathrm{m}_{2} \mathrm{gl}_{2}\right\}
$$

segue que:

4.5.1. $\quad \mathrm{H}<\mathrm{h}<\mathrm{gl}_{1}\left(\mathrm{~m}_{1}+\mathrm{m}_{2}\right)+\mathrm{m}_{2} \mathrm{gl}_{2}$.

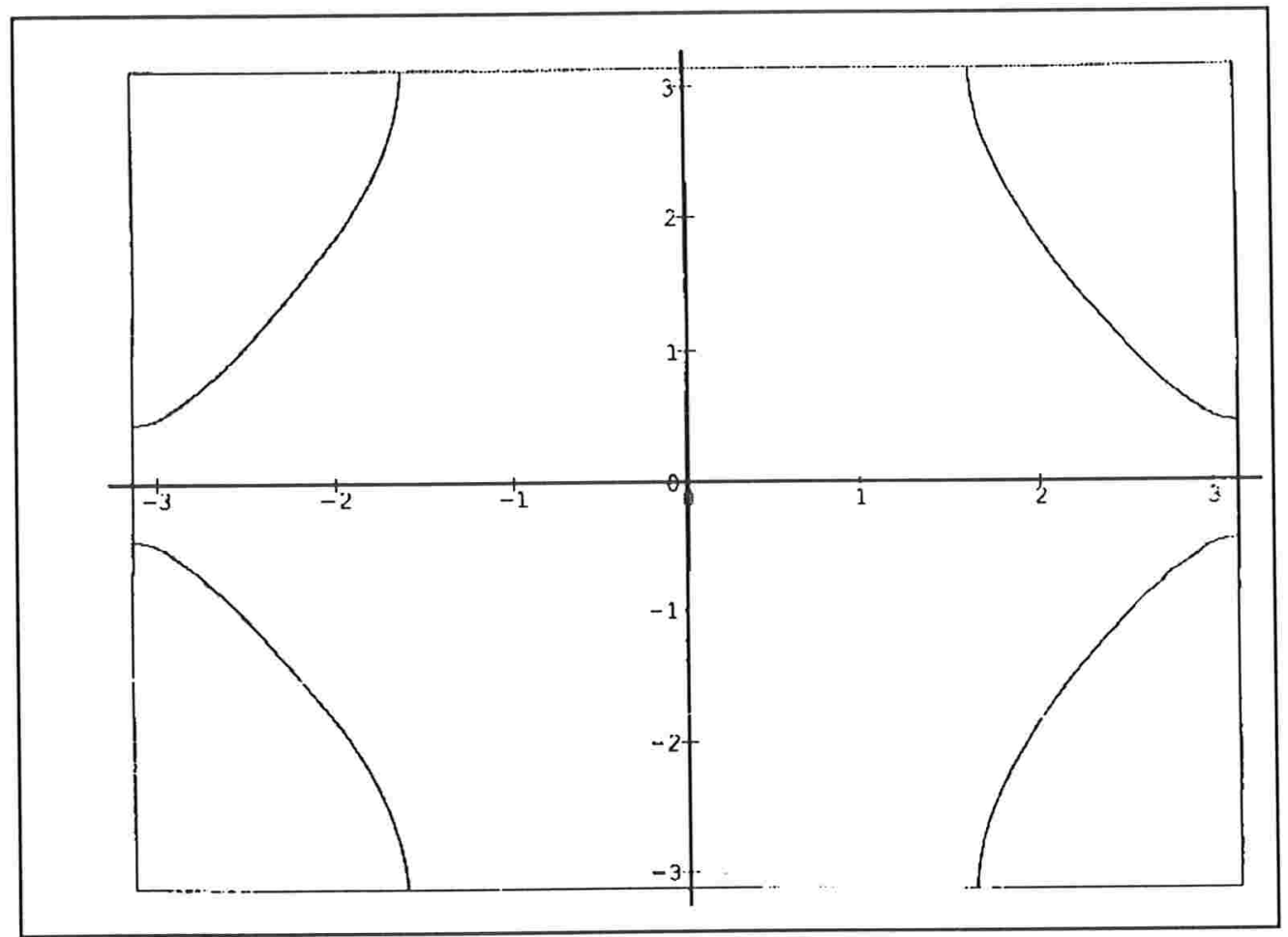

figura 4.5 .4 


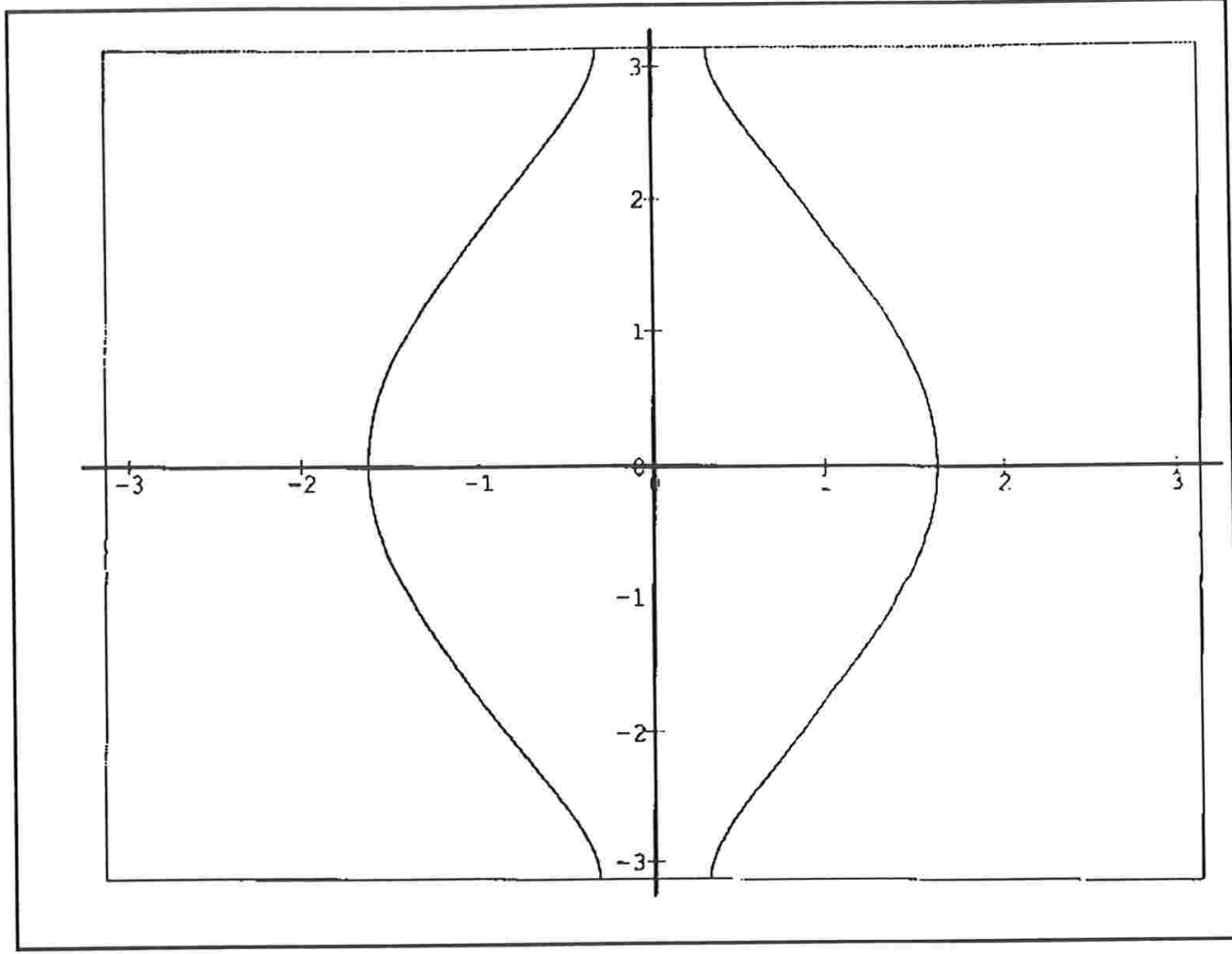

figura 4.5 .5 


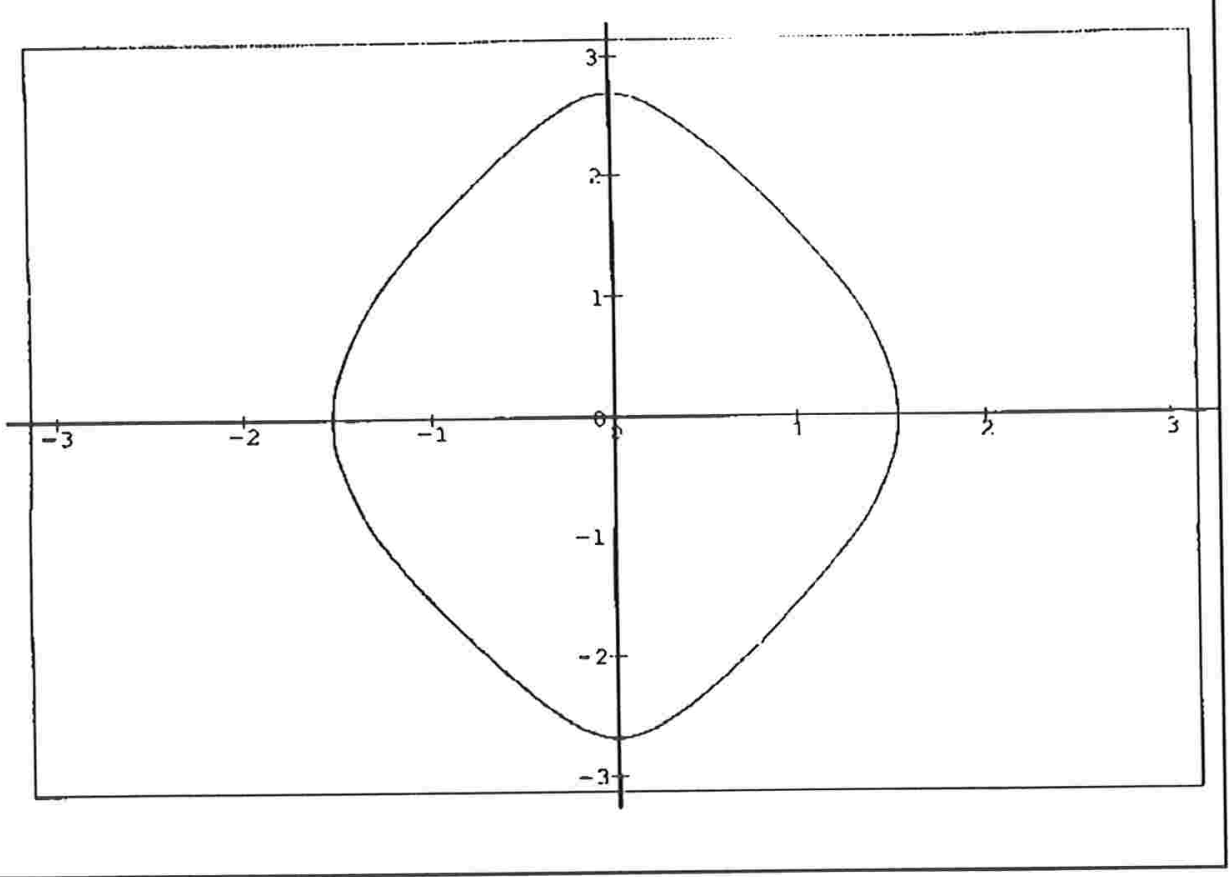

figura 4.5.6

Assim, fica caracterizado o bordo de $M_{h}$ e, portanto, $M_{h}$. Vejam por exemplo na figura 4.5 .4 a situação do caso 5 . Um exemplo dos casos 1 e 4 se encontra na figura 4.5.5 e a situação em que ambas as massas não podem dar voltas completas, é ilustrada na figura 4.5.6.

As geodésicas que fazem uma representação do movimento das massas do pêndulo, são traçadas sobre o toro. Vejam nas figuras 4.5 .7 e 4.5 .8 a situação em que a energia $\mathrm{h}$ é dada de tal forma que apenas a massa $\mathrm{m}_{2}$ pode ter situações de voltas completas. 


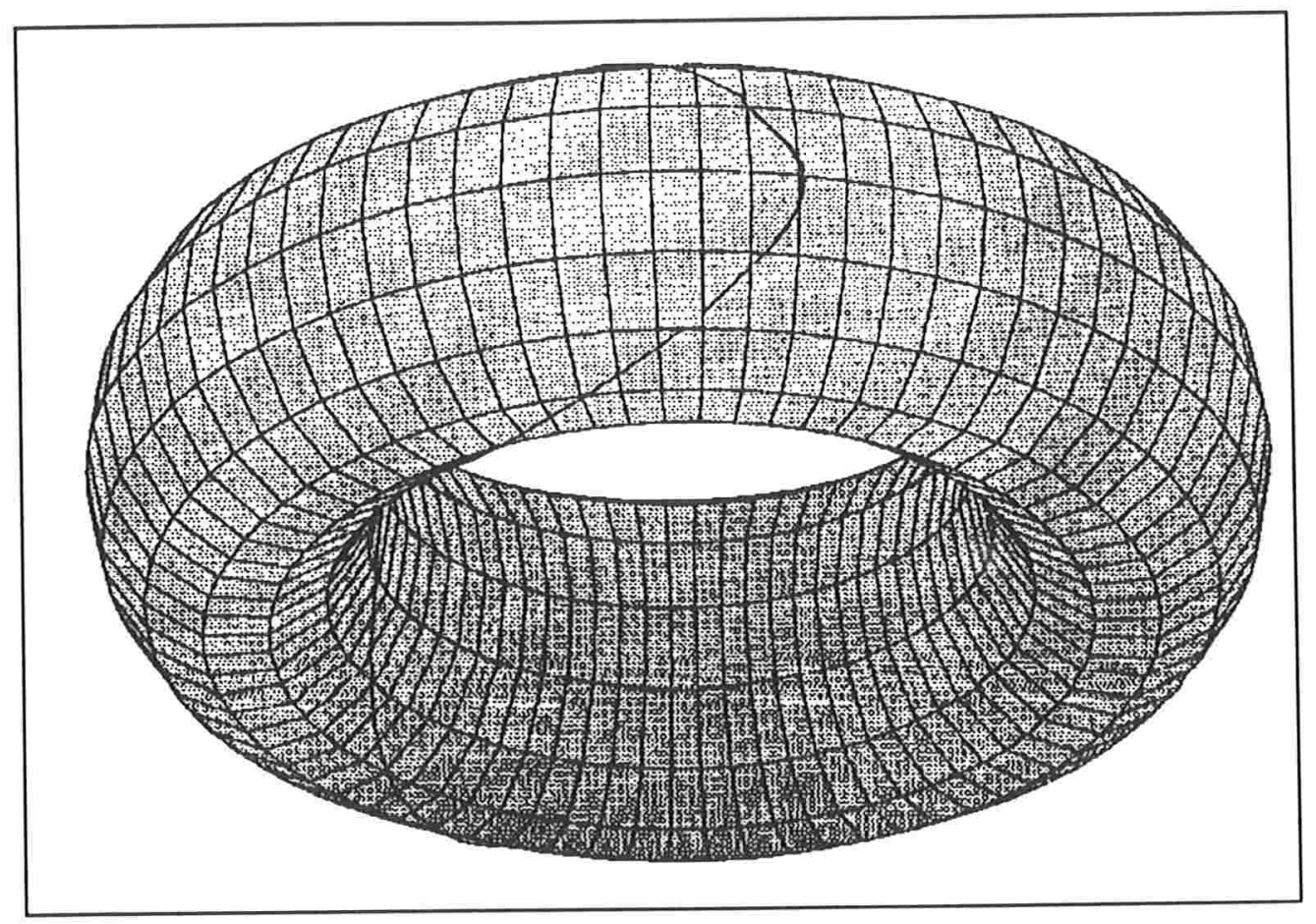

figura 4.5 .7 


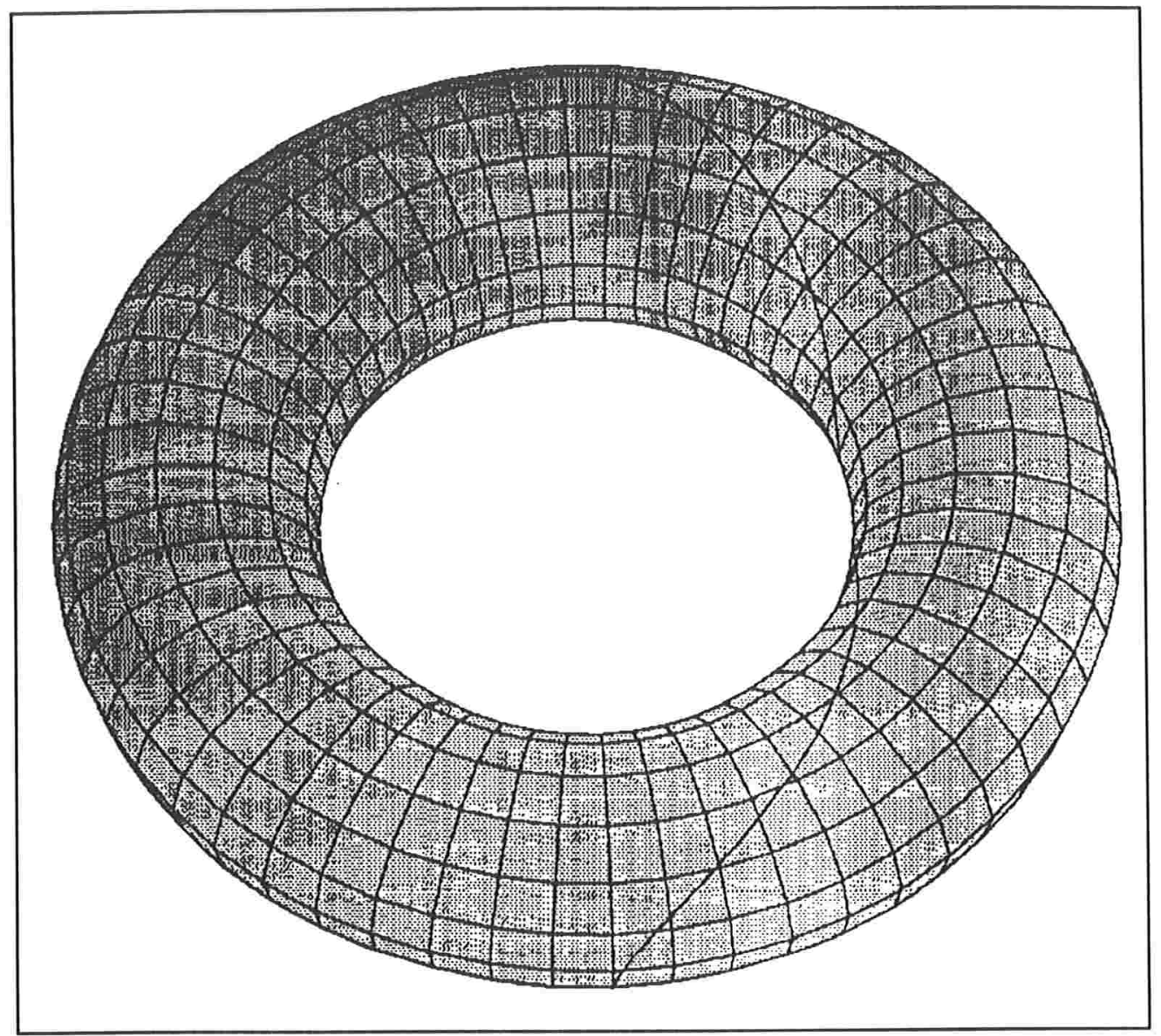

figura 4.5 .8

Usando a definição da curvatura seccional,

$$
e^{2 \rho} K^{*}(\pi)-k(\pi)=\frac{g\left(\sum_{i=1}^{2} S_{122}^{i} \frac{\partial}{\partial \theta_{i}}, \frac{\partial}{\partial \theta_{1}}\right)}{\left\|\frac{\partial}{\partial \theta_{1}} \Lambda \frac{\partial}{\partial \theta_{2}}\right\|^{2}}=0
$$

ou

$$
k^{*}(\pi)=e^{-2 p} k(\pi)
$$

logo, por cálculos diretos, obtemos a demonstração da seguinte proposição: 
Proposição 4.5.2. A curvatura Gaussiana da variedade h-mecânica $M_{h}$ é dada por:

4.5.m. $\quad K^{*}(\pi)=\frac{m_{1} \cos \left(\theta_{2}-\theta_{1}\right)\left[\left(m_{1}+m_{2}\right)-m_{2} \cos ^{2}\left(\theta_{2}-\theta_{1}\right)\right]}{2(h-V) I_{1} I_{2}\left[m_{1}+m_{2} \operatorname{sen}^{2}\left(\theta_{2}-\theta_{1}\right)\right]^{2}}$

$\mathrm{O}$ bordo $\mathrm{M}_{\mathrm{h}}$ é um conjunto de singularidades da curvatura seccional.

A variedade $M_{h}$ é formada por regiões de curvatura positiva e negativa, separadas por linhas de curvatura nula, como mostra a seguinte proposição.

Proposição 4.5.3. No problema do pêndulo duplo planar, são verdadeiras as seguintes afirmações:
a) $\quad\left|\theta_{1}-\theta_{2}\right|<\frac{\pi}{2} \rightarrow k^{*}>0$
b) $\quad \frac{\pi}{2}<\left|\theta_{1}-\theta_{2}\right|<\frac{3 \pi}{2} \rightarrow k^{*}<0$
c) $\quad \frac{3 \pi}{2}<\left|\theta_{1}-\theta_{2}\right| \rightarrow k^{*}>0$.

A demonstração é uma consequência direta de 4.5.1.

As geodésicas de $\mathrm{M}_{\mathrm{h}}$ são as trajetórias físicas do pêndulo duplo. Para o cálculo destas curvas, usamos a definição 2.1.8 para obter as equações 2.3.a, conhecidas como as equações das geodésicas. Uma outra maneira de se obter as equações do movimento é partir de 4.5.1, e trabalhar com a decomposição de forças, ou ainda usar a formulação Lagrangeana. 
As equações das geodésicas são:

$4.5 \mathrm{n}$.

$$
\begin{aligned}
& I_{1} \theta_{1} \cos \left(\theta_{1}-\theta_{2}\right)-I_{1} \theta_{1}^{2} \operatorname{sen}\left(\theta_{1}-\theta_{2}\right)+I_{2} \theta_{2}+g \operatorname{sen} \theta_{2}=0 \\
& \left(m_{1}+m_{2}\right) g \operatorname{sen} \theta_{1}+\left(m_{1}+m_{2}\right) I_{1} \theta_{1}+m_{2} I_{2} \theta_{2} \cos \left(\theta_{1}-\theta_{2}\right)+
\end{aligned}
$$

4.5.o.

$$
+m_{2} I_{2} \dot{\theta}_{2}^{2} \operatorname{sen}\left(\theta_{1}-\theta_{2}\right)=0
$$

As equações 4.5.n e 4.5.o também são chamadas de equações do movimento.

Quais são as condições para que possam existir soluções que satisfazem $\theta_{1}(\mathrm{t})$ $=\theta_{2}(\mathrm{t})$ para todo $\mathrm{t}$ ? Para que isto aconteça, é necessário que:

$$
\left[\left(m_{1}+m_{2}\right) I_{1}+m_{2} I_{2}\right] \ddot{\theta}=-\left(m_{1}+m_{2}\right) \operatorname{gsen} \theta
$$

$\mathrm{e}$

$$
\left(I_{1}+I_{2}\right) \ddot{\theta}=-g \operatorname{sen} \theta
$$

e portanto $m_{2}=m_{1}+m_{2}$, o que responde a pergunta, isto é, não existem soluções que satisfaçam $\theta_{1}(\mathrm{t})=\theta_{2}(\mathrm{t})$ para todo $\mathrm{t}$.

No apêndice deste trabalho está um programa que nós fizemos em linguagem C que utiliza o método de Runge-Kutta para achar numéricamente as soluções das equações das geodésicas. Os dados são aproveitados pelo pacote algébrico MapleV, esboçando gráficos que ajudam a exemplificar a teoria deste trabalho.

A seguir, estão 17 gráficos que representam o comportamento das geodésicas da variedade Riemanniana $M_{h}$ para variados valores de energia $h$. Os gráficos a1, 
a2, b1 e b2, correspondem ao valor de energia $h=9.5$. Só a massa $m_{2}$ pode ter situações de voltas completas, como mostram os gráficos. Houve uma pequena pertubação nas condições iniciais das geodésicas de a1 originando a geodésica b1. A pertubação foi suficiente para que a massa $m_{2}$ desse a primeira volta em sentido contrário de a1. Os gráficos pontilhados, reforçam a teoria que próximo do bordo da variedade $\mathrm{M}_{\mathrm{h}}$, a energia cinética é baixa, como é visto quando os pontos são plotados muito próximos uns aos outros.

O gráfico d1 representa um exemplo em que ambas as massas tivessem situações de voltas completas. A massa $\mathrm{m}_{2}$ encontra-se com muito mais voltas e isto está relacionado com as constantes envolvidas, bem como as condições iniciais.

Os gráficos e1, f1 e g1 dão uma idéia intuitiva de que são pertubações de uma órbita periódica estável. Esse problema fica em aberto.

A seguir, o gráfico h1 dá um exemplo de uma geodésica que apenas a massa $\mathrm{m}_{1}$ tem situações de voltas completas e il representa uma situação em que ambas as massas não podem ter situações de voltas completas.

O gráfico m1 é uma perdubação do pêndulo simples. Fica em aberto mostrar que 11 e m1 são órbitas periódicas do pêndulo duplo. 


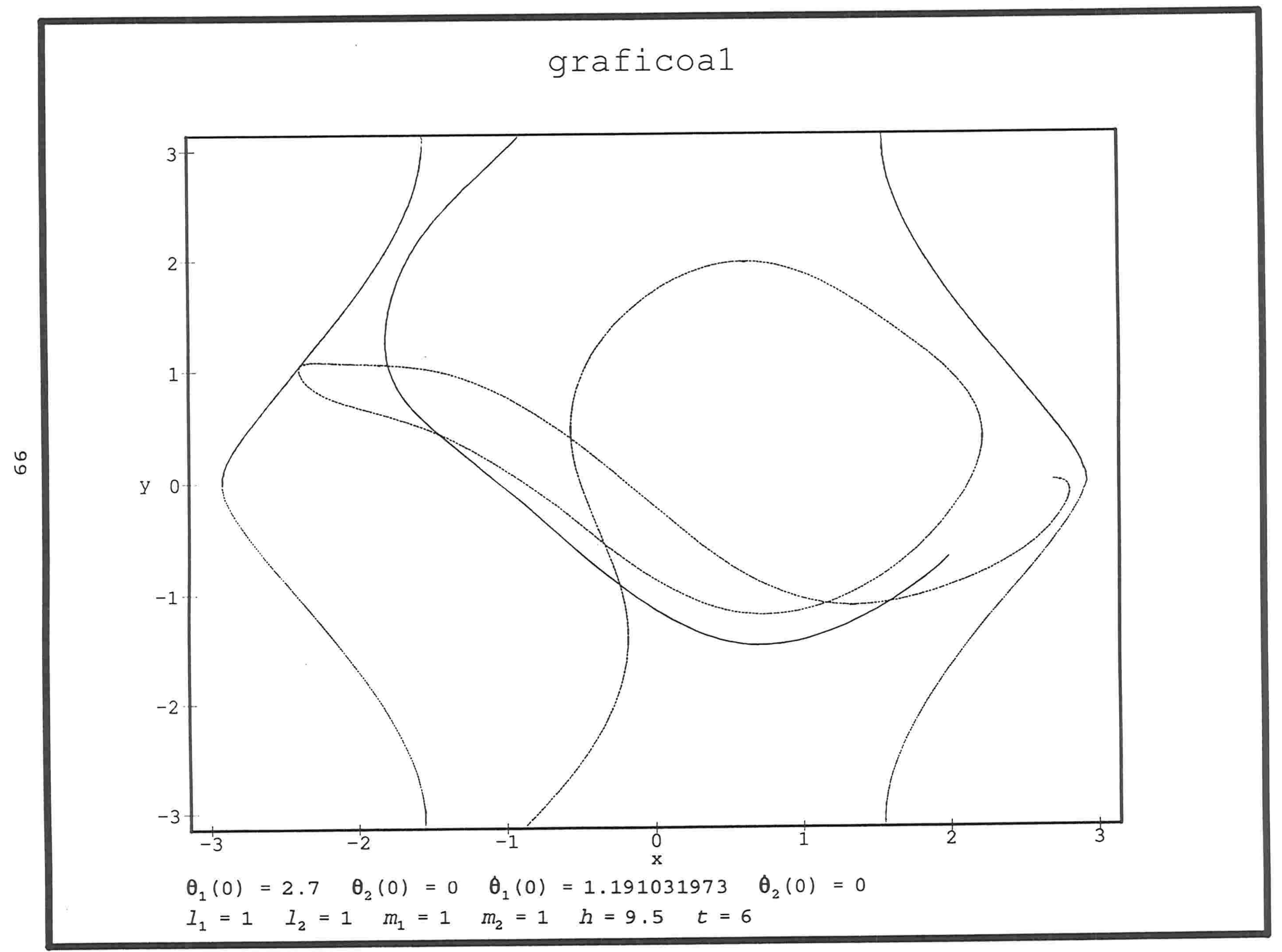




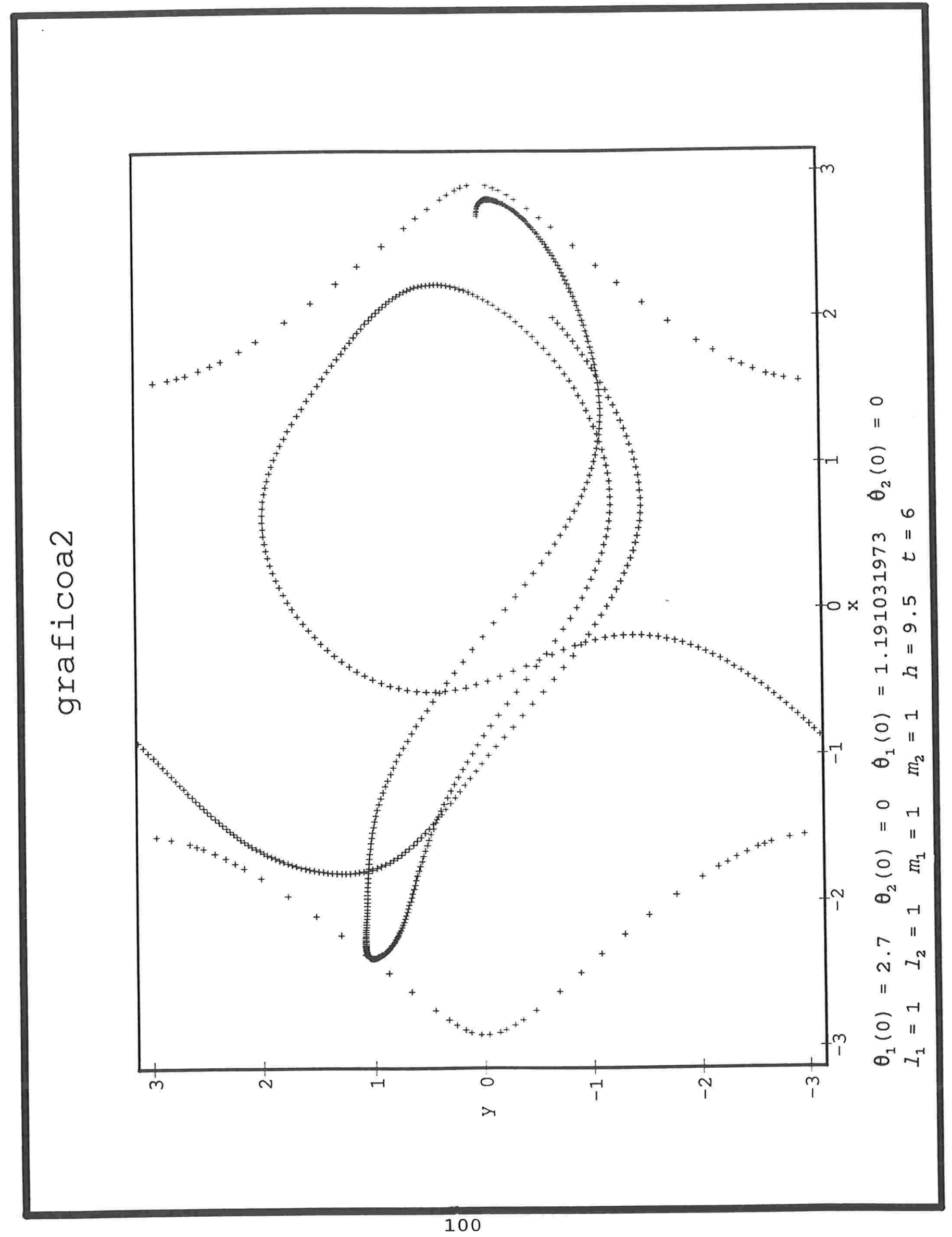




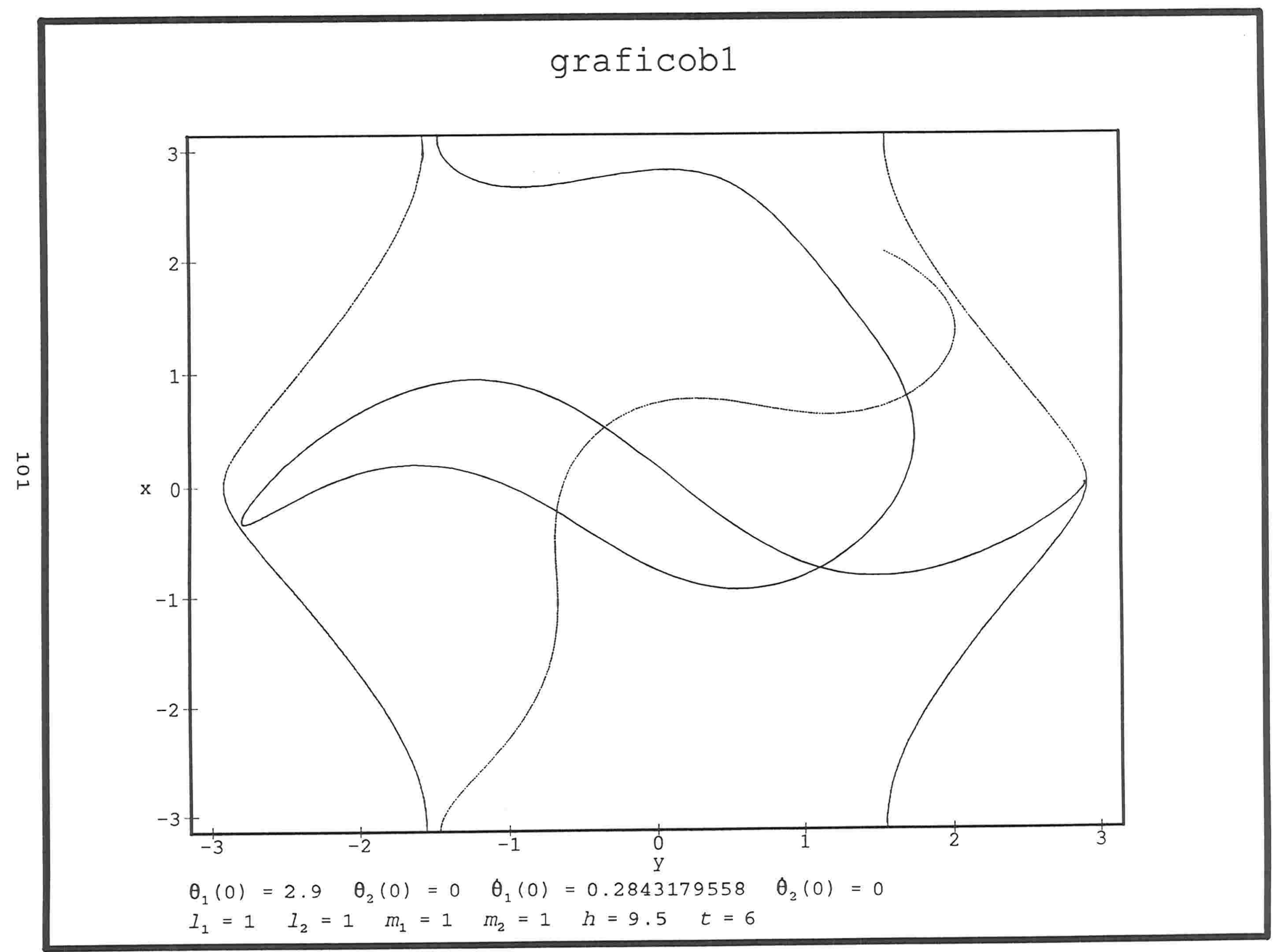




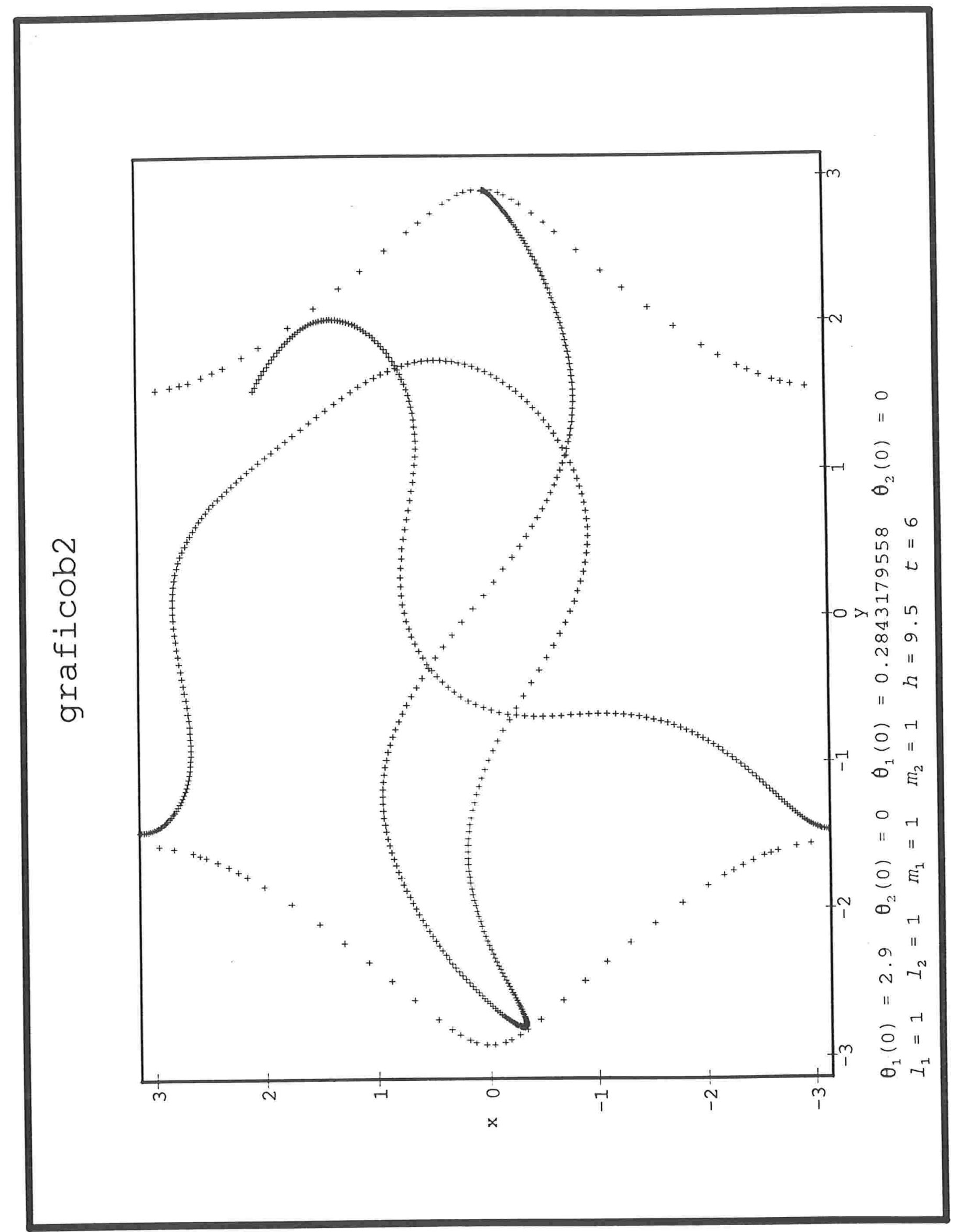




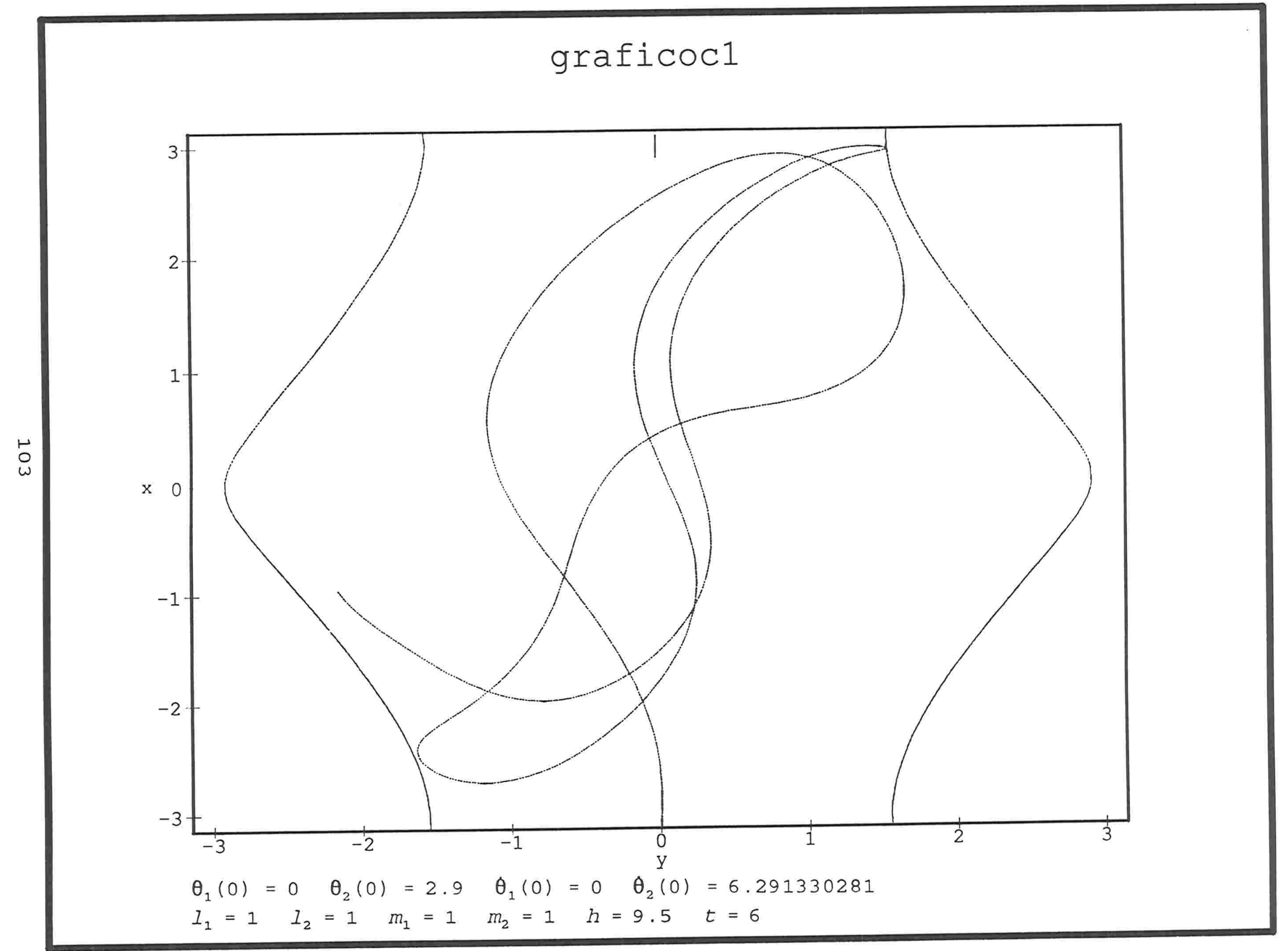


graficoc2

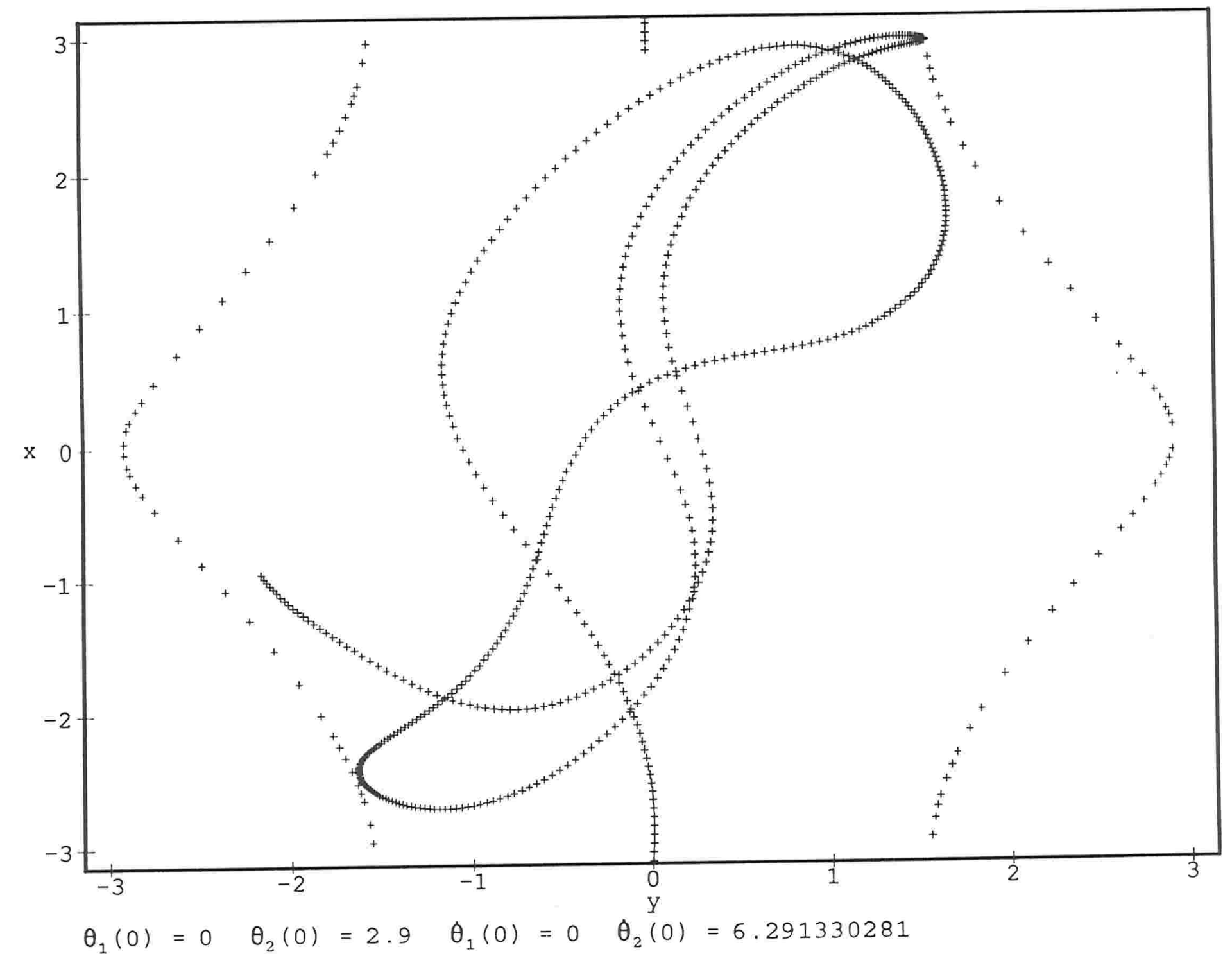

$$
\begin{aligned}
& \theta_{1}(0)=0 \quad \theta_{2}(0)=2.9 \quad \theta_{1}(0)=0 \quad \theta_{2}(0)=6.291330281 \\
& I_{1}=1 \quad I_{2}=1 \quad m_{1}=1 \quad m_{2}=1 \quad h=9.5 \quad t=6
\end{aligned}
$$




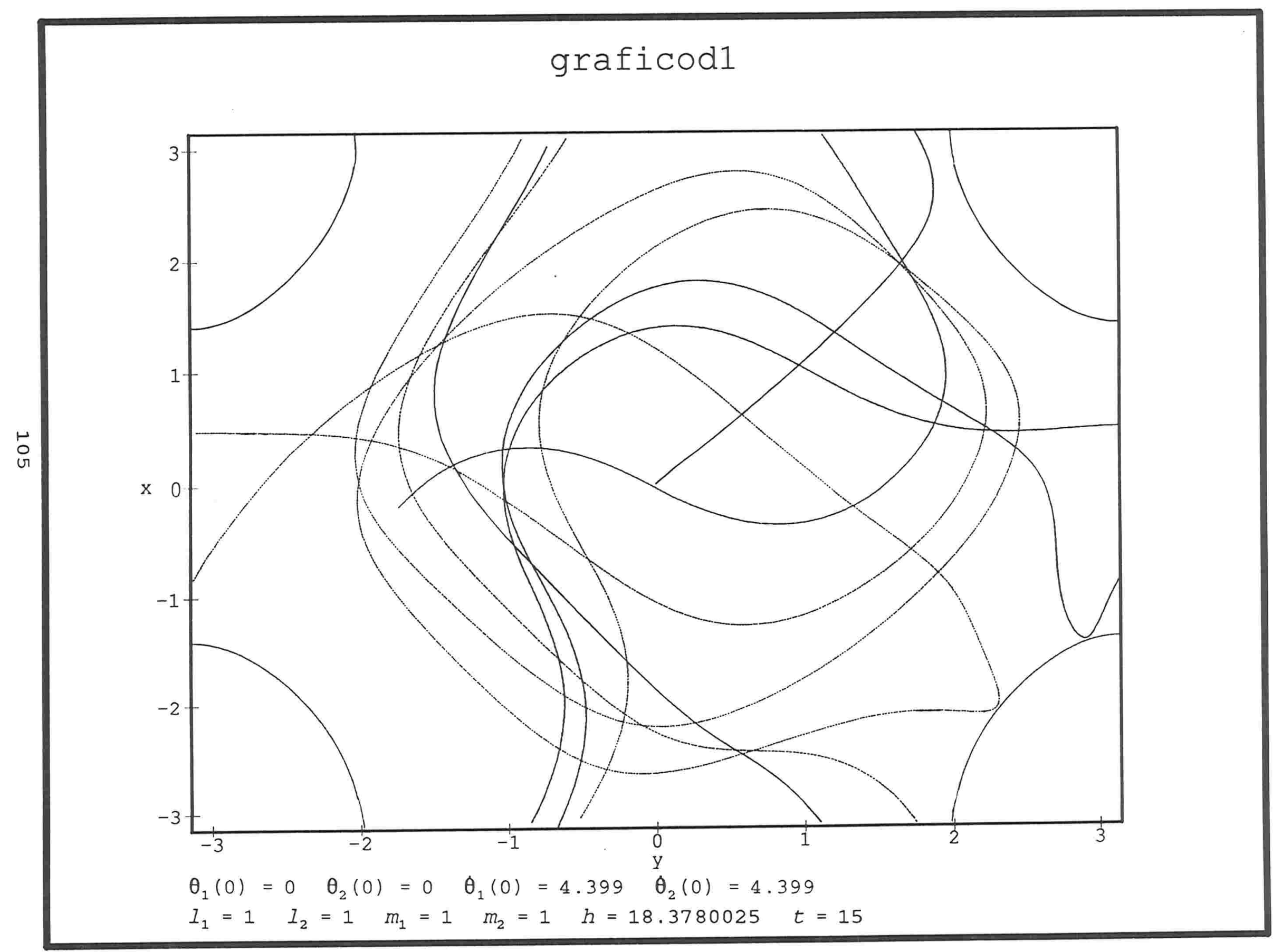




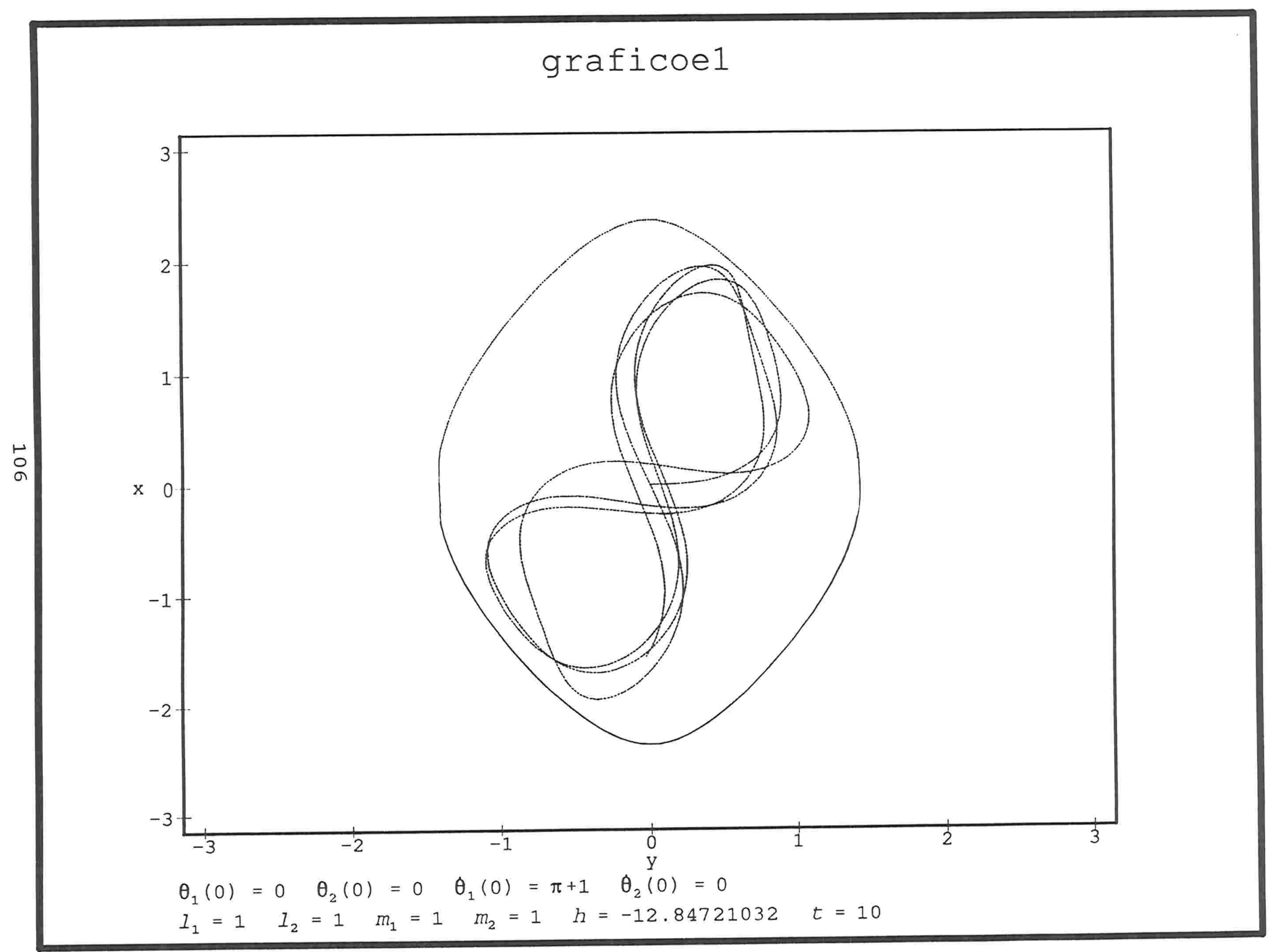




$$
8
$$


<smiles>C1CC2CC12</smiles> 


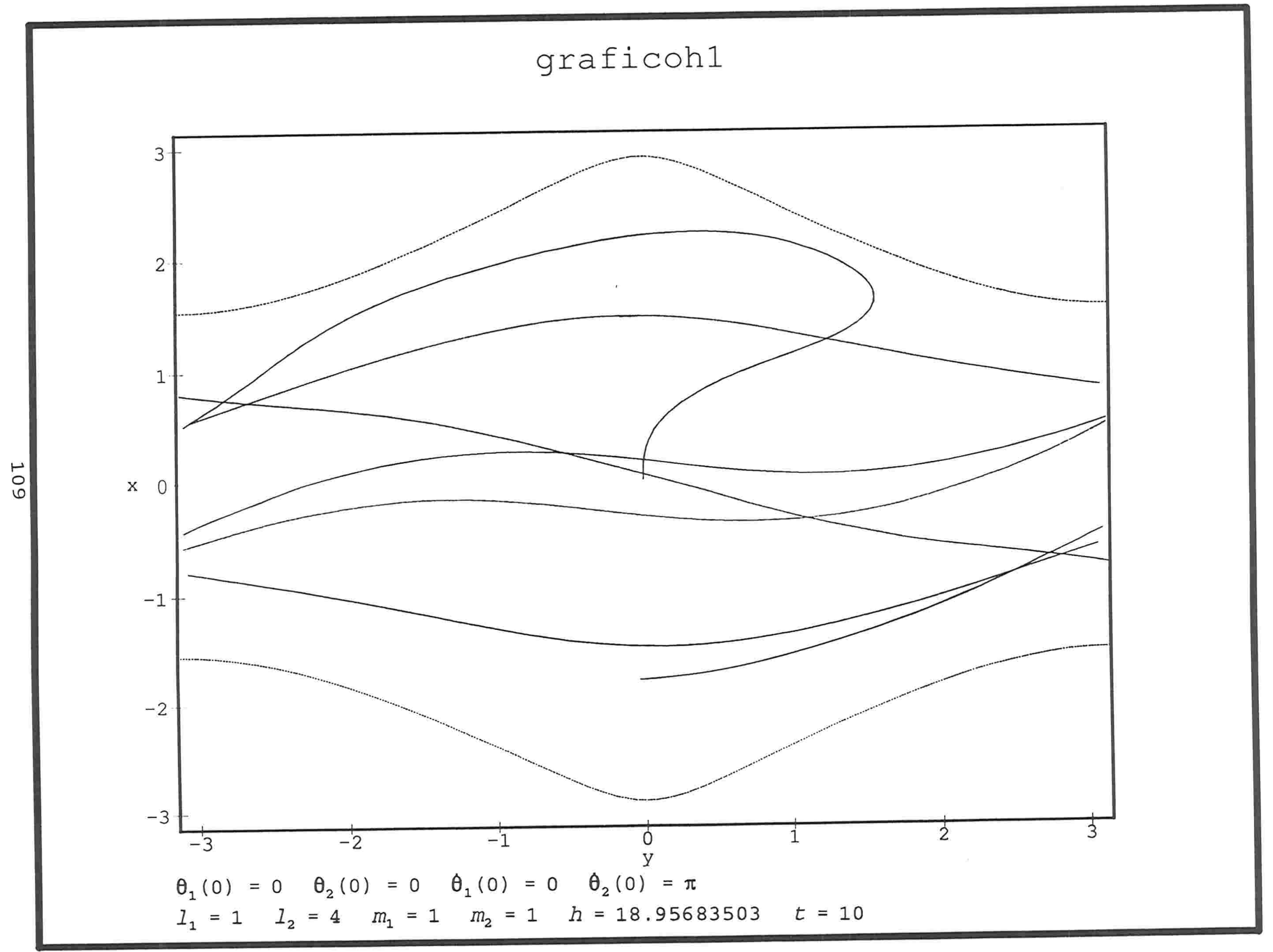




\section{graficoil}

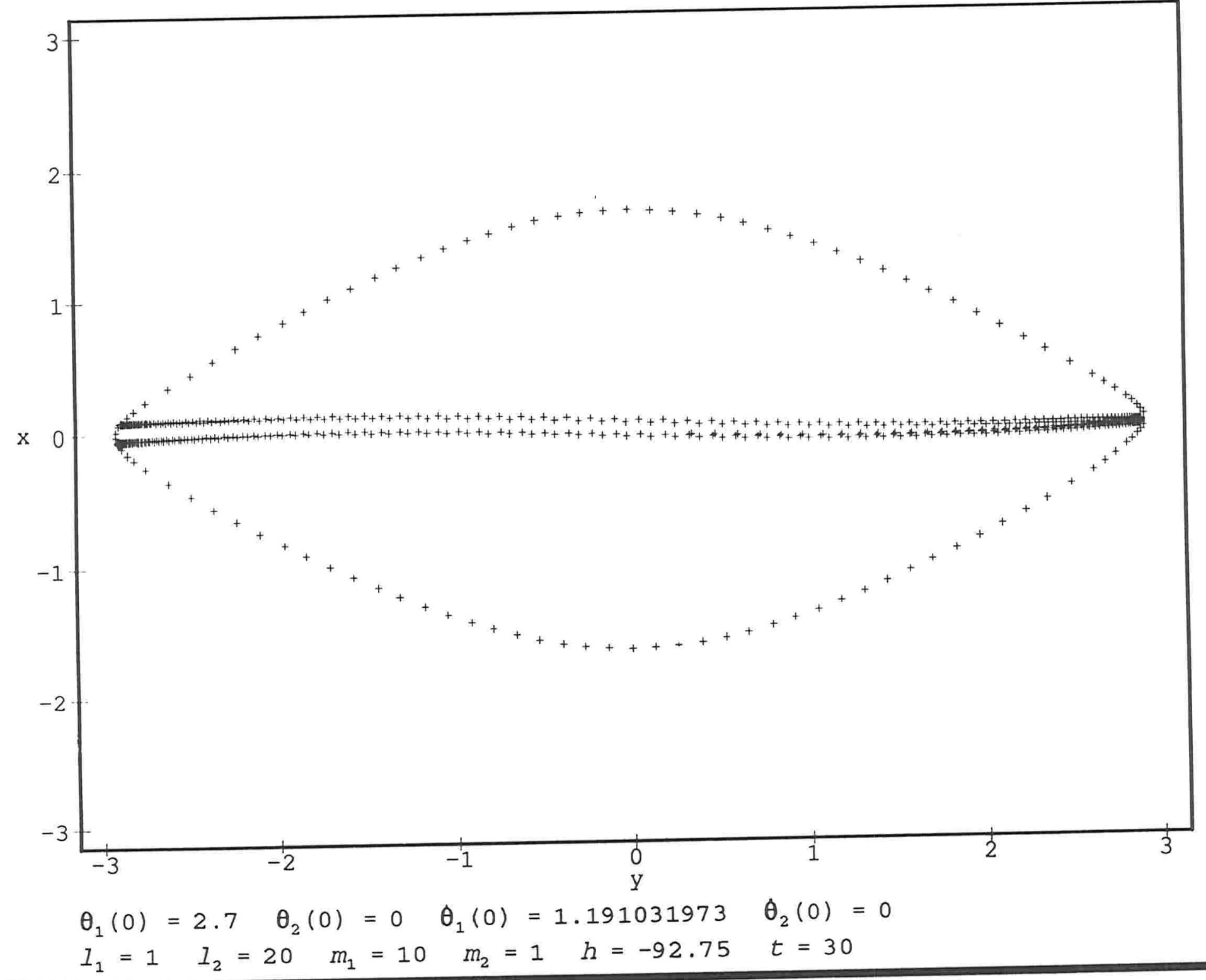




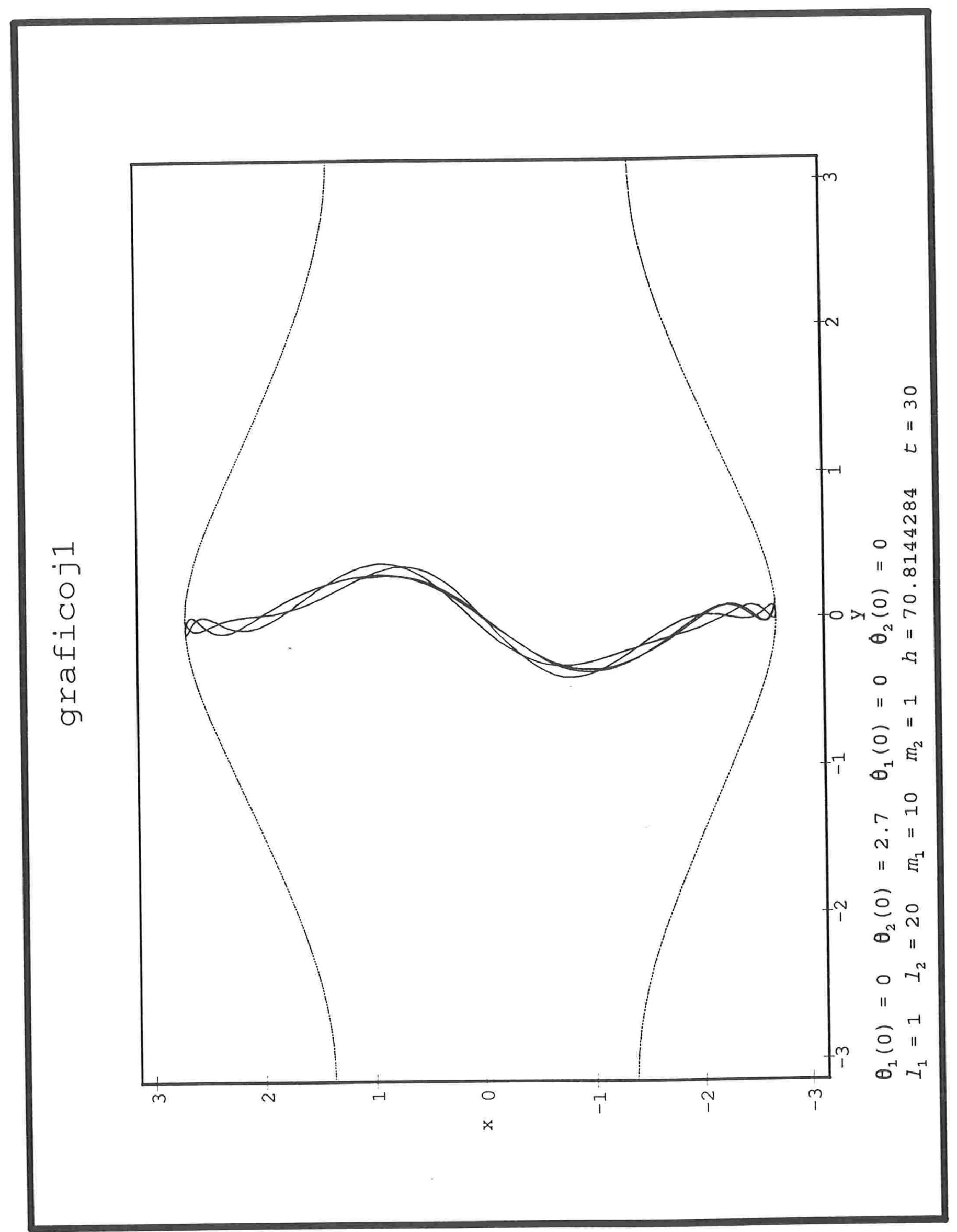




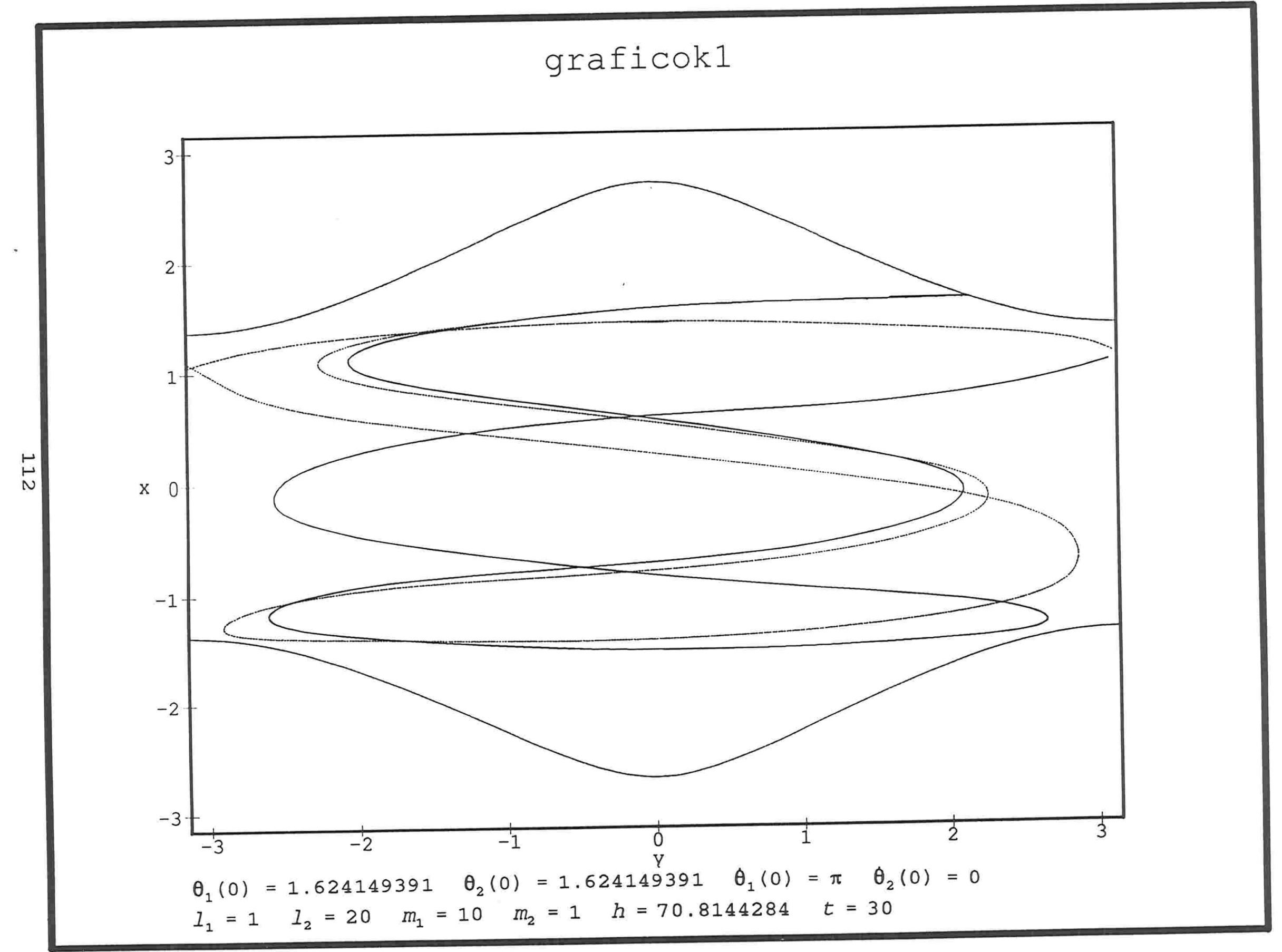




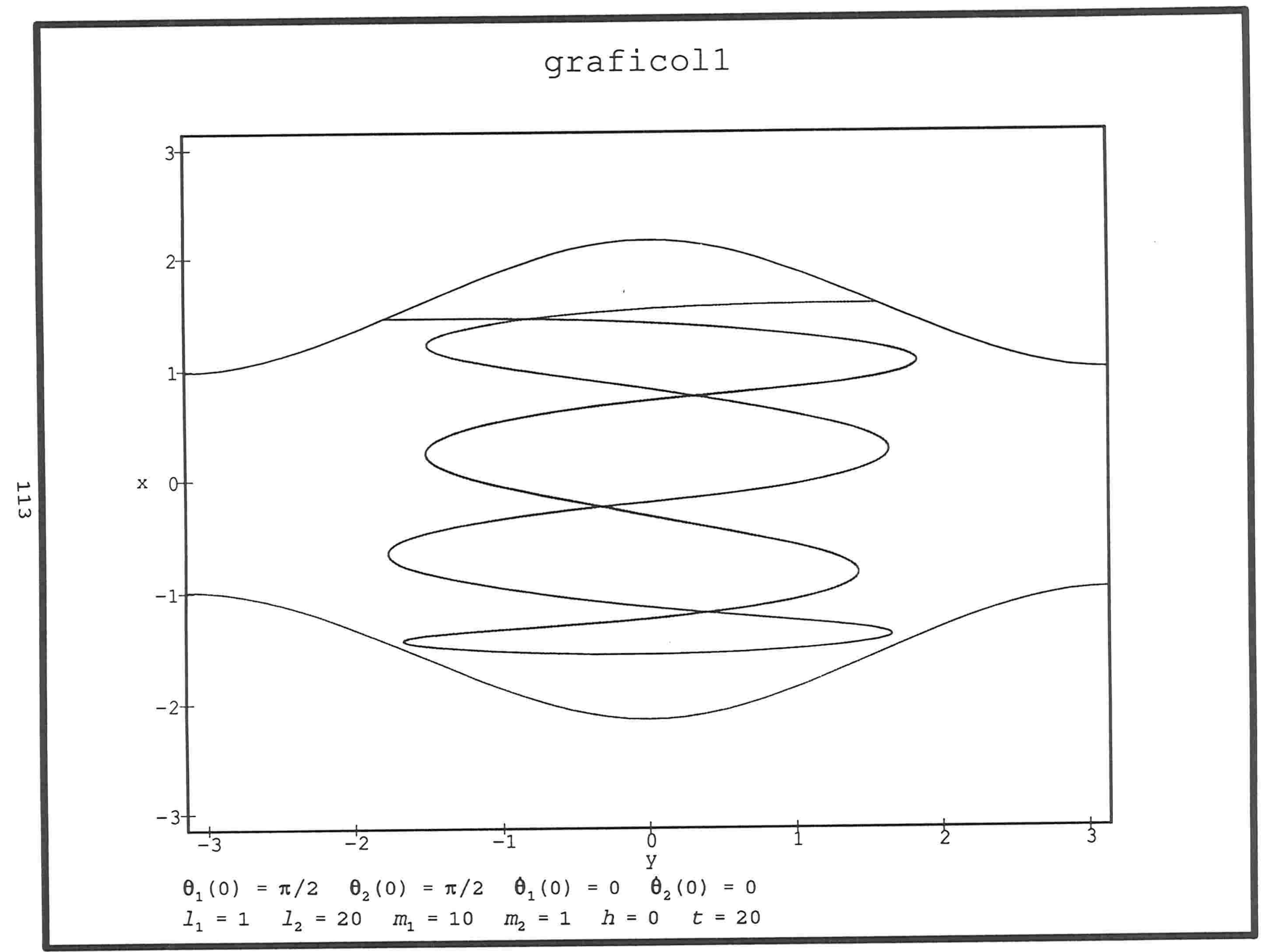


graficol2

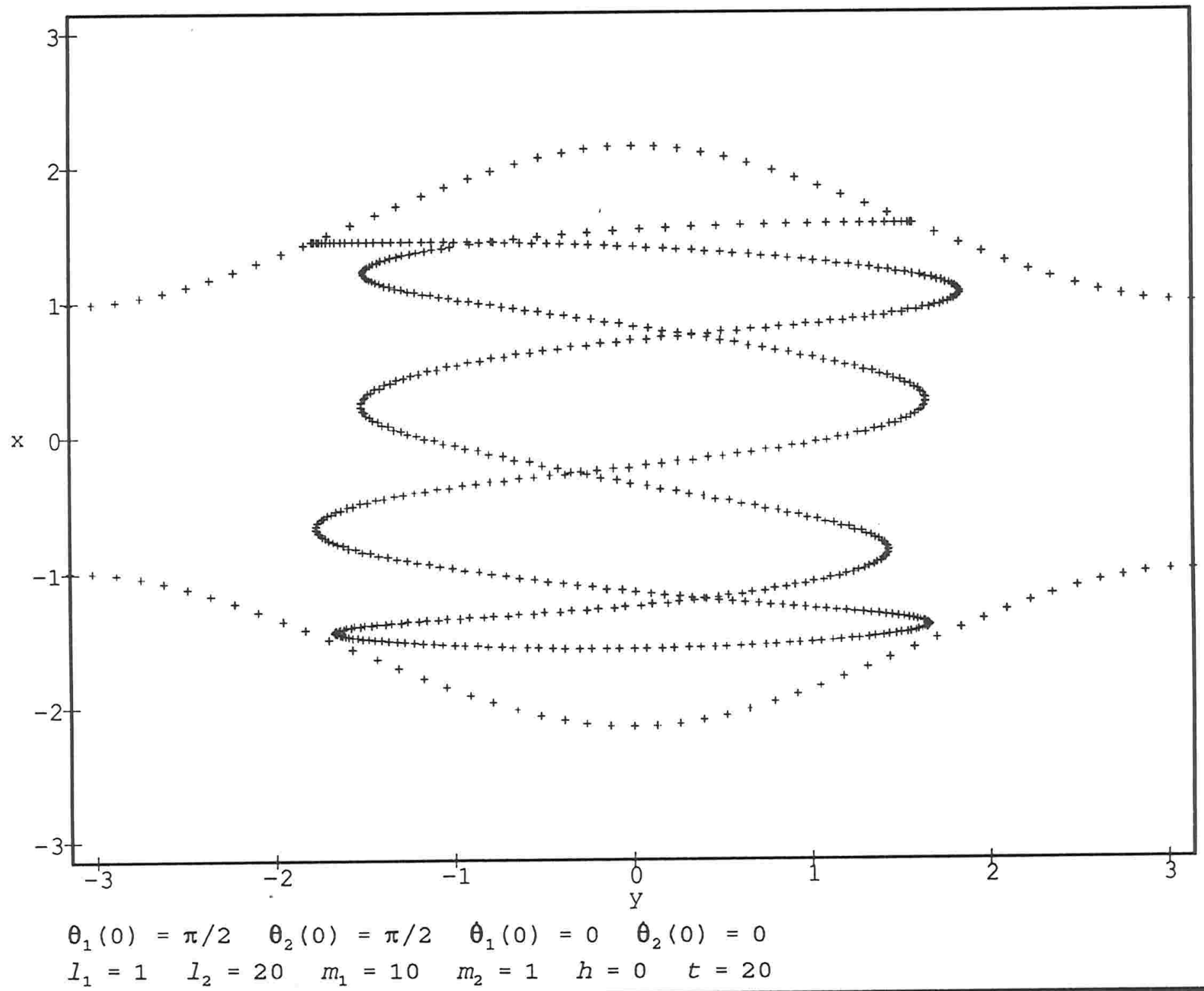


<smiles>C1=CC=C1</smiles> 


\section{APÊNDICE}

/*

Método de Runge-Kutta de $4^{\mathrm{a}}$ Ordem

Entrada de Dados

*1

\#include $<$ stdio. $\mathrm{h}>$

\#include < math.h $>$

\#define N 30000

int $\mathrm{n}$;

/*

Dimensionamento dos vetores

*/

double $t[N], x[N], y[N], w[N], z[N]$;

/*

Funções:

*1

double fnfl (t, x, y, w, z)

double $t, x, y, w, z$;

\{

return(w);

\}

double fnf2 (t, x, y, w, z)

double $t, x, y, w, z$;

\{

retun(z);

\}

double fnf3 (t, x, y, w, z)

double $t, x, y, w, z$;

\{

return $\left.\left(w^{*} w^{*} \sin (x-y) * \cos (x-y)+4 * z * z * \sin (x-y)-10 * \sin (y) * \cos (x-y)+20 * \sin (x)\right) /(-2+\cos (x-y) * \cos (x-y))\right)$;

\}

double fnf4 $(t, x, y, w, z)$

double $\mathrm{t}, \mathrm{x}, \mathrm{y}, \mathrm{w}, \mathrm{z}$;

\{

retur( $\left.\left(2 * z * z * \sin (x-y) * \cos (x-y)+w^{*} w * \sin (x-y)-10 * \sin (y)+10 * \cos (x-y) * \sin (x)\right) /(-2+\cos (x-y) * \cos (x-y))\right)$

\}

void main(argc, argv)

int argc ; char *argv[];

\{

int $\mathrm{i}, \mathrm{j}, \mathrm{f}$, delta, $\mathrm{c}=0$, af;

double

dt,kx1,ky1,kw1,kz1,kx2,ky2,kw2,kz2,kx3,ky3,kw3, kz3, kx4, ky4,kw4,kz4; 


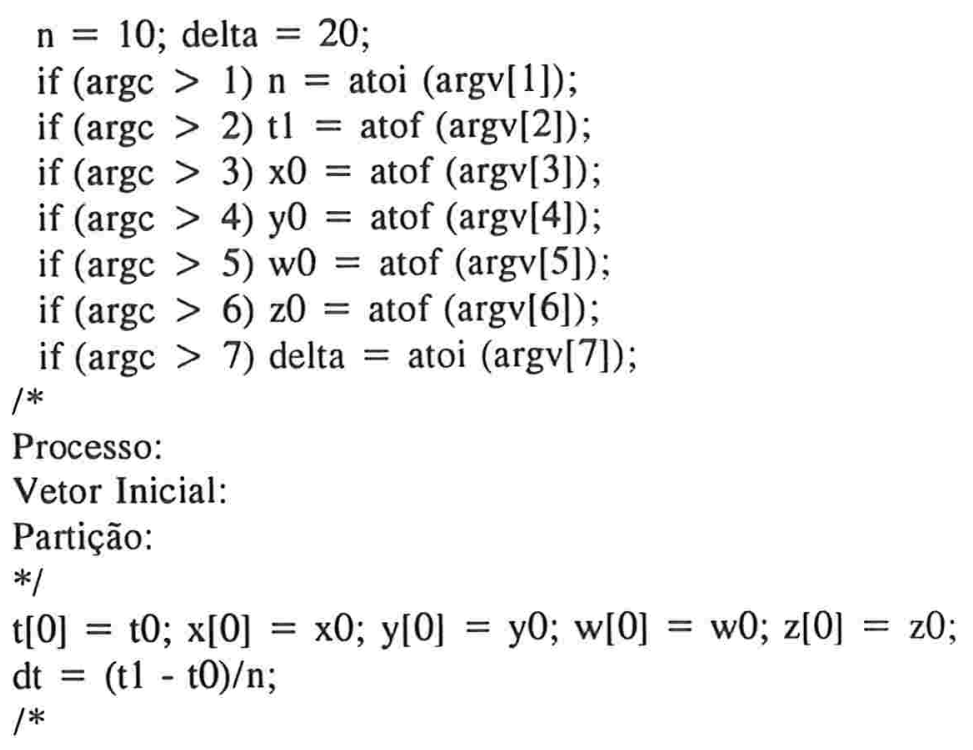




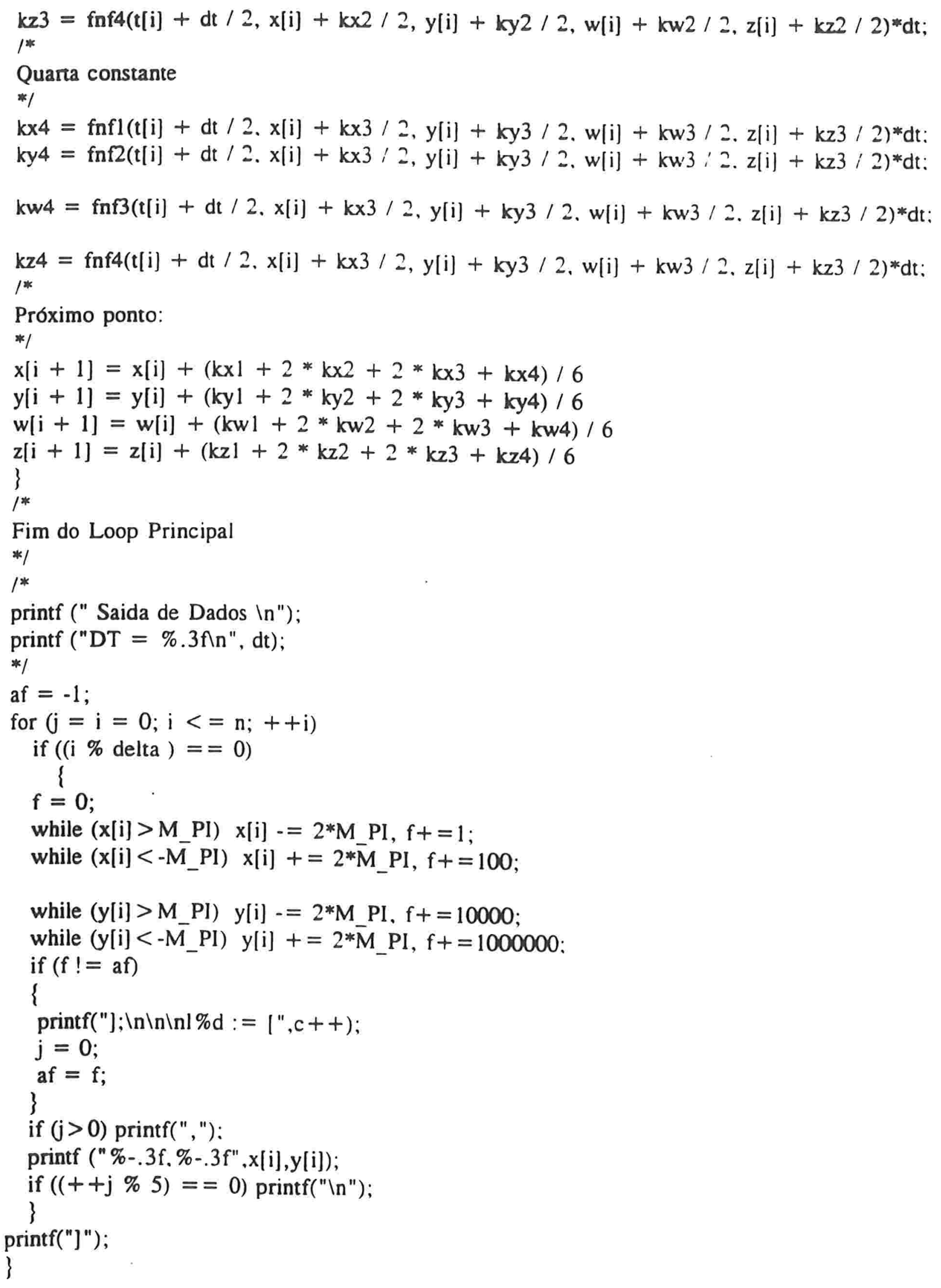




\section{REFERÊNCIAS BIBLIOGRÁFICAS}

01) CARMO, M. P. Geometria Riemanniana. IMPA-CNPq, Rio de Janeiro, 1979. 238p. (Projeto Euclides).

02) HELGASON, S. Differential Geometry, Lie Groups and Symmetric Spaces. New York, Academic Press, 1978. 628p. (Pure and Applied Mathematics, 80).

03) KOBAYASHY, S.; NOMIZU, K. Foundations of Differential Geometry. New York, Interscience, University of California, Berkeley, 1963. v.1 (interscience Tracts in Pure and Applied Mathematics, 15).

04) KULKARNI, R. S. Curvature Structures and Conformal Transformations. J. Differential Geometry, vol.4, n.4, p.425, dec., 1970.

05) LAUGWITZ, D. Differential and Riemannian Geometry. New York, Academic Press, 1965. 238p.

06) MERCURI, F.; RIGAS, A. Curvatura e Topologia. Fortaleza, UFCE, 1978. 120p. (Escola de Geometria Diferencial).

07) MILNOR, J. W. Topology From the Differentiable Viewpoint. Charlottesville, University Press of Virginia c1965. 64p.

08) PIN, O. C. Curvature and Mechanics. Advances in Matematics, vol.15, n.3, p.269-311, mar., 1975,

09) PIN, O. C. A Geometrical Approach to Classical Mechanics. In: CESARI, L.; HALE, J. K.; LASALlE, J.P., eds. Dynamical Systems: Proceedings of the International Symposium held at Brown University, Providence, 1974. New York Academic Press, 1976. v.1. p.41-46.

10) POLLARD, H. Mathematical Introduction to Celestial Mechanics. Englewood Cliffs, Prentice-Hall, c1966. 
12) QUAN, P. M. Riemann Geometry and Mechanics: The Kepler problem. In: AVEZ, A.; BLAQUEIRE, A,; MARZOLLO, A.; eds. Dynamical

Systems and Microphysics: Geometry and Mechanics. New York, Academic Press, 1982. p.91-108.

13) SMALE, S. Topology and Mechanics I. Inventiones Mathematicae. vol.10, p.305 - 331, 1970.

14) SMALE, S. Topology and Mechanics II. The Planar n-Body Problem. Inventiones Mathematicae, vol.11, p.45 - 64, 1970.

15) WOLF, J. A. Spaces of Constant Curvature. New York, McGraw-Hill, c1967. 408p.

16) YANO, K. Integral Formulas In Riemannian Geometry, New York, Tokyo Institute of Technology, Marcel Decker, 1970. 156p. (Pure and Applied Mathematics, 01). 